\title{
Agent-based modeling for migration and modern slavery research: a systematic review
}

\author{
Alys McAlpine $^{1}$ (D) $\cdot$ Ligia Kiss $^{1,2}$ (D) $\cdot$ Cathy Zimmerman ${ }^{1} \cdot$ Zaid Chalabi $^{3,4}$
}

Received: 27 March 2020 / Accepted: 16 July 2020 / Published online: 18 August 2020

(c) The Author(s) 2020

\begin{abstract}
This systematic review aims to synthesize how agent-based modeling (ABM) has been used in migration and modern slavery research and provide the basis to model development for social science researchers exploring the use of ABM. We searched five bibliographic databases using two terminology categories: (1) migration or modern slavery terminology; (2) complex system methods terminology. Two reviewers conducted independent article screening. Peer-reviewed articles presenting original migration or modern slavery ABMs were included. Data extraction included model development steps and model characteristics. The dataset was synthesized and compared across studies. We identified 28 articles for inclusion. Many of the ABMs tested theories and about half were based on empirical data. Model development varied considerably and reported methods were extremely opaque. Only five studies used a structured development framework. The most common model involved agents deciding whether and where to migrate and attempting migration. Climate change was a common exogenous scenario modeled. Most of the ABMs did not undergo any sensitivity analysis or validation.ABM has a greater capacity to account for heterogeneous and dynamic decision-making than more frequently applied methods in research on migration and modern slavery. However, there is still a paucity of studies adopting ABM methods. These reviewed ABMs highlight gaps in the reporting and implementing of model development. ABM is a promising technique to address many urgent and complex questions in research on migration and modern slavery to better support decision-makers, but addressing current methodological gaps is a critical first step.
\end{abstract}

Keywords Agent-based modeling · Complex systems · Migration · Modern slavery · Trafficking

Electronic supplementary material The online version of this article (https://doi.org/10.1007/s4200 1-020-00076-7) contains supplementary material, which is available to authorized users.

Alys McAlpine

alys.mcalpine@1shtm.ac.uk

Extended author information available on the last page of the article 


\section{Introduction}

This review aims to explore how agent-based modeling (ABM), a complex systems science method, has been applied to research on migration or modern slavery, and to synthesize the ABM model development in this field of study to inform future applications of these methods. ABM, particularly in relation to socio-ecological systems modeling, has increasingly been employed to study many dynamic multi-scale research questions, such as ecosystem management, collective resource sharing and a particularly well-established method in studies of land use [1]. Ecological complex systems modeling has paved the way for bridging many other disciplines, including social sciences, with complexity science. We believe that interdisciplinary ABM methods present an opportunity to address many critical, unanswered, and complex questions in migration and modern slavery research and at the nexus of these two research topics. In a first step to advance the adoption of these methods in this field of research, this review synthesizes and assesses the use of ABM in migration and modern slavery research to date.

This review builds on the work of modelers that have been using and reviewing the use of $\mathrm{ABM}$ to explore emergent trends in migration, particularly migration influenced by environmental changes, social networks and decision processes, such as utility maximization [2-4]. Bell, Hernandez and Oppenheimer pointed out that many of the ABMs in this field focus on singular push-factors (e.g. climate change) and usually only consider the pull-factors or destination choices after the agents have surpassed a push-factor threshold [3]. Klabunde and Willekens explored the decision-model choices for migration ABMs that span several dimensions (forming expectations, evaluating options, the complexity of the decision, networks influencing decisions, etc.) and it is clear that not all of these dimensions are critically addressed or at least not articulated in current migration ABMs [2]. Many ABM modelers have asserted that without clear documentation of the full spectrum of model development (or 'modeling cycles', including final model validation), there are limitations to the contributions ABMs can claim or methodological advancements they can foster $[1,5]$. In this vein, our aim is to contribute a systematic review of the model development of migration ABMs to allow for more informed dialogue on the gaps in model development and reporting of model development.

\section{Why explore ABM for research on migration and modern slavery?}

Many academics and practitioners are trying to understand the complexities of human migration to inform policy and practice, particularly to promote safer labor migration and address issues of modern slavery. For example, the United Nations' Sustainable Development Goals include taking "immediate and effective measures to eradicate forced labour, end modern slavery and human trafficking" (Goal 8.7) and to "facilitate orderly, safe, regular and responsible migration and mobility of people" (Goal 10.7) [6]. There is a growing body of research focusing on the causal pathways between low-wage labour migration and forms of labour exploitation, also known as modern slavery [7, 8]. Yet, there is limited evidence to explain the nexus 
between low-wage labour migration and modern slavery completely [9], including evidence gaps on the complex mechanisms that contribute to entry into slavery-like conditions versus decent work $[8,10,11]$. That is, we have yet to understand the pathways that lead to different migration-related outcomes, in addition to understanding the drivers of migration as some of the previous ABM migration models have explored.

As an example, in public health, the disciplinary background of some of this reviews' authors, we have become increasingly aware that while the conventional epidemiological approaches can be useful to capture the effect of exposures on singular outcomes or offer estimates at the aggregate population-level data, they are often not suited to investigate the complex non-linear causal pathways that affect health (e.g. complex health behaviour problems) [12]. For example, epidemiological methods can describe well the aggregate experiences of migrant exploitation and health outcomes, such as the health outcomes of human trafficking [13], but they do not capture the complex system dynamics of labor migration and modern slavery. $\mathrm{ABM}$ has recently been recognized by the public health research community as a complex systems approach that can explore causal complexities inherent to human behavior and population health [14-16].

This paper gives a brief introduction to ABM of social systems and describes current applications of these methods for exploring complex systems in migration and modern slavery research. Finally, this paper presents findings to contribute methodological learning that is transferable across many social and health science disciplines.

\section{Agent-based modeling for complex social systems}

Agent-based modeling is a method for studying complex systems. A complex system contains many parts interacting at the individual (micro) level in an irregular way and generally producing non-linear outcomes with regularity at the systems, population or aggregate (macro) level [17-19]. Stephen Wolfram, one of the founding scholars on complex systems theory, said, "It is now a crucial problem for many areas of science to elucidate the mathematical mechanisms by which large numbers of such simple components, acting together, can produce behaviour of the great complexity observed" [20]. ABM is a computational simulation technique that has gained increasing popularity in recent decades across many social science disciplines to study social complexity in human systems [19]. For example, public health and health systems research has employed ABM methods to study communicable and non-communicable diseases, health behaviors, and other topics in social epidemiology [21]. These methods are increasingly recommended to evaluate complex health systems and prevention interventions [15, 22].

In simple terms, an ABM consists of a simulated population of autonomous, goaloriented individuals (agents) that are capable of interacting with each other (agentto-agent) or with their simulated environment (agent-to-environment). Agents can be assigned specific attributes (demographics, attitudes, risk aversion, etc.) that in combination with the rules of the model (how individuals behave, make decisions, 
interact with their surrounding environment, etc.) govern the steps that result in the dynamic and emergent outcomes [23].

Experts from both computer science and social science have written extensively on the unique capabilities and usefulness of ABMs in social science research [17, $19,24,25]$. In short, ABMs have a bottom-up approach that enables exploration of individual-level behaviors and causal mechanisms that lead to macro-level aggregate emergent phenomena, which can only be inferred from modeling the individuallevel interactions. The task is to replicate a population, the environment and its patterns in such a way that the model produces similar emergent properties or outcomes to the observed outcomes in the real world [26]. Some scholars in this methodological field argue that the next frontier of explanatory social science research is being able to 'grow' the phenomenon of interest from micro-level rules [17]. For example, recent $\mathrm{ABM}$ research on health-pertinent behaviors, such as smoking, diet and interpersonal violence, has successfully simulated groups of individuals that move in a social or physical space, exchange information, model behavior, copy behavior and make decisions [21, 27]. All of these individual actions in aggregate replicate realworld trends in health behaviours and outcomes, such as smoking habits or obesity, amongst close ties in social networks.

\section{An example of a complex dynamic system in migration research: Low-wage labour migration and exploitation}

Globally, the majority of migrants participate in the labor force [11]. The majority of victims of modern slavery are low- or no-wage laborers and a significant proportion are migrant workers [10]. These two global phenomena, low-wage labor migration and modern slavery, are linked in complex and dynamic ways. Figure 1 is a conceptual framework visualisation depicting some of this complexity. In Fig. 1, we present the migrant on a pathway between origin and destination and, in some cases, a pathway that returns to origin. Individuals very rarely migrate without the help of social or intermediary networks, such as migrant networks at destination or labor recruitment networks [28]. Thus, we have also presented the migrant connected to a
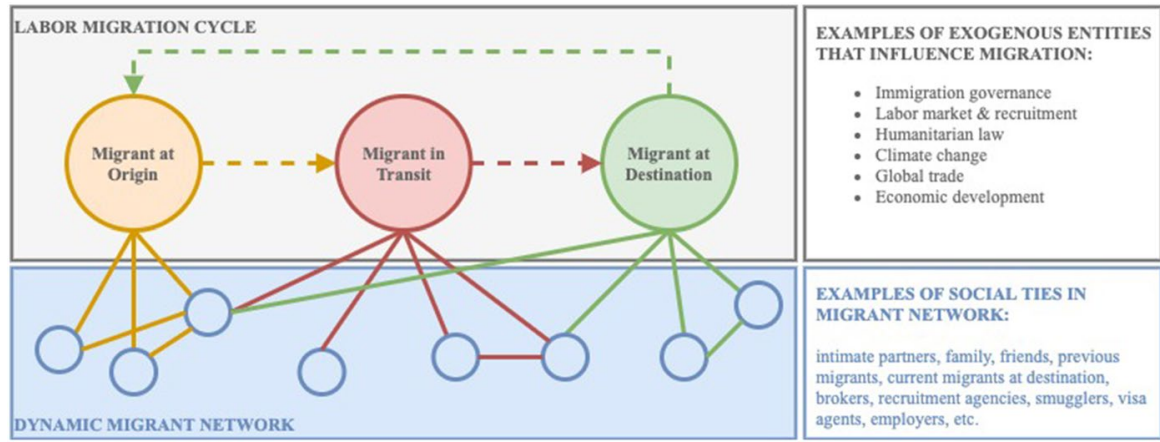

Fig. 1 Framing low-wage labor migration as a complex dynamic system across multiple geographic spaces: Agents, networks and exogenous influencers 
dynamic network of possible relations that evolves throughout the migration cycle. These migration-facilitation networks and any associated social or migratory norms are also influenced by exogenous entities, such as climate change, economic development and labor recruitment [29]. An individual experience of migration is influenced by all of these factors (for example, individual behaviors, social networks, social norms, immigration governance or labour markets). Not included in Fig. 1 are the range of outcomes at every stage in the migration cycle on individual and population levels that operate through feedback mechanisms which, over time, cause emergence of population-level migration behaviour which can only be inferred from individual-level interactions.

Theoretical developments in the fields of migration research and public health research draw attention to the multi-causal, dynamic, multi-directional and non-linear nature of complex social problems, including the ecological systems approach to addressing social phenomena and the importance of concepts of social networks, decision-making under uncertainty and bounded rationality $[2,9,16,27,30,31]$. There is a convincing argument for adopting new methods of data collection and analysis that reflect these theoretical concepts. Agent-based modeling enables complex dynamic system simulation that encompasses multiple ecological levels (individuals, households, social networks, migration corridors) and incorporates heterogeneous individual decision-making and behaviors (such as the system in Fig. 1).

Donors, practitioners and policymakers advocating for the protection and wellbeing of low-wage labor migrant workers rely on research to provide evidence on the challenges these individuals face during their migration and employment, to inform effective interventions. To date, a majority of this research has been conducted using conventional statistical or qualitative research methods. The statistical methods implicitly treat labor migration as a one-dimensional, one-directional, linear and static "exposure-response"-type relationship between drivers of migration and migration outcomes. Such methods do not take into consideration the complexity of labor migration or its dynamic nature. These analyses do not account for the various feedback mechanisms governing the interaction of different actors with other actors and with their environment, the time delays between a stimulus (action) and the corresponding response, and the non-linear nature of responses to stimuli. The current body of evidence primarily tries to offer insights into the drivers of migration, the demographics of migration, migration corridors and outcomes, including growing evidence on the range of harmful outcomes, such as unfair recruitment, labor exploitation and forms of modern slavery. But, these analyses do not improve our knowledge on labour migration processes or the nature of the complex exposures and mechanisms related to migration. Every story of migration encompasses information-seeking, decision-making, interactions and exchanges with individuals, groups and systems.

\section{Examples of other ABM reviews}

As referenced earlier, to our knowledge, there are three reviews of migration ABMs relevant to the aim of this review, including Klabunde and Willeken's review focusing 
on decision-model choice including integration of social networks $(n=22$ included publications) [2], Thober and colleagues' review of ABMs of environmental-migration linkages $(n=21)$ [4], and Bell and colleagues' brief background review on ABMs exploring migration push-pull factors to present their migration ABM framework (nonsystematic, $n=\sim 8$ ) [3]. Other examples of ABM reviews include previous reviews on the use of ABM to study other social or health science topics, such as urban crime $(n=45)$ [32], non-communicable diseases (NCDs) $(n=22)$ [33], obesity $(n=38)$ [34], public health (non-systematic, $n=\sim 45)$ [21], and health systems $(n=11)$ [35]. These reviews reported notable increased interest in and application of ABM methods in their respective field of study, despite still small yields compared to other reviews including different methods. The reviews focused on urban crime, NCDs, obesity, and public health all discussed the current gaps in standardised ABM methods and a lack of transparency in model development as a major limitation in the field for model replication and systematic comparison. Cassidy et. al.'s review on the use of ABM and System Dynamics (SD) models in healthcare systems research did not conduct extensive extraction on model development processes, and so did not address these issues, but did conclude that the topic of data source choices is a critical next area of review in complex healthcare systems modeling [35]. The most relevant of all these reviews to the present one is Klabunde and Willekens's review of ABMs of migration, which differs from this review as it focused primarily on the behavioural theories that informed the decision-making models [2]. The present review draws on this previous work and proposes a wider scope of investigation. The key contribution of this review is to incorporate a broader set of search terms to include modern slavery phenomena as well as migration. Furthermore, this review extracted data points on the full spectrum of the $\mathrm{ABM}$ development process, not just the decision-making element. We will reference Klabunde and Willekens's review throughout the methods and discussion sections of this paper, particularly because of the strength of that review's synthesis and analysis of the decision-making process which was not within the scope of this review, but an important topic for ABM development.

\section{Review aim}

This review aims to describe how ABM has been used in research on migration and/or forms of modern slavery. Findings are intended to inform future studies on the application of this methodological approach, including guidance on model calibration, model development, sensitivity analysis, and model validation. This review aims to contribute a summary and assessment of the state of the art of ABM used in migration and modern slavery studies to encourage and inform future adoption of ABM methods in this field. 


\section{Methods}

This review adhered to the Preferred Reporting Items for Systematic Reviews and Meta-Analysis (PRISMA) statement [36]. The full protocol for this review, including comprehensive database search scripts, can be shared upon request to the corresponding author. A condensed version of the search protocol can be found in the Supplementary Materials (SM-1). The summary of the methodology is outlined here.

\section{Sources}

The search was conducted on 5 bibliographic databases covering public health, social science, and computer science disciplines (See Table 1 for details).

\section{Search strategy}

The search strategy included two subject areas: (1) migration or modern slavery; and (2) computational dynamic systems modeling or network analysis methods. We developed a conceptual framework that depicts the review concepts (See Fig. 2) to guide our terminology inclusion in the search scripts (See Table 2). The search scripts are included in the Supplementary Material (SM-1).

\section{Eligibility criteria}

The search only included peer-reviewed articles published between 1 January 1999 and the date of search (See Table 1). Additionally, the full article had to be available in English. We did not apply any exclusion criteria based on the discipline, data sources (quantitative, qualitative, secondary, theoretical, etc.), study type (cross-sectional, longitudinal, trial, etc.), quality of the study, or the publishing journal. The studies needed to meet two criteria pertaining to (1) the study topic and (2) the study methodology.

\section{Study topic}

The research questions and outcomes of the study addressed one or more of the following themes: migration flows; drivers of migration; demographics of migrant populations; migrant social networks during migration planning, implementation or job-seeking; migrant labor; immigration policy; labor recruitment; safer labor

Table 1 Bibliographic database searches

\begin{tabular}{lll}
\hline Databases & Date of search & Filters applied \\
\hline Web of Science, Scopus, PubMed & $09 / 06 / 2019$ & Document type: Articles; Reviews \\
MathSci, arXiv & $18 / 09 / 2019$ & Years: 1999-2019 \\
\hline
\end{tabular}




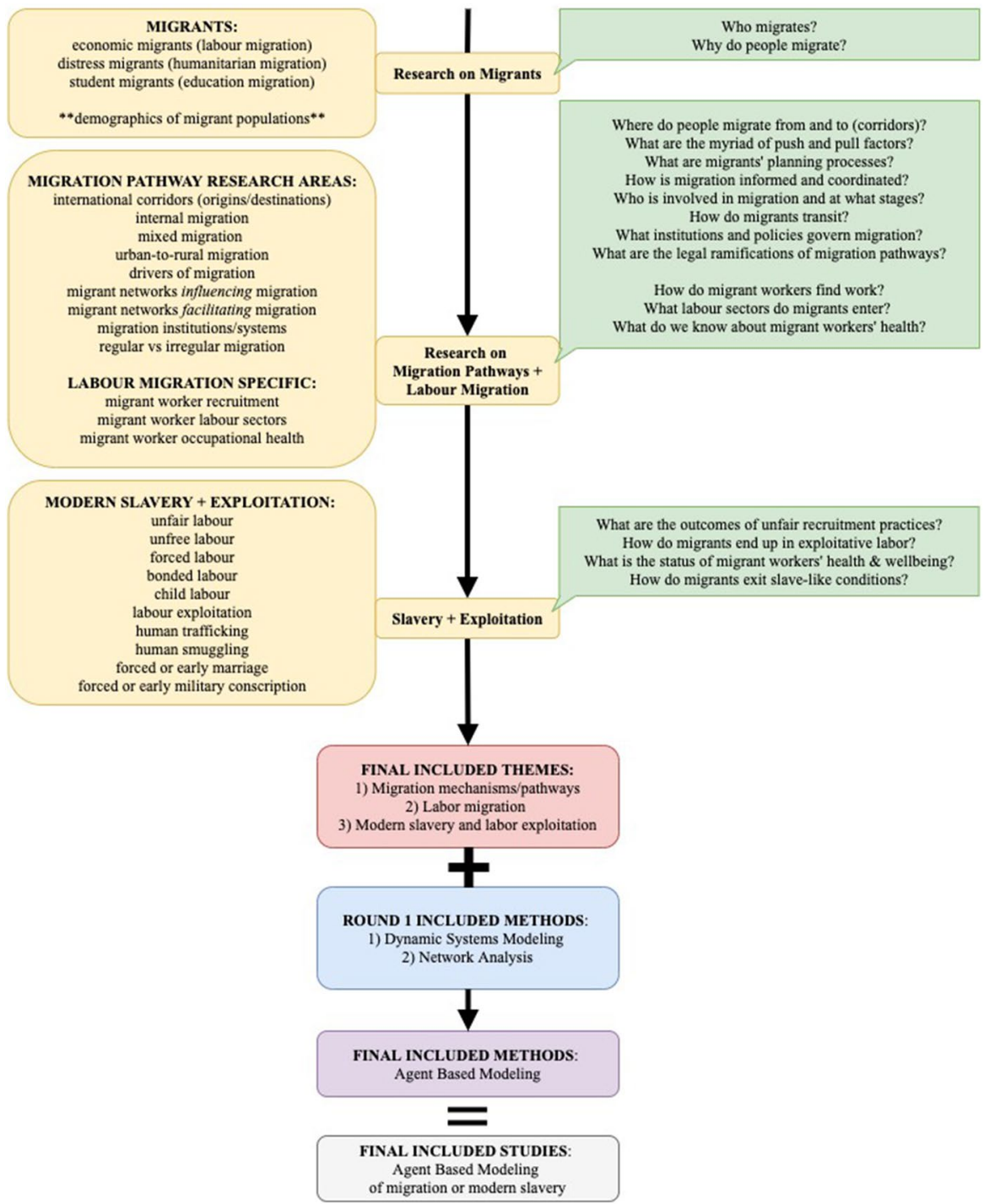

Fig. 2 Conceptual framework of key concepts applied for the review's search

migration interventions or migrant worker health interventions; remittance flow; return migration; modern slavery or child labor (including child soldiers).

The search protocol outlined similar topics that would not be included: disease spread through migration; general migrant population health at destination (unless specific to migrant workers or forms of modern slavery); humanitarian coordination or service delivery broadly; disaster preparedness or short-term emergency evacuation; residential or local migration (for example, urban sprawl, residential neighborhood choice, etc.); ethnic diversity at destination (without exploring 


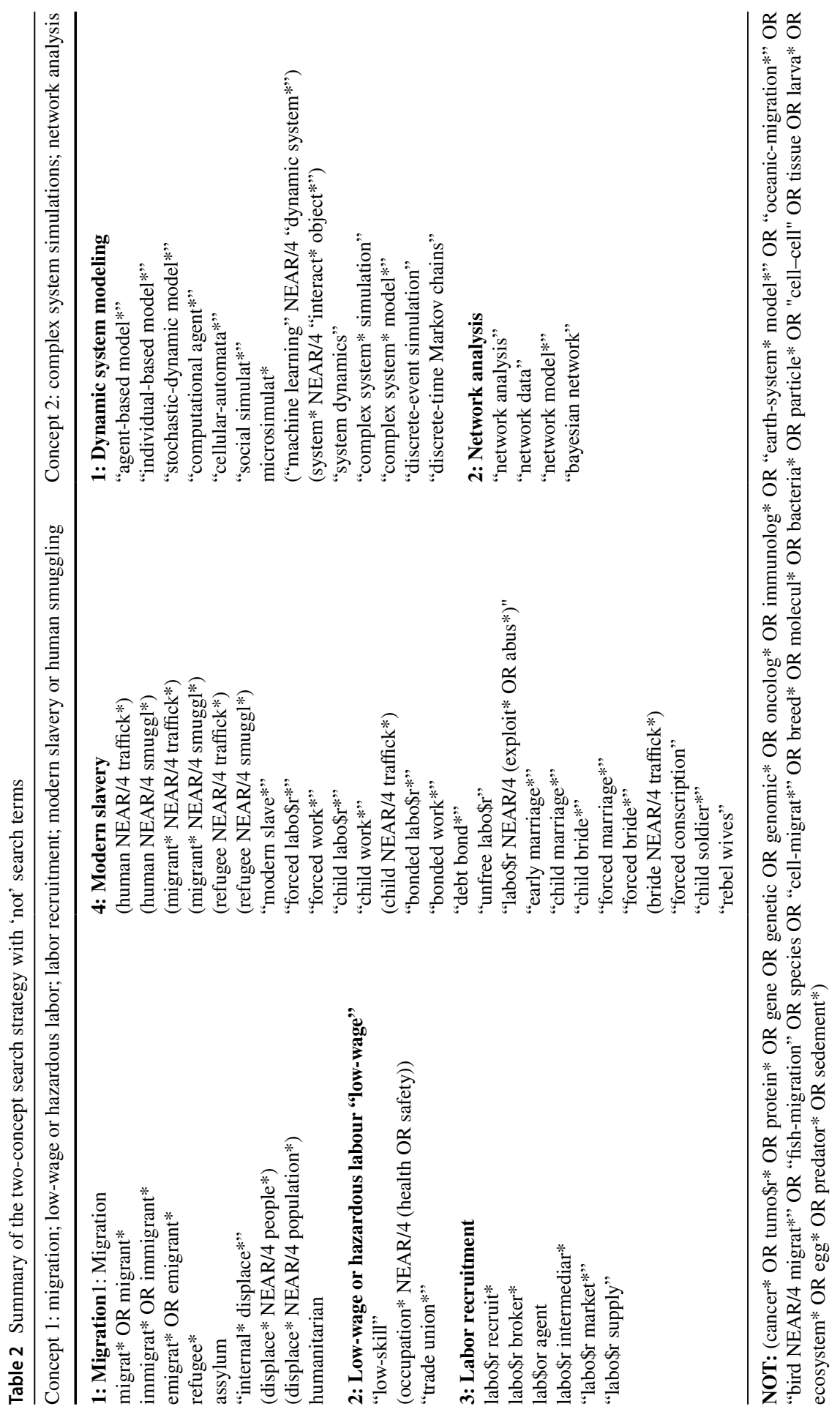


migration mechanisms or networks that facilitated migration); immigrant cultural integration at destination (except where relevant to employment or earnings as described above); animal migration; tourism; or broad low-wage or hazardous occupation research without disaggregation by migrant status.

2. Study methodology

The search had two-stage inclusion criteria. The first stage included a broader range of computational dynamic systems and network methodologies that could provide more comprehensive findings on the types of innovative methodologies being used in migration studies. This stage also was designed to prepare for a paucity of literature on ABMs to review in this subject area. The secondstage inclusion criteria, which was applied to the articles that were eventually fully extracted for this review, included only original research that used ABM. The results described in this paper are based on the articles identified using the second-stage criteria.

\section{Study selection}

The full database yields were uploaded to the Rayyan systematic review software and de-duplicated [37]. Two co-authors completed independent-blind abstract reviews for the 1707 articles. The reviewers met in person to discuss a collection of 100 articles that received conflicting decisions (one reviewer included and one reviewer excluded). The two reviewers were able to agree on inclusion or exclusion for all 100 articles through referral to the study protocol and without the need for a third reviewer. In total, 137 articles were included after the first round of abstract screening. Then a second round of abstract screening was completed to narrow the yield to agent-based models only and to be full-text screened. At the final screening stage, 58 articles were full-text screened, and of these, 28 articles met the final inclusion criteria. For more details on the screening process and reasons for exclusion, see Fig. 3.

\section{Data extraction}

This is a review of the methodology of these studies, not a synthesis of findings. Therefore, the data extraction process captured the authors' affiliations and study aim, model inputs, model development, model process and the analysis and validation methods completed by the authors. This method is similar to systematic reviews of ABMs on other social or health science research topics that focused on the study design, model specifications (agents, environment, decision rules), and model analysis (sensitivity analysis, model validation) for data extraction and synthesis [33, 34, 38-41]. The data extraction tool was informed by the guiding questions outlined in the Overview, Design Concepts and Details + Decision-Making (ODD+D) protocol for ABM development, which is an adaptation of the original Overview, Design 


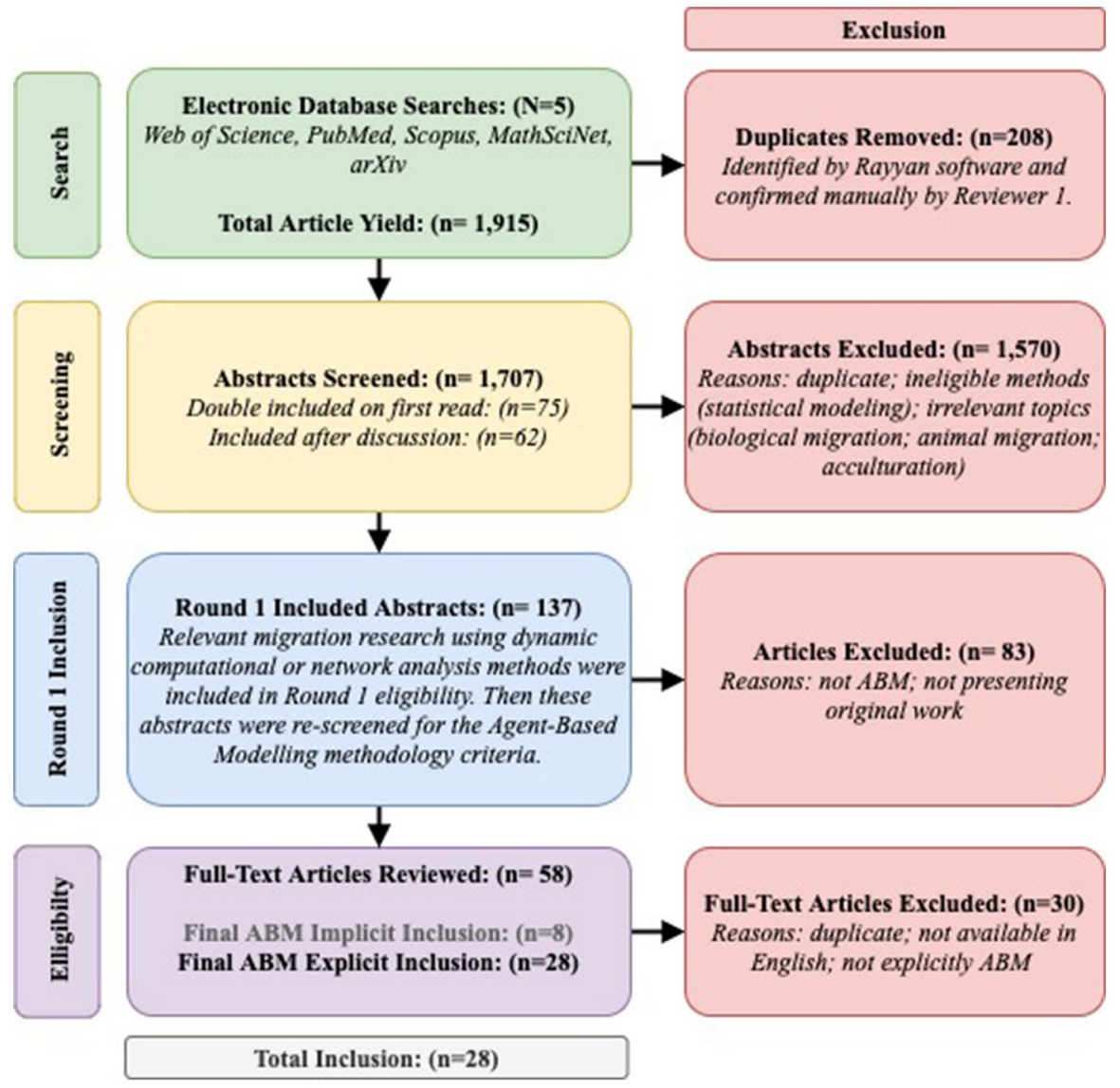

Fig. 3 PRISMA systematic review flowchart

Concepts and Details (ODD) protocol developed to standardize descriptions of individual-based models (IBMs) and ABMs [5, 42, 43]. Before the development of the ODD framework in 2006, computer-simulation models, such as ABMs, did not have standardised guidelines for dissemination [42]. Therefore, without these protocols, IBMs and ABMs were reported with varying levels of detail and often in insufficient detail to understand all of the modeling choices. The ODD framework elicits critical descriptives of the model design and development and the ODD + D protocol added detailed guidance on how to report the decision-making elements in the development framework, which was particularly important for ABMs. We believe that the $\mathrm{ODD}+\mathrm{D}$ framework offers a valuable approach to extracting and understanding model development and model comparison across studies and encouraging wider use of this framework by other ABM modelers, particularly in the social sciences. 
The lead author for this review extracted the data points from all 28 articles using comma-separated value (CSV) files according to key questions in the ODD $+\mathrm{D}$ protocol. The extraction table was quality-checked by all three co-authors in both workshop-style review sessions and independent reviews.

\section{Data synthesis}

Since the majority of the data points extracted for this review were descriptive characteristics of the ABMs, the data extraction table is largely qualitative and too large to present in article format. In this same respect, the challenge to qualitative data synthesis in systematic reviews also applies to this methodological

Table 3 Results synthesized by sub-topic and presented in individual tables

\begin{tabular}{|c|c|}
\hline All tables & Lead author (Year) \\
\hline Table 4: Included articles & $\begin{array}{l}\text { Author(s) } \\
\text { Year } \\
\text { Title }\end{array}$ \\
\hline Table 5: Study characteristics & $\begin{array}{l}\text { Authors' institutional affiliation(s) (country) } \\
\text { Authors' department(s) } \\
\text { Journal } \\
\text { Study purpose as summarised by reviewers (aim sub-category } \\
\text { classification) }\end{array}$ \\
\hline Table 6: Model inputs & $\begin{array}{l}\text { Decision model } \\
\text { General concepts or theoretical basis for model design } \\
\text { Data type } \\
\text { Data source }\end{array}$ \\
\hline Table 7: Model development & $\begin{array}{l}\text { Time-steps and time-horizon } \\
\text { Spatial characteristics } \\
\text { Agent types: Agent attributes [micro characteristics] } \\
\text { Exogenous or environmental characteristics [macro charac- } \\
\text { teristics] } \\
\text { Social networks [meso characteristics] }\end{array}$ \\
\hline Table 8: Model process & $\begin{array}{l}\text { Initialisation } \\
\text { Model steps and decision rules } \\
\text { Agent types: Agent actions } \\
\text { Simulation scenarios } \\
\text { Deterministic or stochastic (if stochastic: which model com- } \\
\text { ponent) }\end{array}$ \\
\hline Table 9: Model analysis and validation & $\begin{array}{l}\text { Output variables } \\
\text { Uncertainty and sensitivity analysis as described by the } \\
\text { author(s) } \\
\text { Model validation as described by the author(s) }\end{array}$ \\
\hline
\end{tabular}




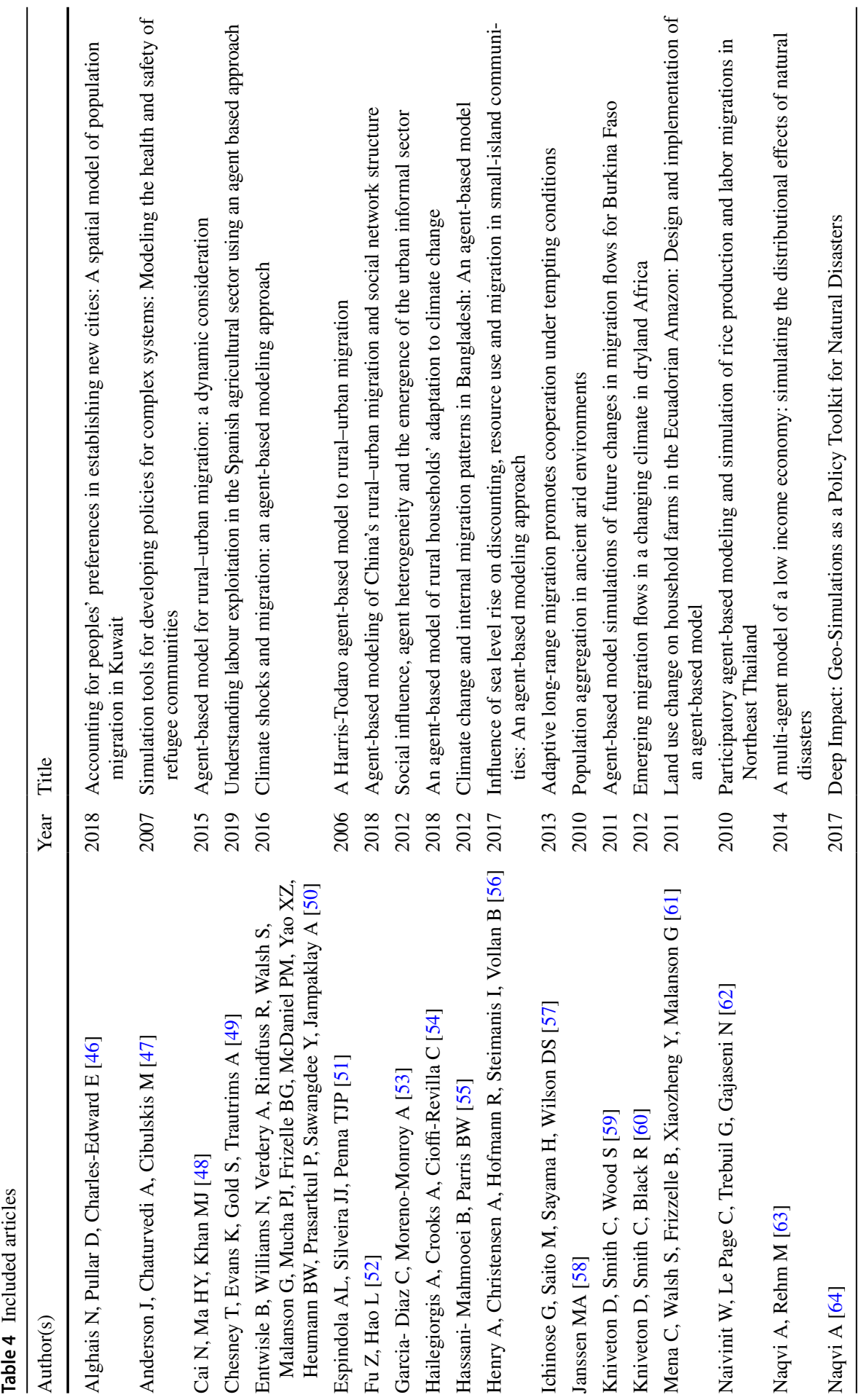




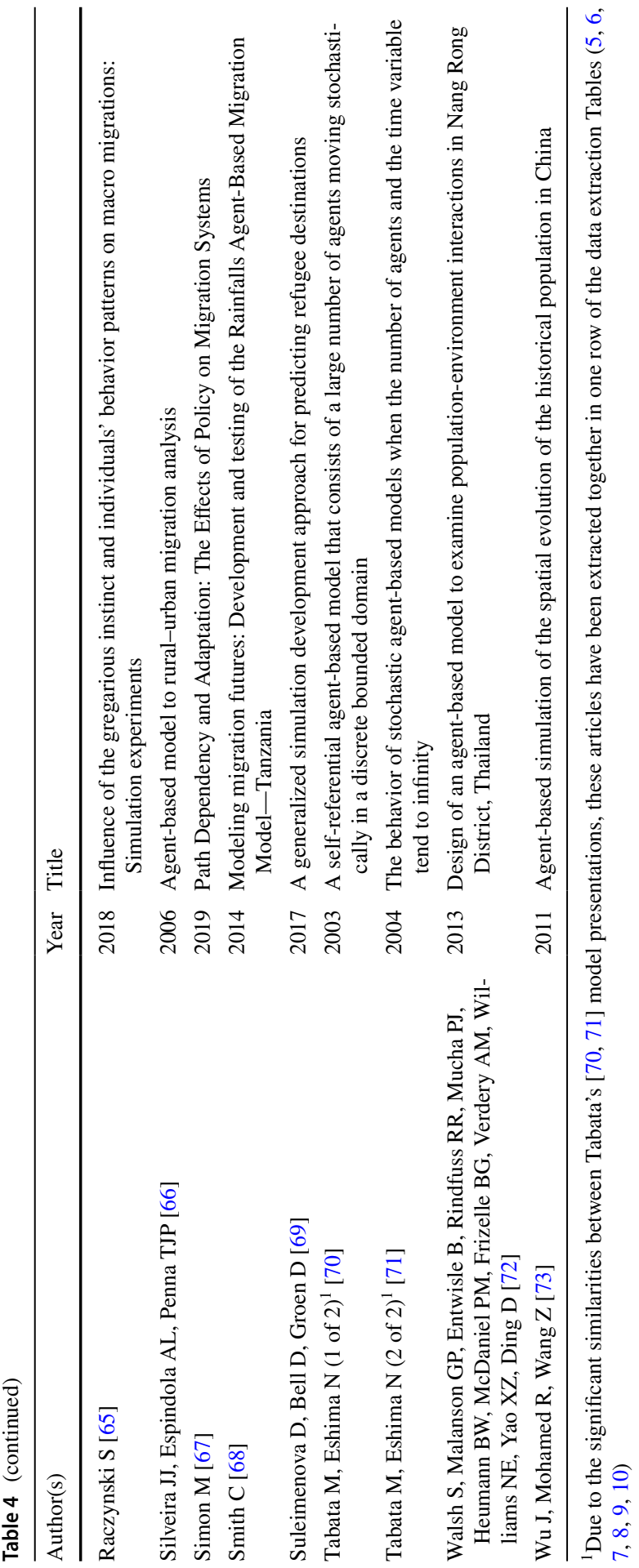


review [44]. When possible, we have categorized certain data extraction points to more easily summarize or draw conclusions on the range of methodological choices made by the authors. Therefore, we have grouped a majority of the data extraction into seven results Tables (4, 5, 6, 7 8, 9 and 10) and presented our data synthesis for each table (See Table 3 for summary of results tables). The synthesis includes categorizing and summarizing the key data extraction points to make comparisons and highlight gaps across all 28 ABMs. The full data extraction table can be found in the Supplementary Materials (SM-3). We did not assess the quality and suitability of ABM methods used in the included studies because it is outside the scope of the review. This is in part because, to our knowledge, there is not currently a standardised instrument to assess the quality of ABM models like the tools that exist for assessing other research methods, such as The Critical Appraisal Skills Program (CASP) tool [45].

\section{Results}

In total, we identified 137 articles on relevant migration or modern slavery research topics that applied either network analysis or dynamic simulation methods. This included research using social network analysis, Bayesian network analysis, system dynamics modeling and various microsimulation methods. We then further excluded any article that did not use ABM methods. We identified 28 articles, included in this review, which explicitly detailed ABM (or 'Agent Based Simulation') in the methods (Table 4).

This results section presents: the data extraction and synthesis of the study characteristics (Table 5); model inputs (Table 6); model development (Table 7); and model process (Table 8); model analysis (Table 9); and the summary of the model aim in relation to specific model development characteristics from Tables 5, 6, 7, 8, 9, 10. But first, we will present two important observations made during the screening and extraction process that are relevant to inferences about methodological procedures in ABM, one of the key objectives of this review:

(1) Agent-based models are sometimes called by different names;

(2) Few studies reported using ODD $+\mathrm{D}$ or an alternative development framework.

1. Agent-based models are sometimes called by different names

Similar to findings from Klabunde's and Willeken's review, it was challenging to ascertain whether some dynamic simulation models (e.g. spatial or dynamic microsimulations, individual-based models) that were not explicitly called $\mathrm{ABM}$ (or ABS) did in fact include sufficient agent-interaction or decision-based behavioral modeling to be included as ABMs [2]. This challenging grey area of inclusion is not altogether surprising, since expert commentaries on the origins of ABM explain that cellular automata, microsimulations, and agent-based approaches have been developed and improved on in parallel, across disciplines, with different aims, and different names, but seemingly similar function and capabilities $[74,75]$. For example, microsimulations are often considered to be 
the method of choice when exploring policy impacts and ABM are used more commonly for theory testing. Distinctions aside, these models all have bottomup approaches that model individual behaviors, often heterogeneous [75]. While at times the distinction appears arbitrary, for this systematic review, we decided to apply strict criteria that the paper had to identify the methods as ABM to be included in the extracted yield of articles. In part, this is to ensure the reproducibility of the review, but also to allow for as uniform and complete data extraction of methodological processes as possible, which we determined would be more feasible if the authors were writing their methods from an ABM framework. We have included a table in the Supplementary Materials (SM-2) with a list of the 8 articles we determined were implicitly describing ABM but were excluded for the reason just described.

2. Few studies reported using ODD $+\mathrm{D}$ or an alternative development framework

Although the majority $(n=24)$ of the included articles were published after the introduction of the well-known 2006 ODD framework for simulation or the 2013 ODD + D framework for ABMs (See Table 4 for publication years), very few of the articles described using any framework in developing or reporting the ABM. Only one study used the ODD + D framework designed specifically with ABMs in mind [54], 3 studies used the ODD framework [46, 55, 62], and one used a framework developed by the authors themselves which they called a generalized Simulation Development Approach (SDA) [69]. This review, as noted, used the ODD + D framework to inform the data extraction tool, in part, because we hoped a majority of recent ABM research would have used this framework. It appears that the uptake of this framework tool is still slow and this continues to create challenges for understanding model inputs, development and analysis as well as comparison across studies. We note this before addressing the bulk of the synthesis of the study methods to inform the reader that the extraction process had to adapt to a wide range of styles in disseminating the model development process.

Below are the main results of this review according to the six sections described above in Table 3.

1. Study characteristics

Between 1999 and 2019, the publishing years of inclusion in this review, there have been a steadily increasing number of studies using agent-based modeling to investigate the migration and modern slavery topics covered by this review (See Fig. 4).

The authorship characteristics, both by institutional affiliation and by disciplinary associations, represented a diverse range of home institutions, country of institutions and disciplinary departments or research centers (See Table 5). Nine of the included studies were authored by multi-country authorship teams and 


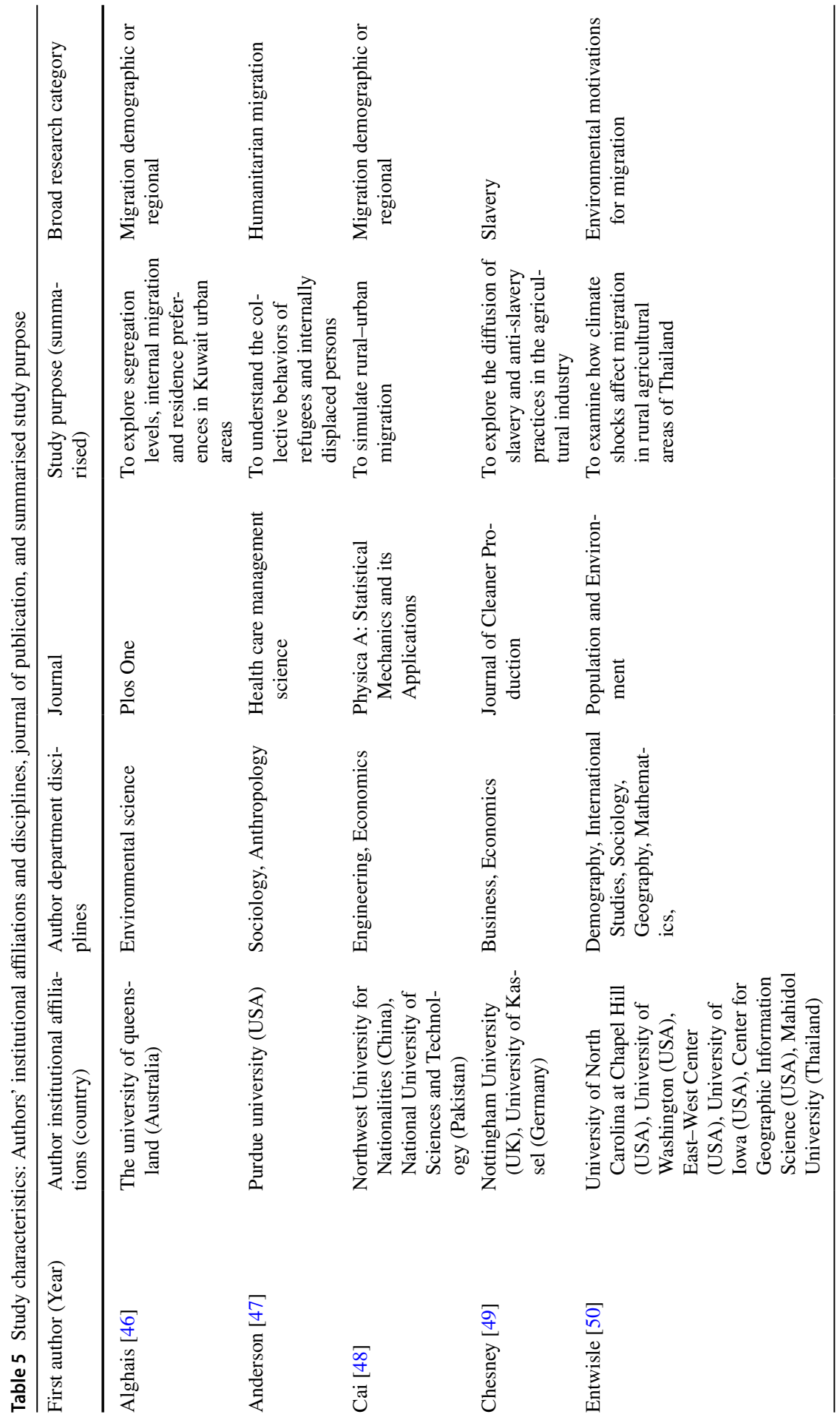




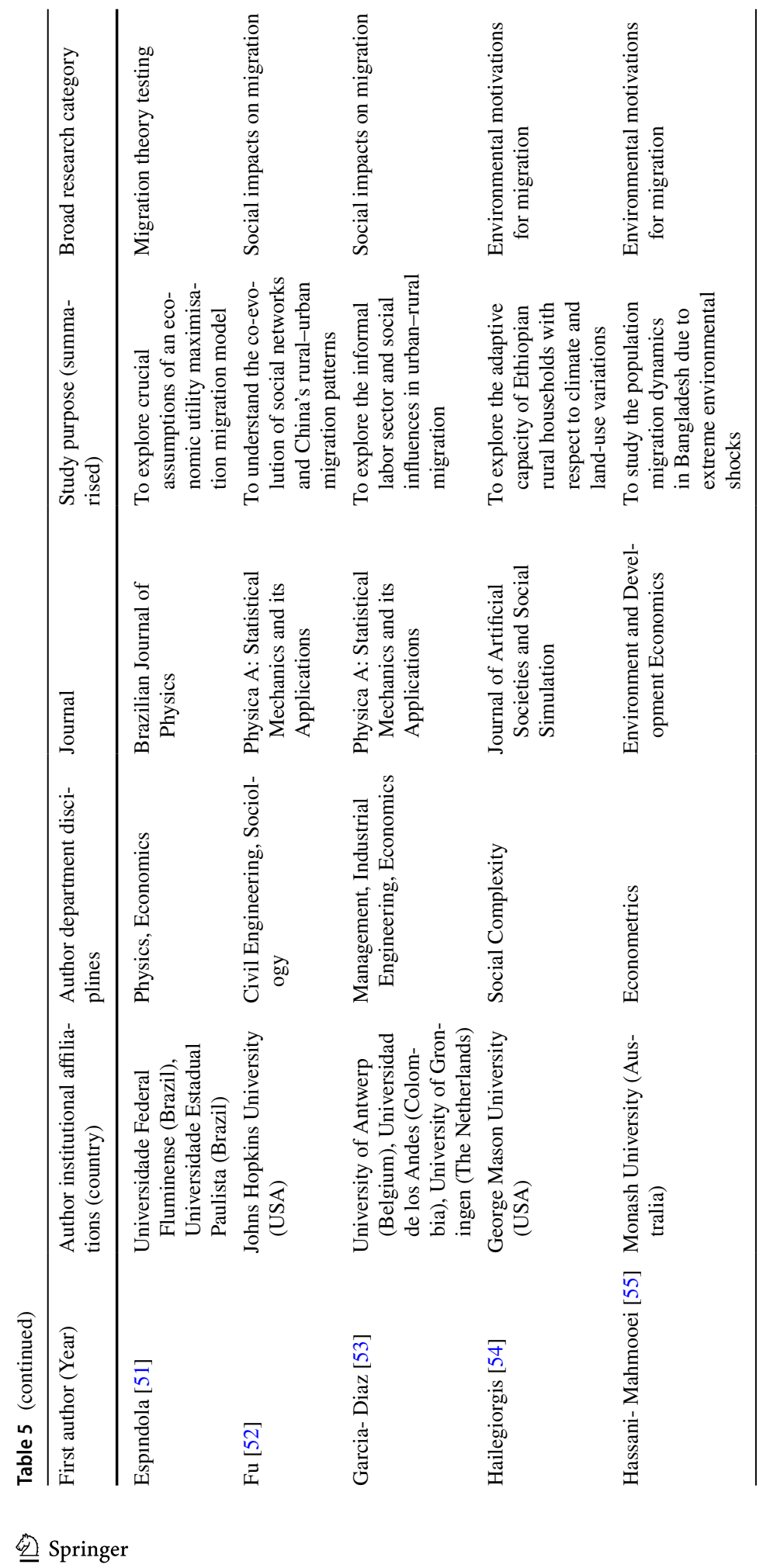




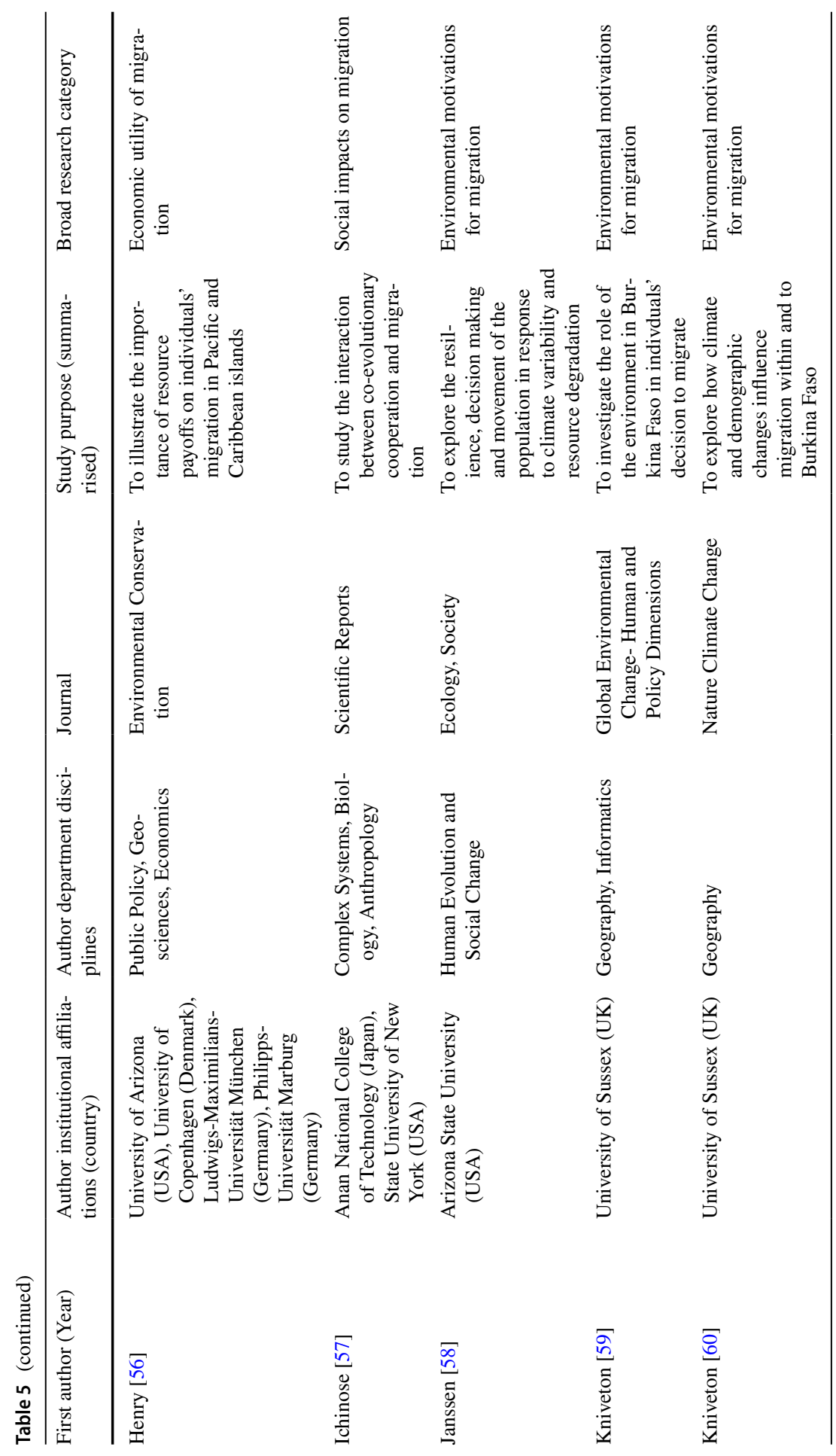




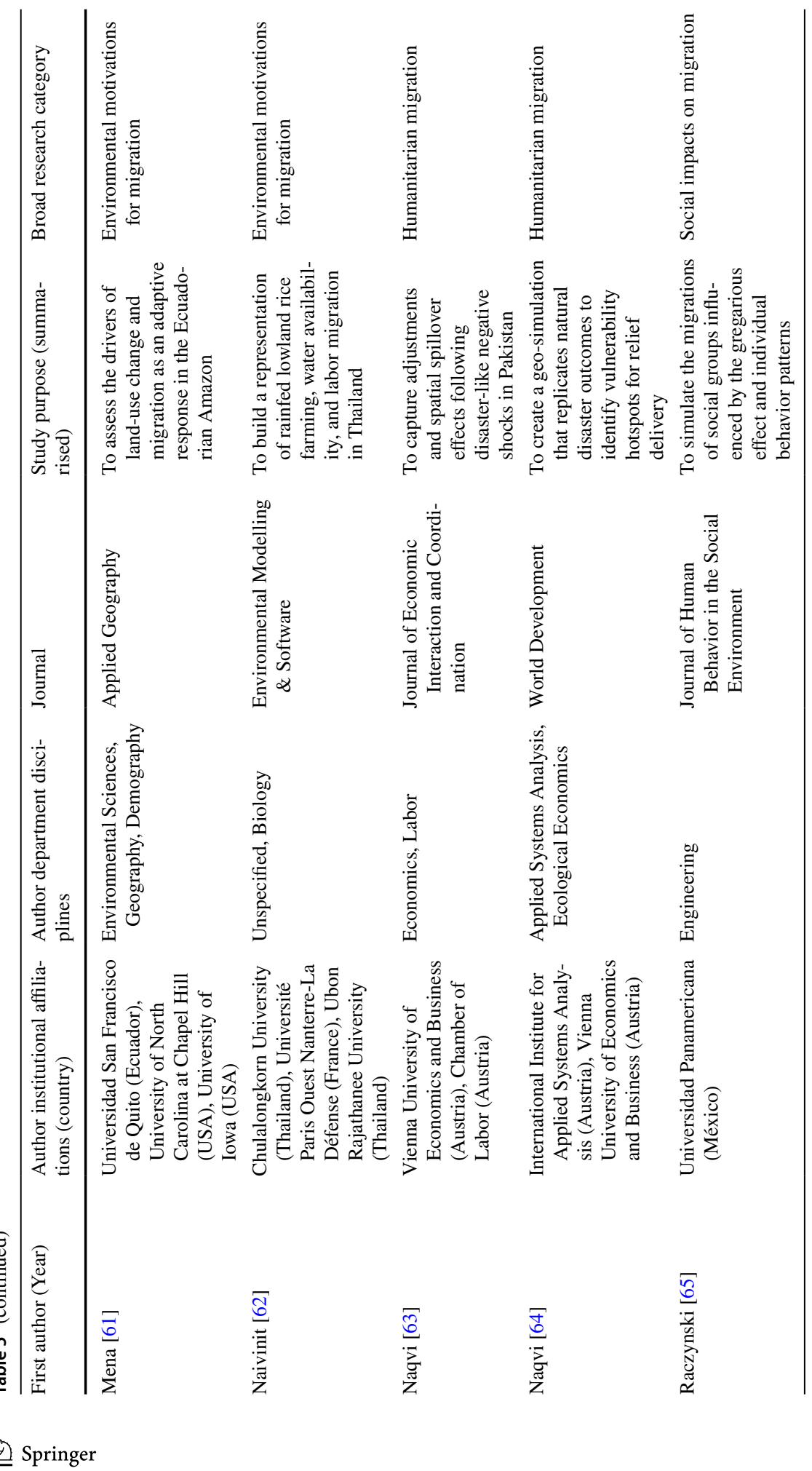




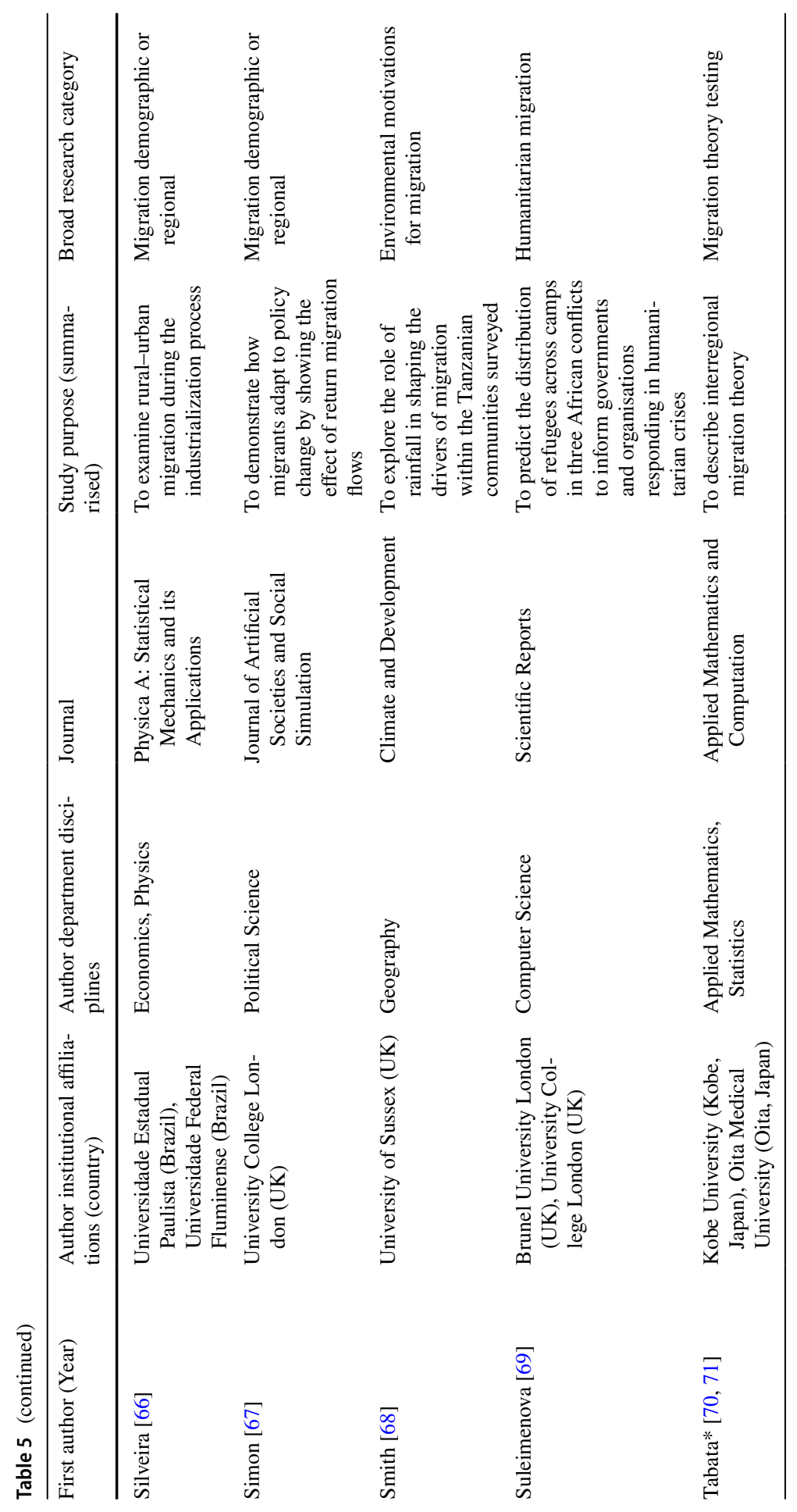




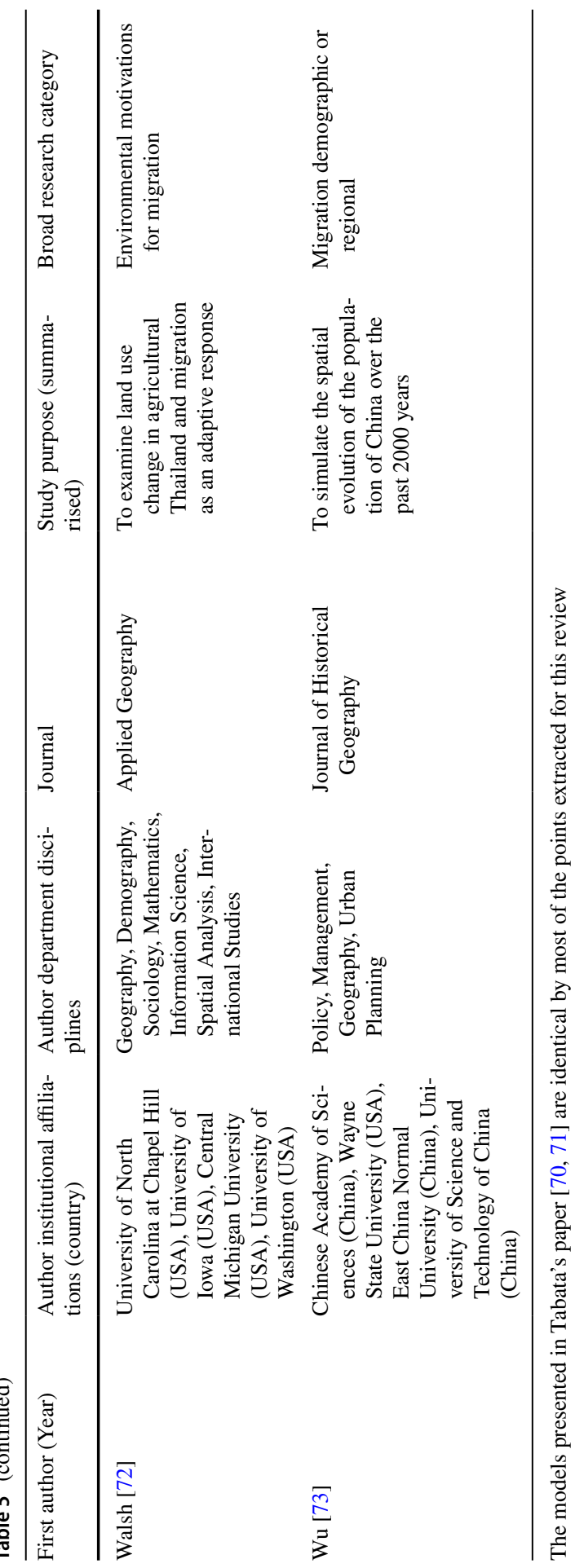


thirteen of the included articles indicated cross-disciplinary authorship teams. It is also noteworthy that ten of those cross-disciplinary authorship teams consist of both "hard sciences" (mathematics, engineering, physics, etc.) and "soft sciences" (sociology, anthropology, economics, demography, etc.). For example, the Walsh et al. article was authored by scholars from backgrounds in mathematics, information science, spatial analysis, geography, demography, sociology, and international studies, who could each bring his or her disciplinary expertise to the task of mathematically modeling environmentally influenced migration patterns [72].

For each study, the aim defines how to calibrate the model (choosing the data sources and applying various data sources to different elements of the model) and how to develop the model (the time, space, agents and environment, as well as the model decision rules). The included studies present a range of exploratory aims, which we have grouped into seven broad sub-categories: migration demographic or regional trends; environmental motivations for migration; migration theory testing; humanitarian migration trends; the social influence on migration patterns; the individual economic utility of migration; and slavery. The two most frequent research categories were: exploring the environmental motivations for migration, such as climate change, environmental shocks or land-use changes $(n=10)$; and explorations of migration corridor trends by geography or demographics $(n=5)$. The other study aims fell into the following categories: humanitarian migration trends $(n=4)$; social influences on migration patterns $(n=4)$; migration theory testing $(n=3)$; individual decision-making based on the economic utility of migration $(n=1)$; and the diffusion of slavery $(n=1)$. None of the studies explicitly aimed to understand various migration pathways and individual processes of migration, since most were focused on macro-drivers of migration trends, such as expected wage differentials, weather conditions or humanitarian situations.

2. Model inputs

Most studies used multiple types of data (theory, primary, secondary, historical, cited research, reports, etc.) to calibrate the model parameters, initial settings, environmental characteristics, scenarios or decision rules (See Table 6). Recently, the computational social science community has begun to devote more attention to empirical calibration and validation of ABMs [76], where previously the vast majority of ABMs have been calibrated solely as thought experiments using theory. In this review, 5 articles reported using primary empirical data for ABM calibration and 11 studies used secondary data sources (excluding GIS data, historical climate data, or cited research findings as 'secondary data'), an approach that some have called an 'indirect strategy' for empirical calibration that is a limited but promising option when collecting primary empirical data is not feasible. The notable difference between the studies that used primary versus secondary empirical data is that all 5 studies that collected primary data used the analysis of this data to inform individual behavioural rules in the model (e.g. how migrants decide to migrate) but only 5 (of 11) of the studies that only used secondary data sources did so to inform individual behavioural rules. Both primary and secondary empirical data sources were used to inform agent attributes $(n=2)$, population size and attributes $(n=4)$, population trends (e.g. migration flows, household crop yields, household spending) $(n=5)$, social networks $(n=1)$, 
regional land use $(n=3)$ and key events (e.g. natural disasters, conflicts) $(n=2)$. The use of empirical and secondary data sources in this review reflects the wider trends in empirically calibrated ABMs, which is usually more common to find secondary data that can inform the model structure components (types of agents, number of agents, environmental characteristics) but less common to find secondary data sources that offer the rich data on behaviors and interactions that are often so central to the theoretical research questions [76].

Most commonly $(n=21)$, the ABM decision rules or assumptions were informed by theory, primarily from economics, psychology or theoretical developments in the field of migration studies. In fact, 9 of the ABMs exclusively used theories to calibrate the models, such as theories on the push and pull factors of migration or the influence of social networks on migration, to calibrate the model, as can be reviewed in Table 6. This is not surprising given the scarcity of migration data collected from a complex systems approach that would be easily translatable to ABMs. For example, ABMs modeling labor migration as a complex system would need data on migrants' social networks' role in the migration process, the interactions between migrants and the wider systems environment, or the decision-making process of migrants, labor intermediaries and employers. There is a high burden to collecting this kind of data in any setting, and there are additional challenges in research with a largely hidden and mobile population, such as low-wage international migrant workers or human trafficking victims [77, 78]. Boero and Squazzoni point out that despite the challenges to missing, incomplete or scarce data in many fields of research, the valuable theoretical constructs produced using ABM methods need to be embedded in empirical findings (at both calibration and validation stages of model development) so that the theoretical mechanisms are empirically grounded in the real-world phenomena [76].

Studies that employed secondary datasets $(n=15)$ sourced the datasets from various federal and municipal governmental departments, as well as national and international non-government organisations, such as the World Bank or United Nations $[47,59,60,63,64,69]$. For spatial calibration of the model, eight ABMs used some type of GIS data. Only one study used participatory methods in calibrating the model with key stakeholders by hosting ABM workshops at various stages in the model development [62]. Additional data sources included in-depth case studies, secondary research findings in the literature, non-peer-reviewed reports and historical trends in weather or migration (See Table 6). The models pulled from a wide range of theoretical knowledge and empirical analysis to inform model development and decision-model choices. For example, the range of theory and background analysis included psychology-based theories of wellbeing and decision-making, information diffusion, cooperation theory, systems theory, social network theory, topics in microeconomics, game theory and a range of topics pertaining to migration push-pull factors (See Table 6).

The model's data inputs often determine the decision model used in an ABM. An ABM can employ more than one decision model, such as a combination of simple heuristics (breaking down agent actions into simpler if/then rules) and decision theory (generalized knowledge on human's reasoning and process in making decisions) or microeconomics utility maximisation (the aim is maximis- 


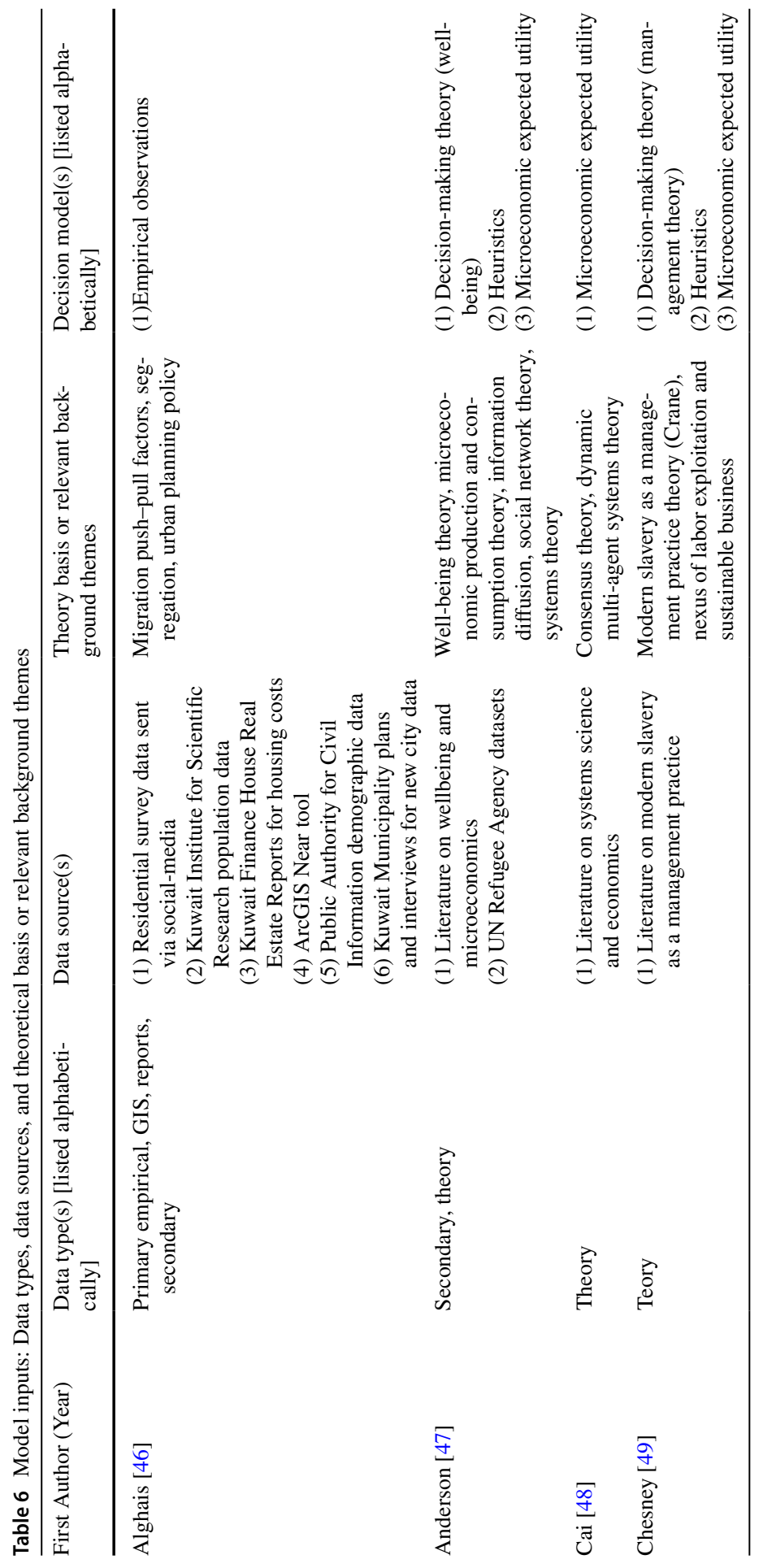




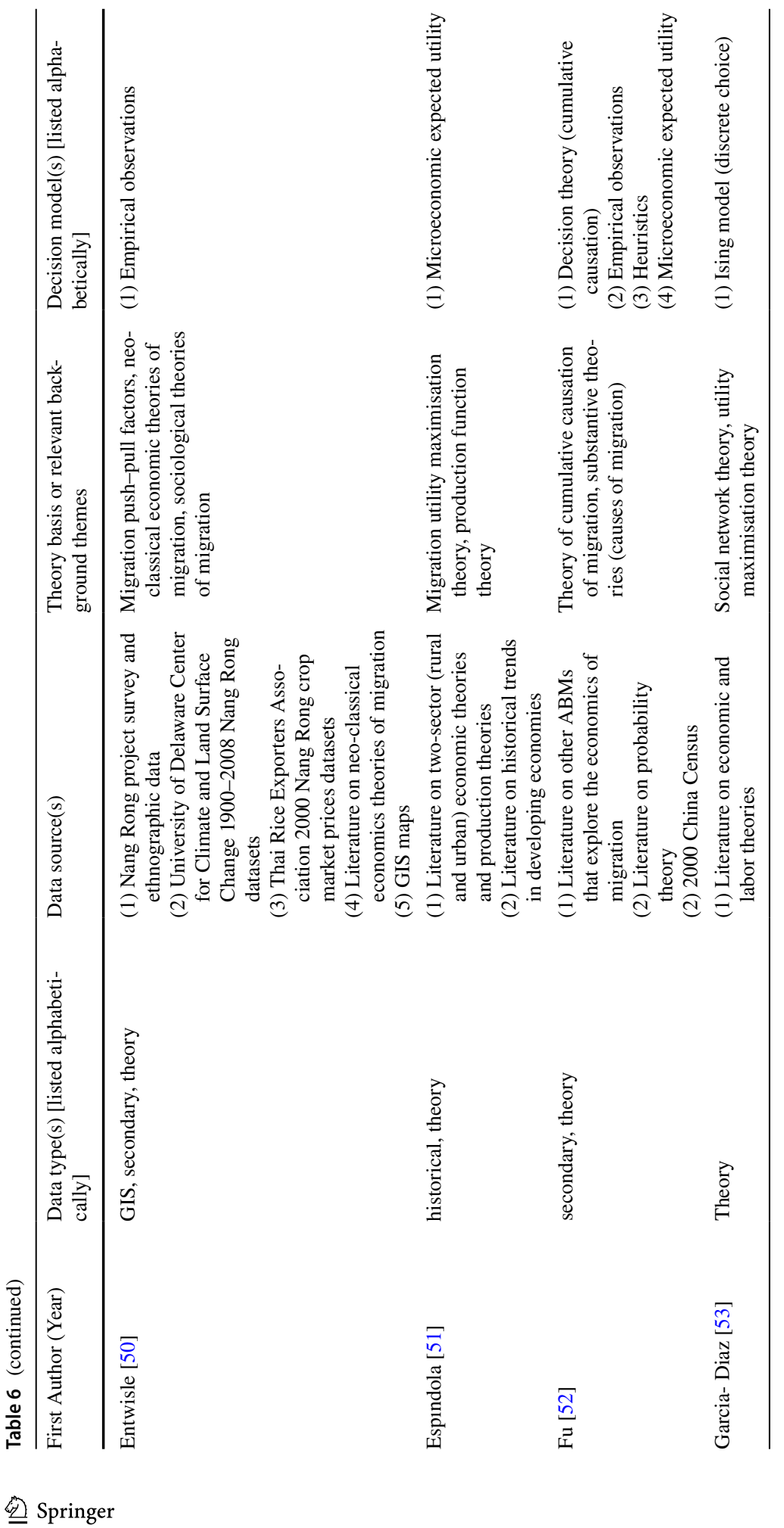




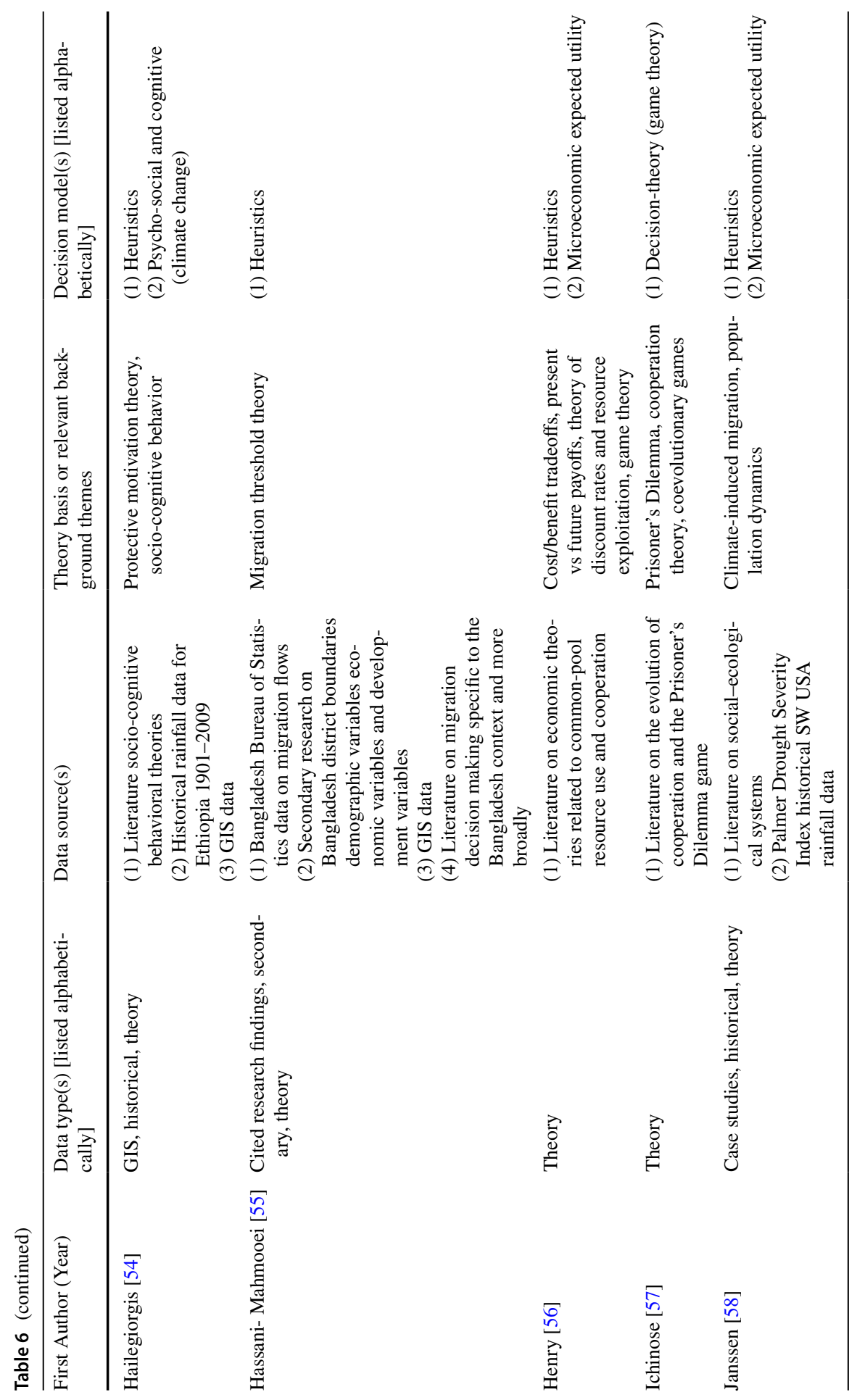




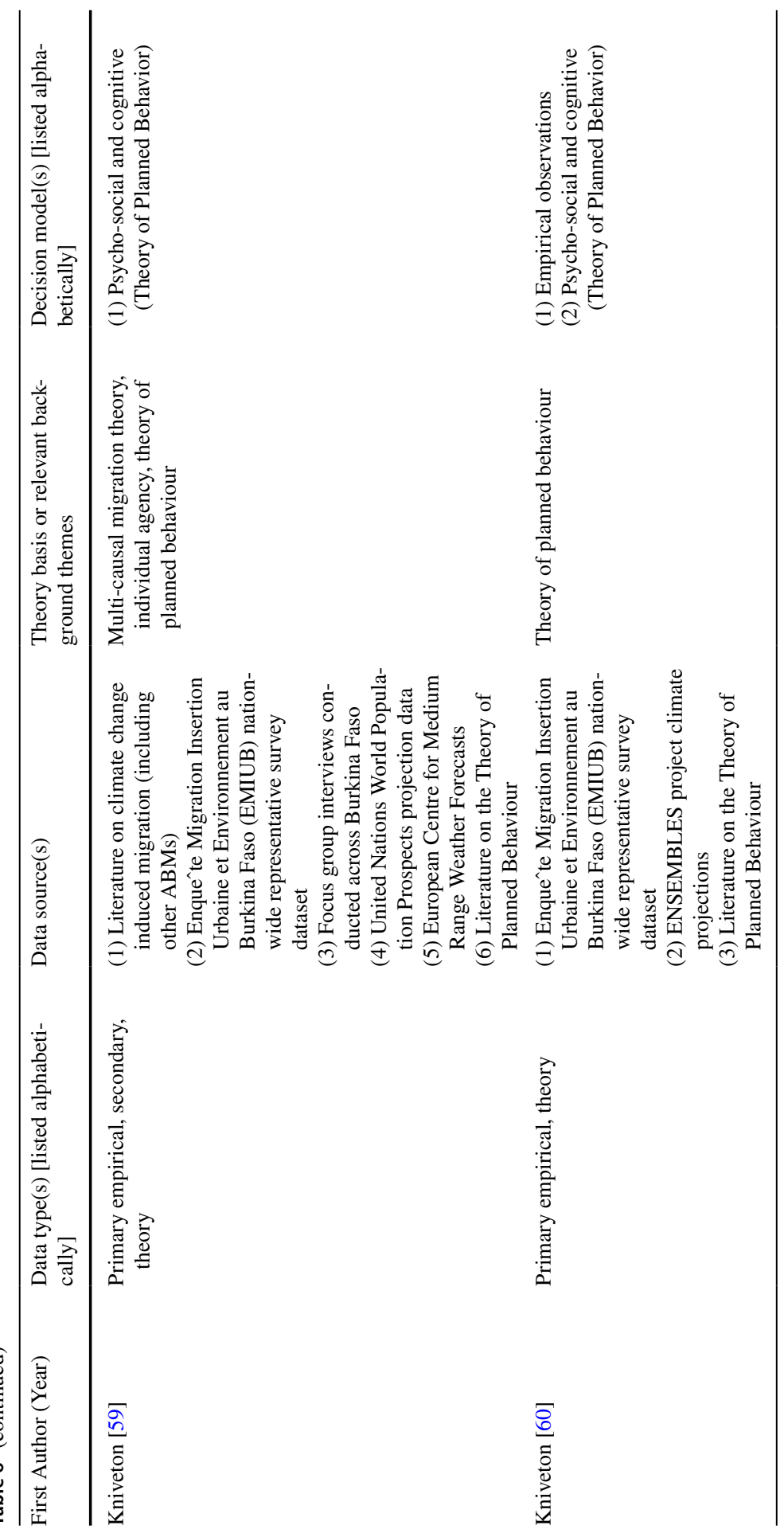




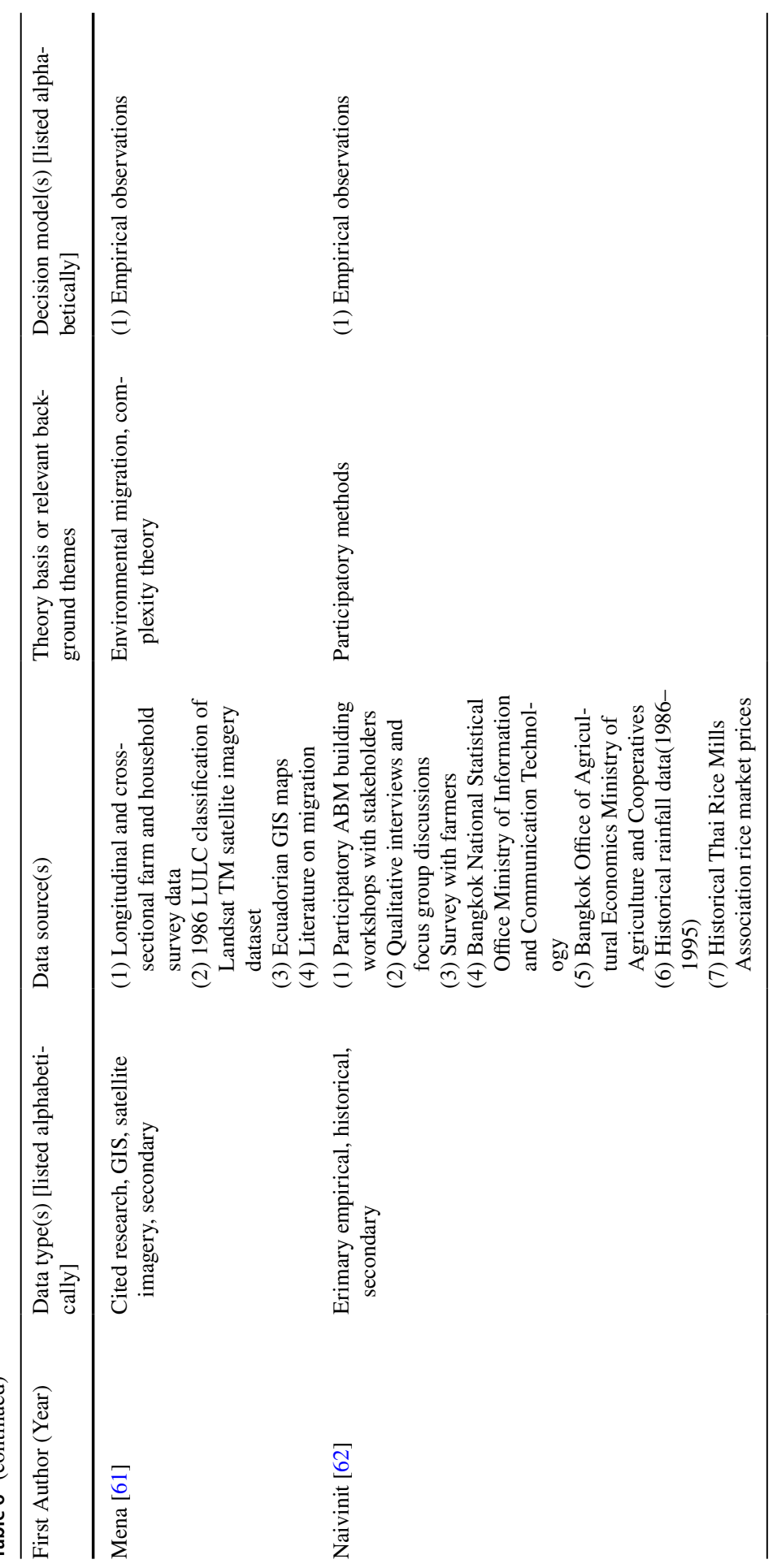




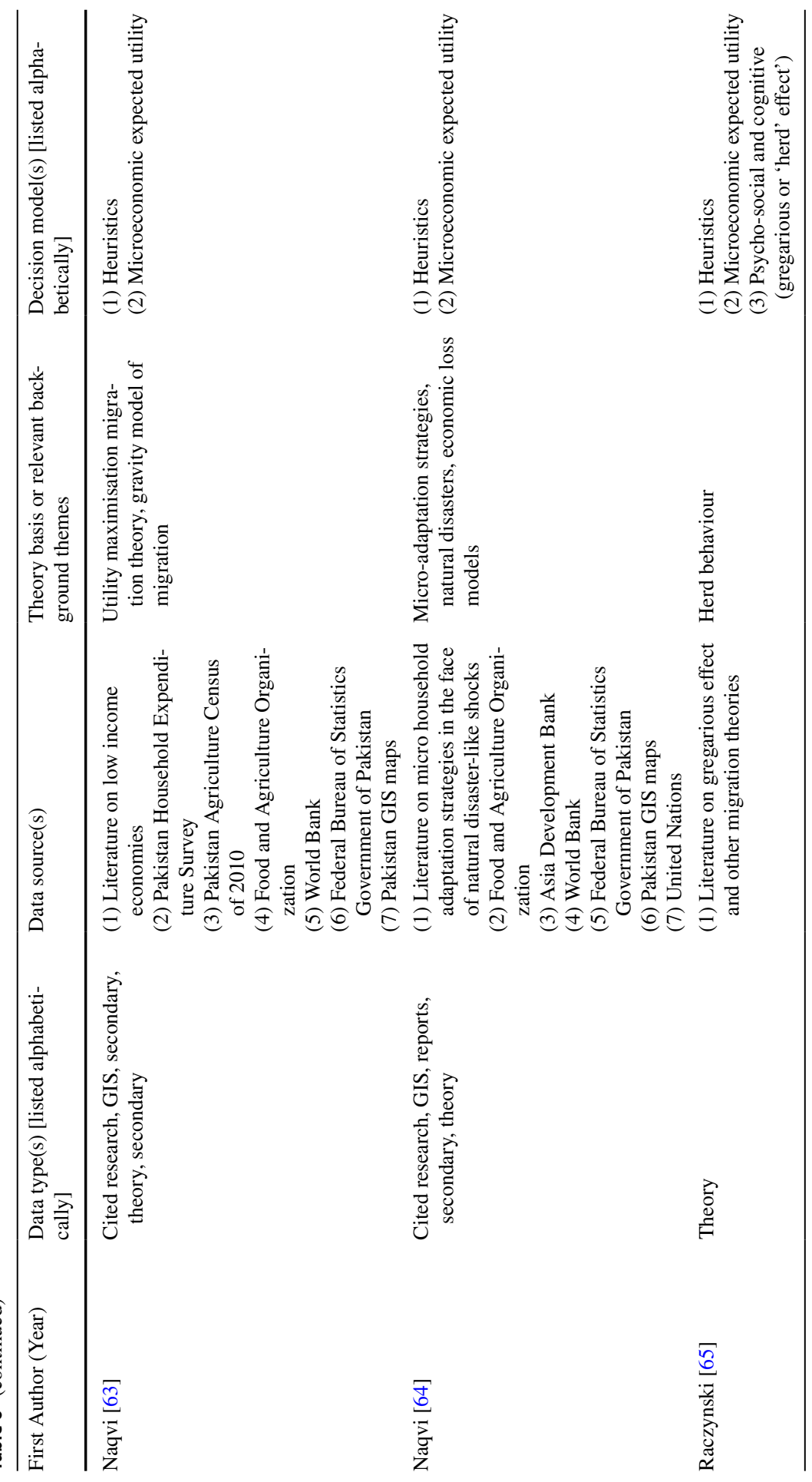




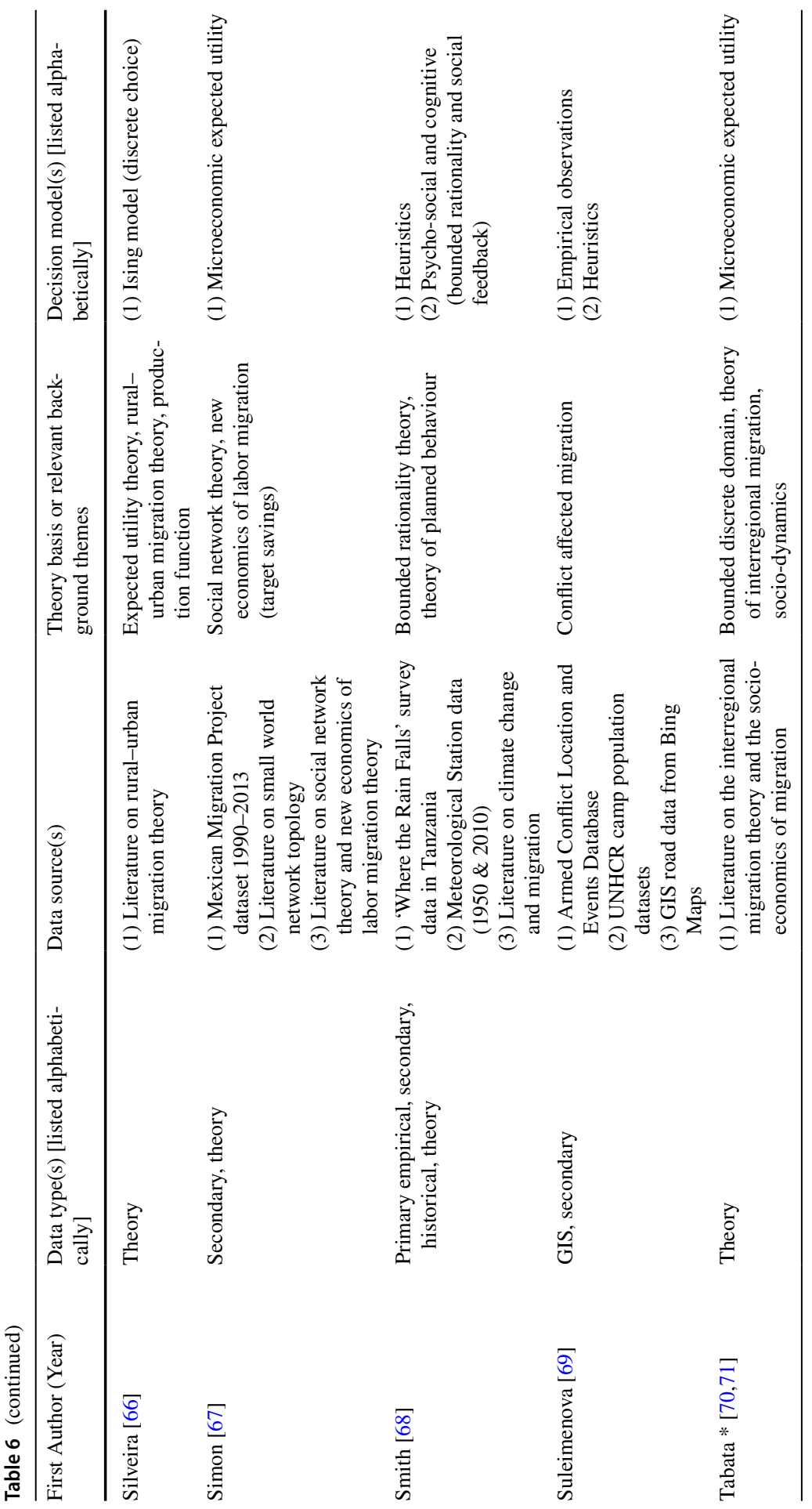




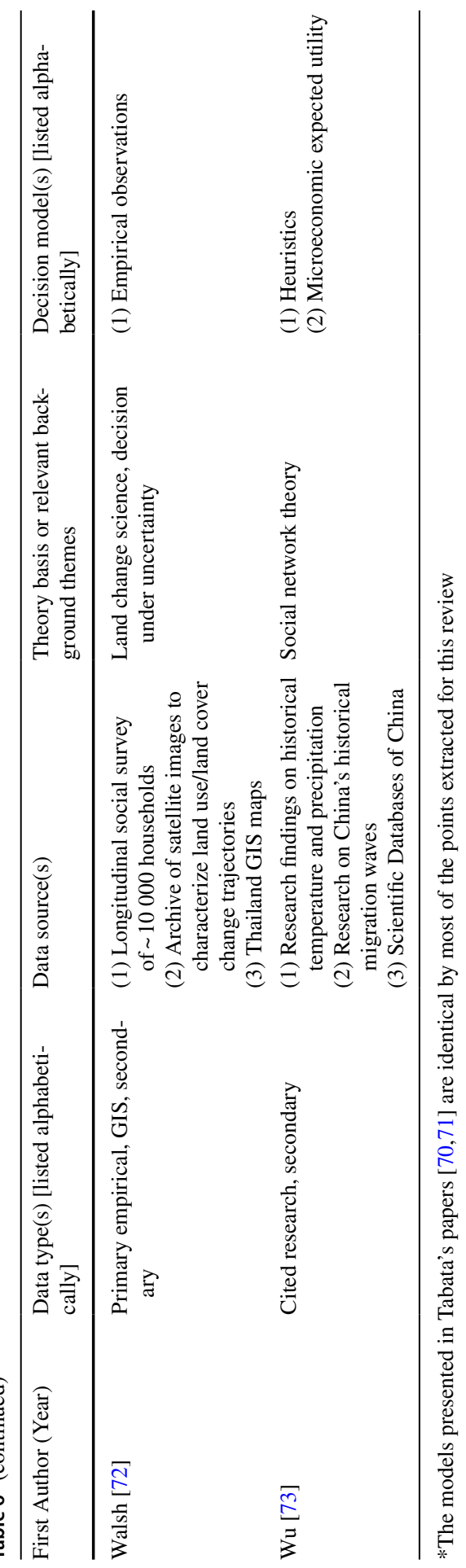


ing profits or payoffs, such as wage differential motivated migration). Or in some cases, there might be some use of empirical data, but the data are often insufficient to inform all the decision-making processes so theoretical knowledge is used in combination with empirical observations. For example, Alghais, Pullar and Charles-Edward used primary empirical survey data from urban residents in Kuwait on their residential preferences to determine empirically informed decision rules for why, when and where individual agents would migrate to new urban centers [46]. Decision-making was also the primary focus of Klabunde and Willekens's review and they give an extensive description of the different decision models currently being used in migration ABMs similar to the decision models described above. This review borrows some of Klabunde and Willeken's decisionmodel categorisations in Table 6 [2]. All eight studies that collected primary data also incorporated these data into the ABM's decision model. Microeconomic utility maximisation has been a long-standing theory applied to the study of migration motivations and decision-making and was used to model decisionmaking in 12 of the included studies (See Table 6). As Klabunde and Willekens's review points out, there are also key theories pertaining to both individual and group decision-making and pyscho-social and cognitive behavioral science that are increasingly being applied to the study of migration, such as the Theory of Planned Behaviour [2]. In our review, four studies described the using a range of specific decision-making theories in their decision-model choices, which covered broad topics of well-being [47], management [49], cumulative causation including the role of social influences [52], and game theory [57]. Additionally, both of the Kniventon et al. studies applied the Theory of Planned Behavior, which is considered a psycho-social and cognitive theory and described in further detail in Klabunde and Willekens's review [2]. Three other studies described the use of a psycho-social and cognitive theory in the decision model. The psyco-social and cognitive theories incorporated the influence of climate change [54], the herd effect [65], and a combination of bounded rationality and social feedback [68]. Commonly used decision models were simple heuristic models $(n=13)$ that were not always informed by any primary empirical findings nor based on clearly defined theoretical knowledge.

3. Model development characteristics

ABMs incorporate the attributes and interactions of agents, time, space, environment, and in some cases social networks and exogenous factors. The majority $(n=21)$ of the included studies defined the time-steps in terms of real time ranging from $1 \mathrm{~h}$ to 5 years and the time horizons ranging from 4 days to 10,000 years (See Table 7). The 10,000-year time horizon was a far outlier since it was a historical anthropological migration study. Most of the studies had time horizons that were 50 years or shorter. Seven of the studies used time scales that reflected real historical or date ranges, primarily in years, such as Kniventon, Smith and Black's model on climate induced migration in Tanzania from 1970 to 1994 [60]. The remaining models that included clear explanations of time-steps $(n=4)$ represented these discrete increments as 'time-to-event' meaning that they represented distinct processes, such as farming seasons or migration cycles [51, 53, 56, 57]. 


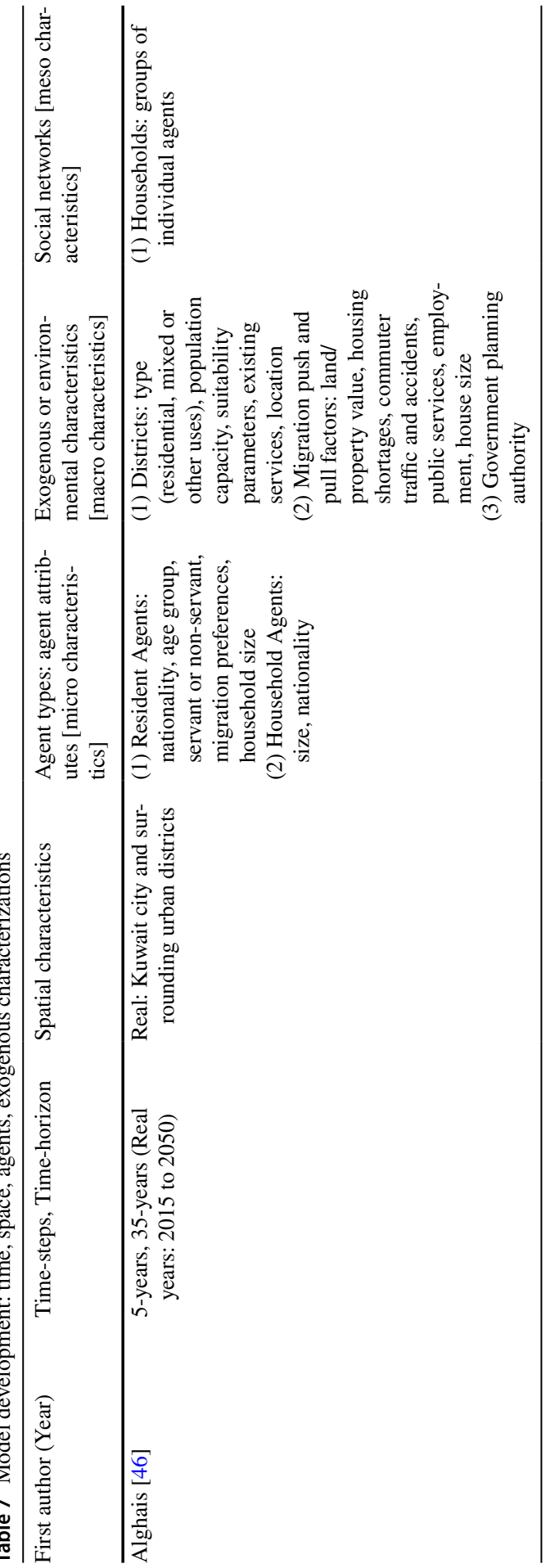




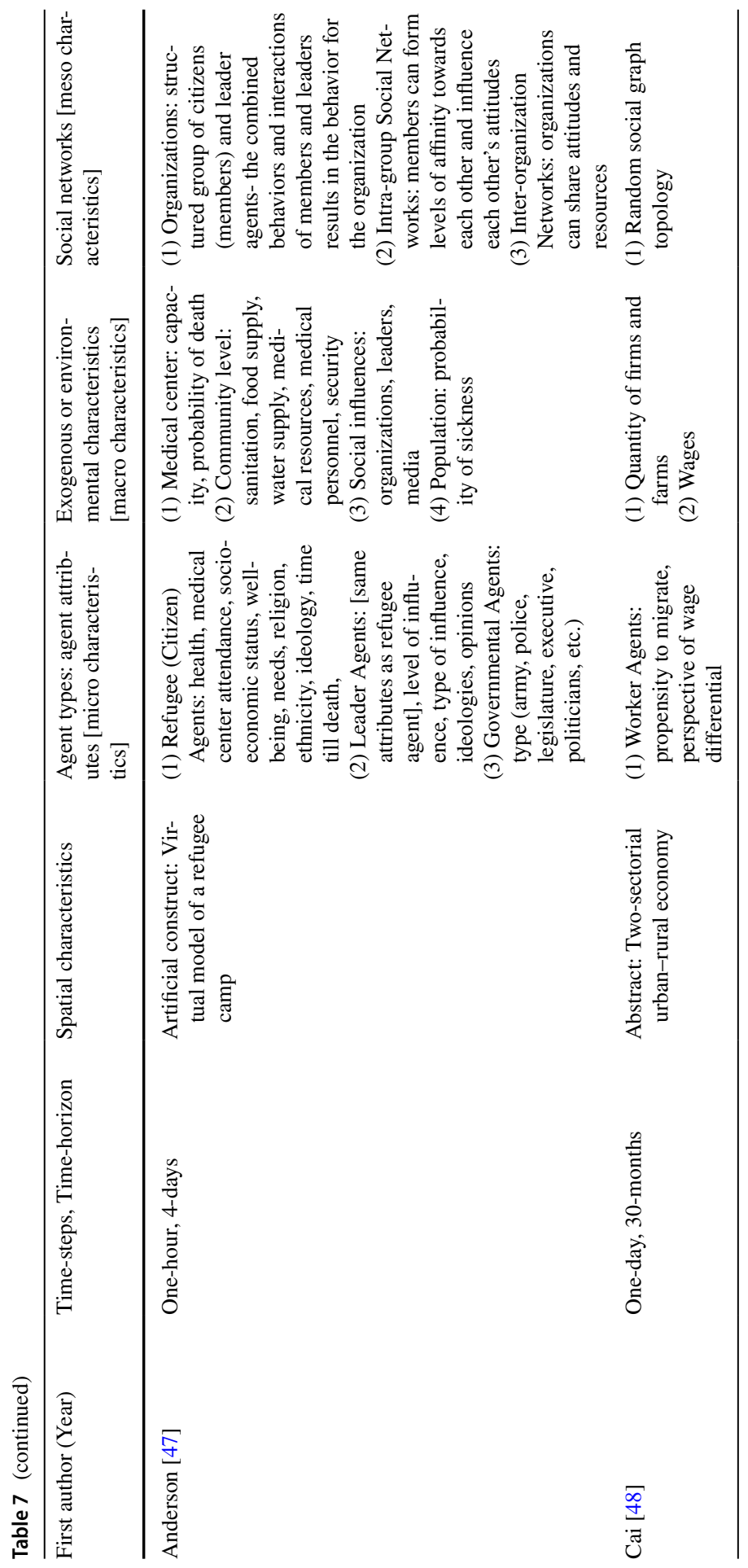




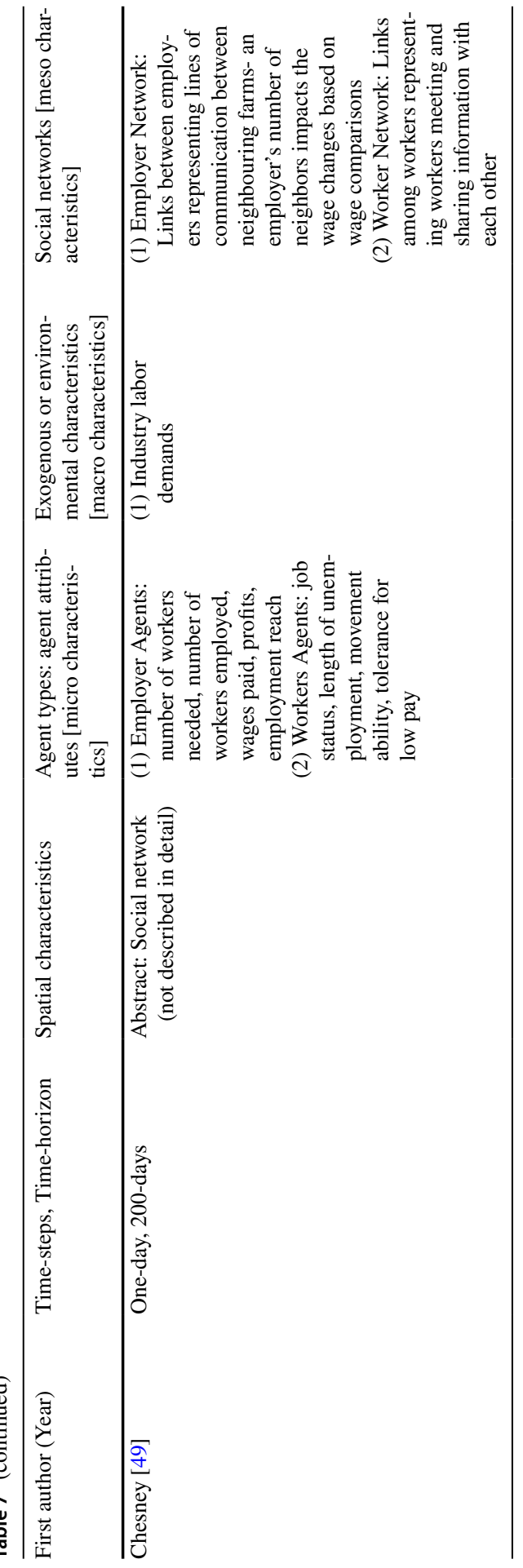




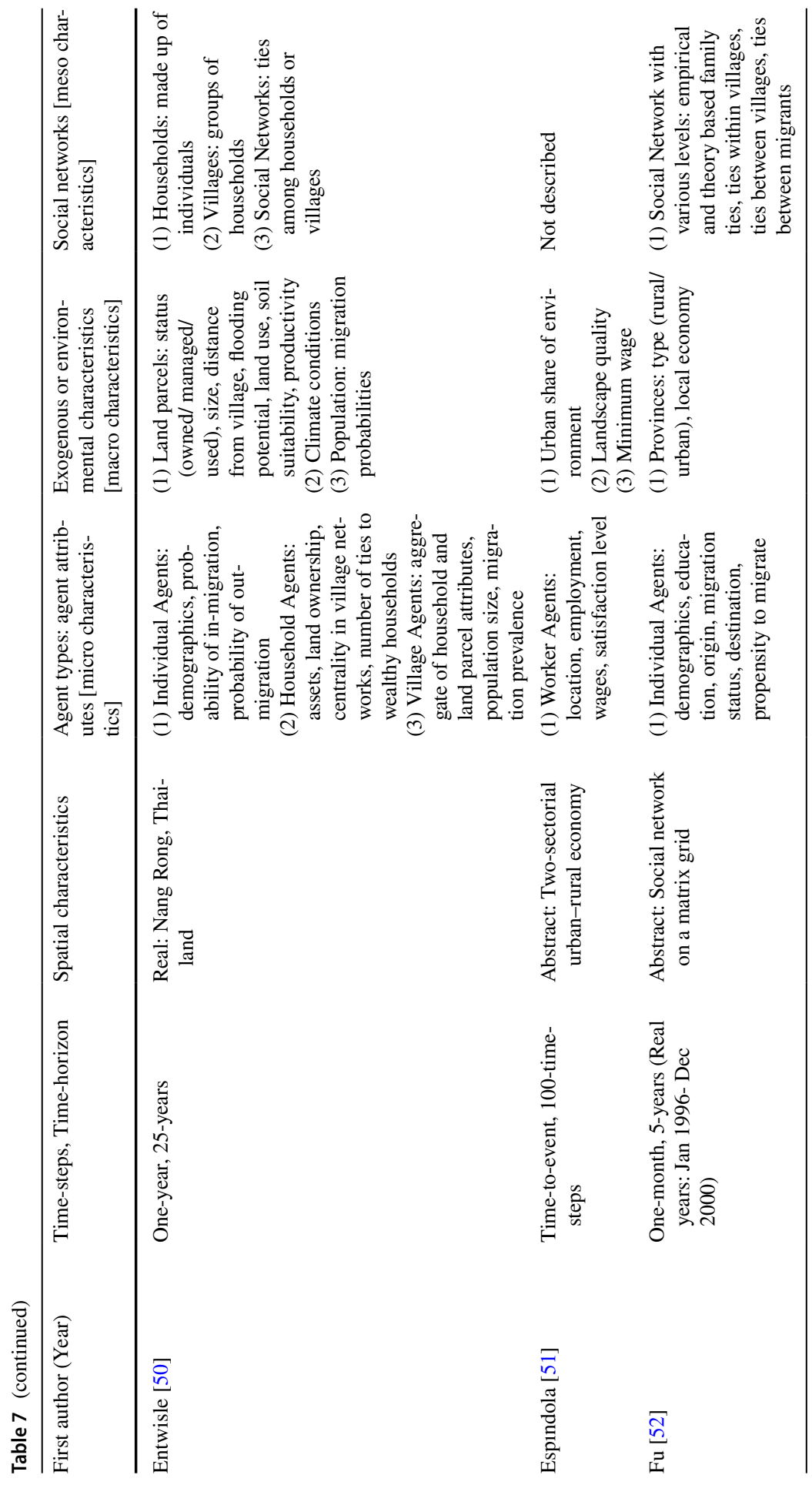




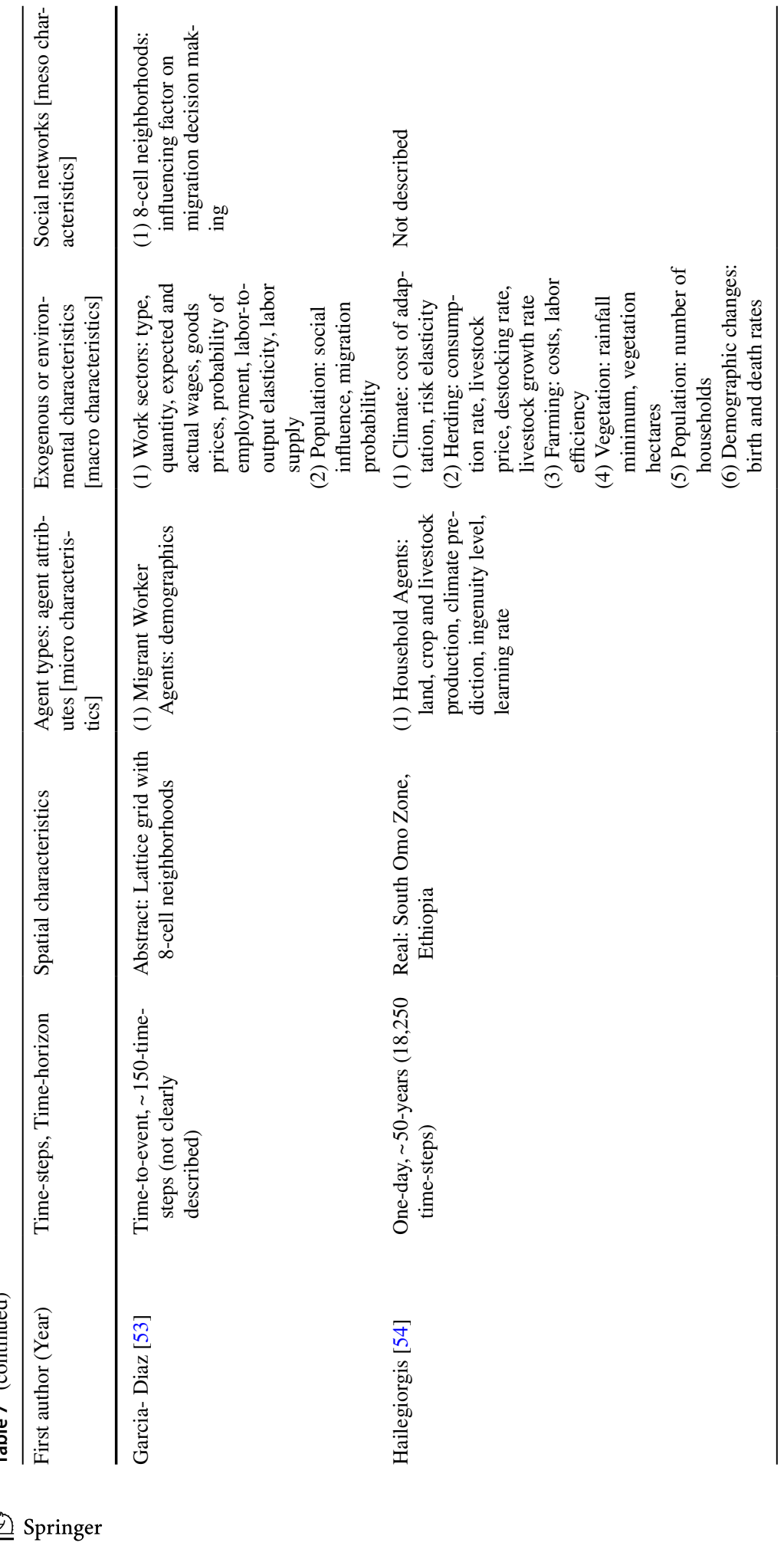




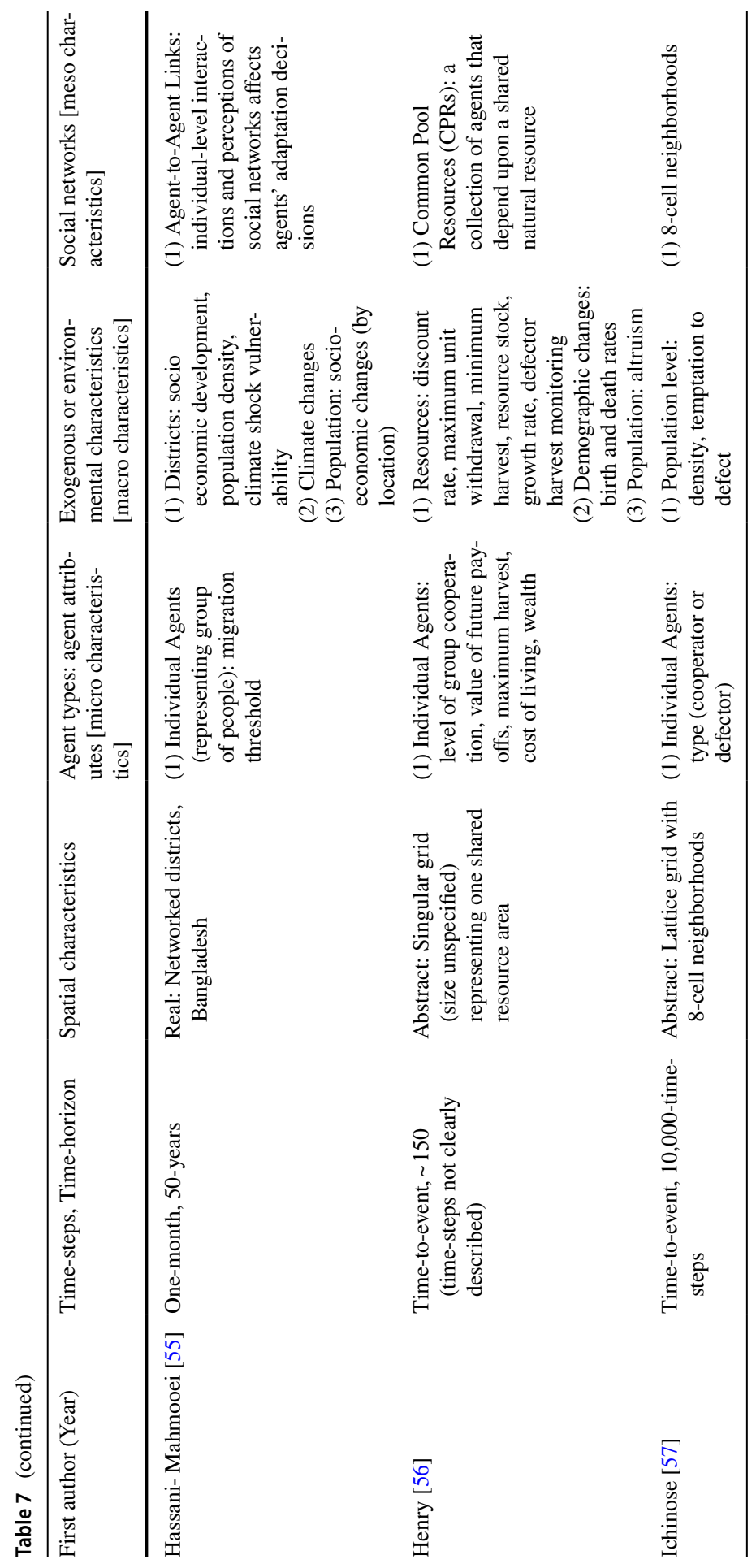




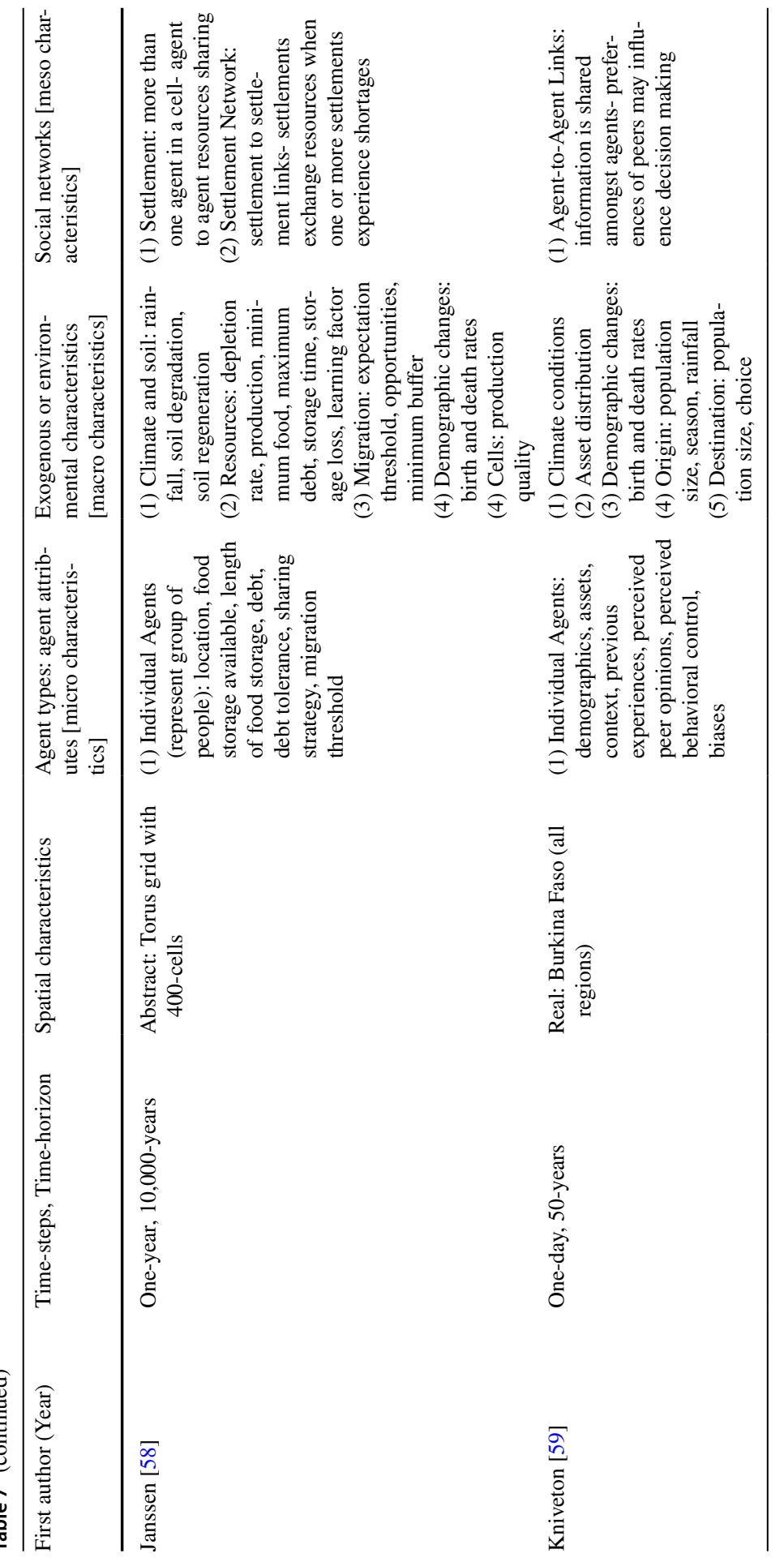




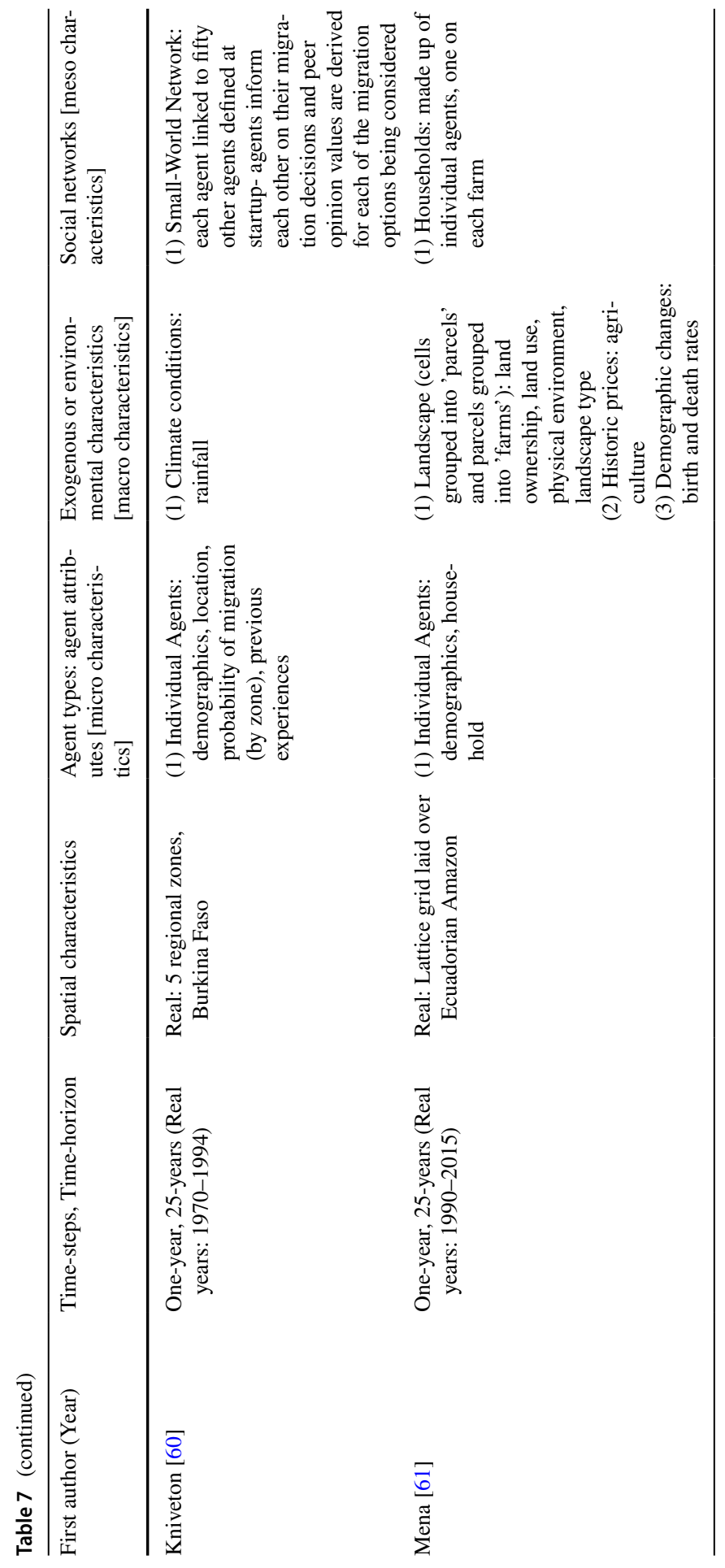




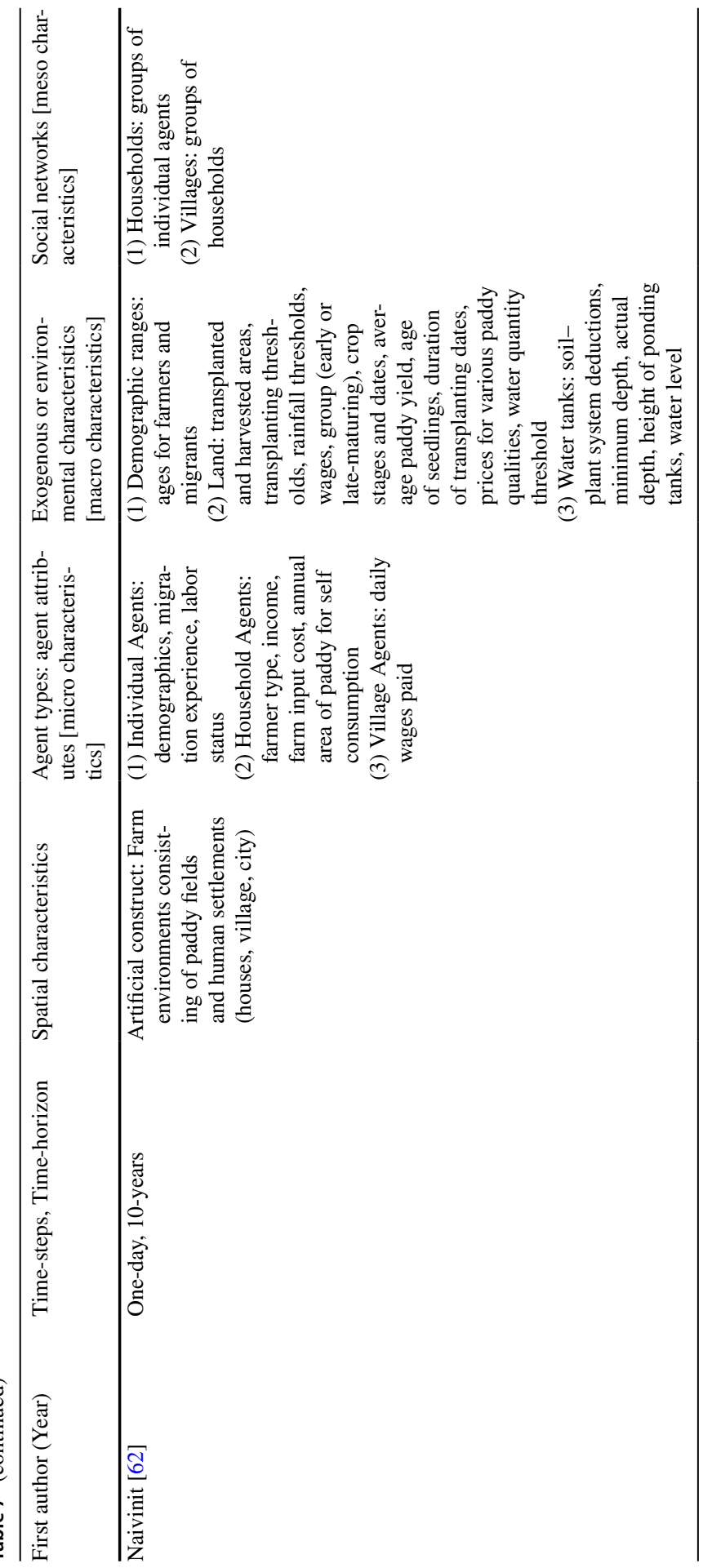




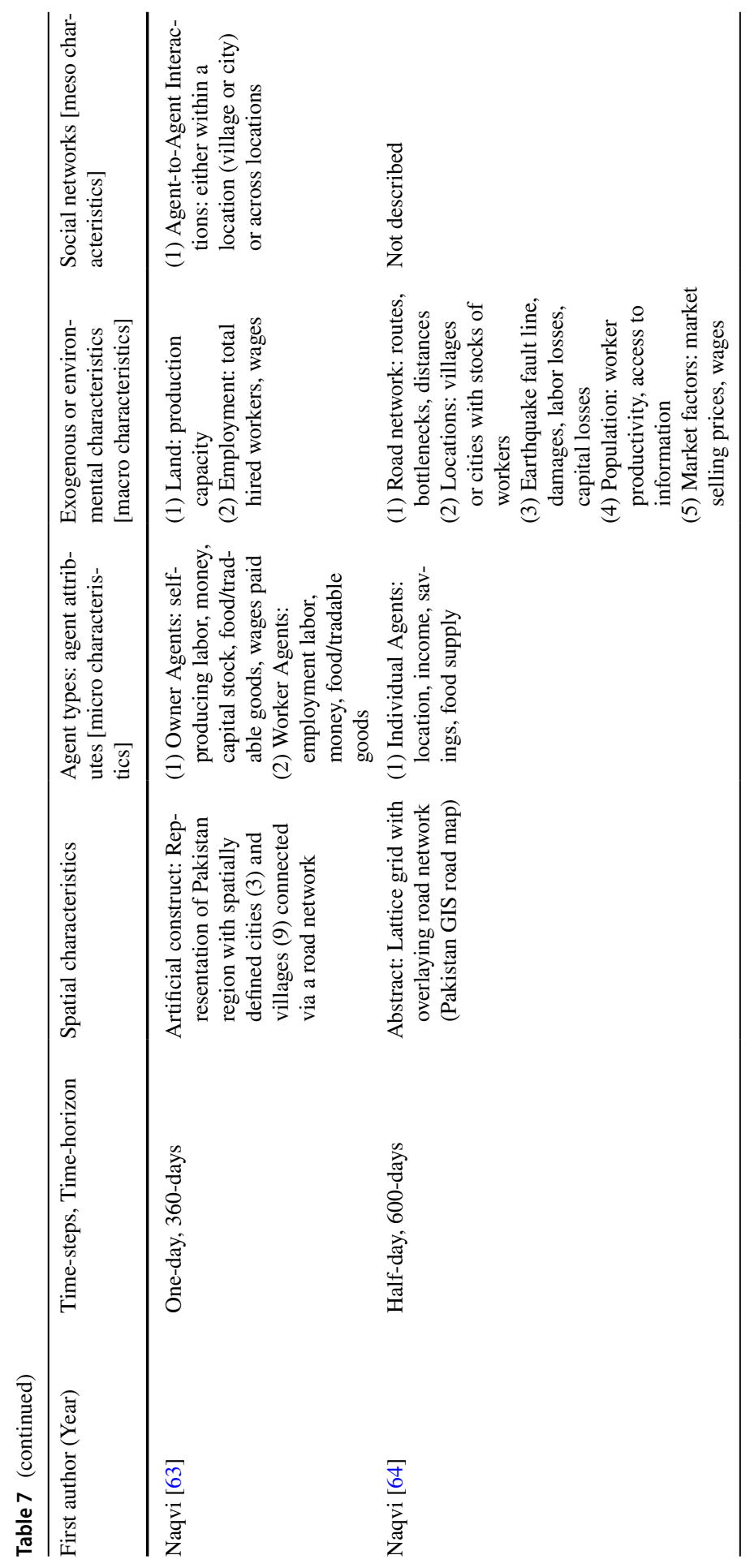




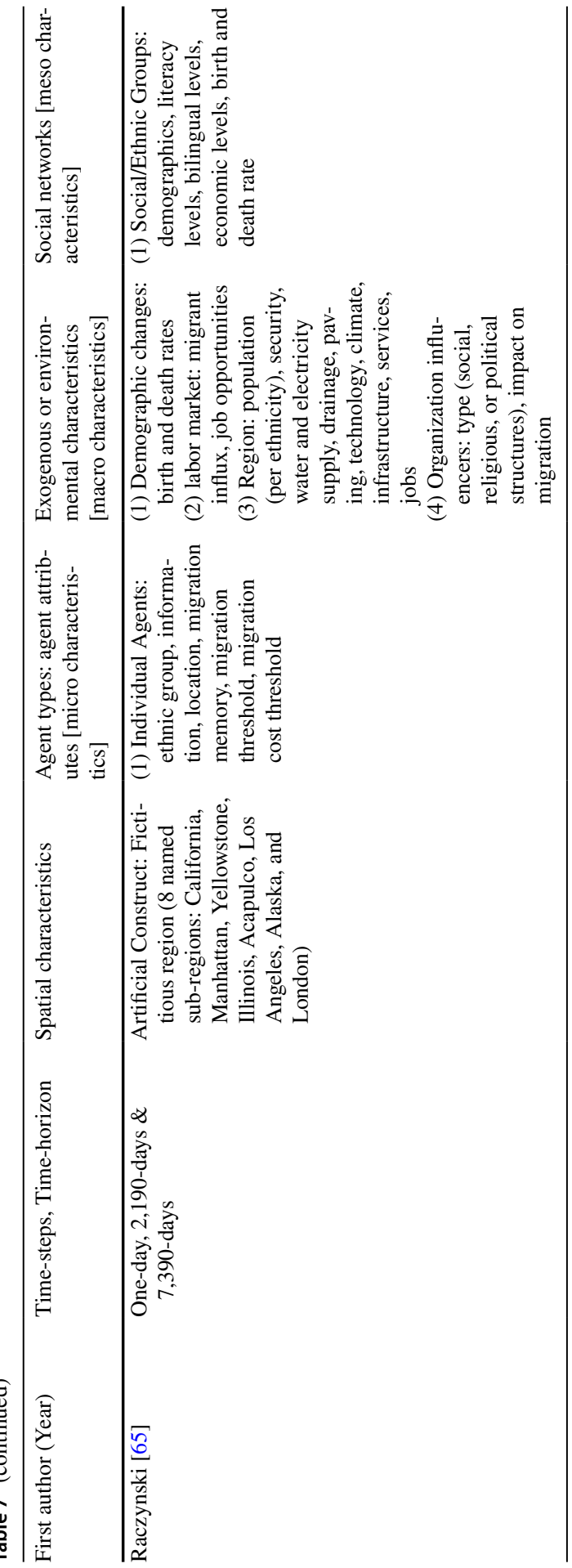




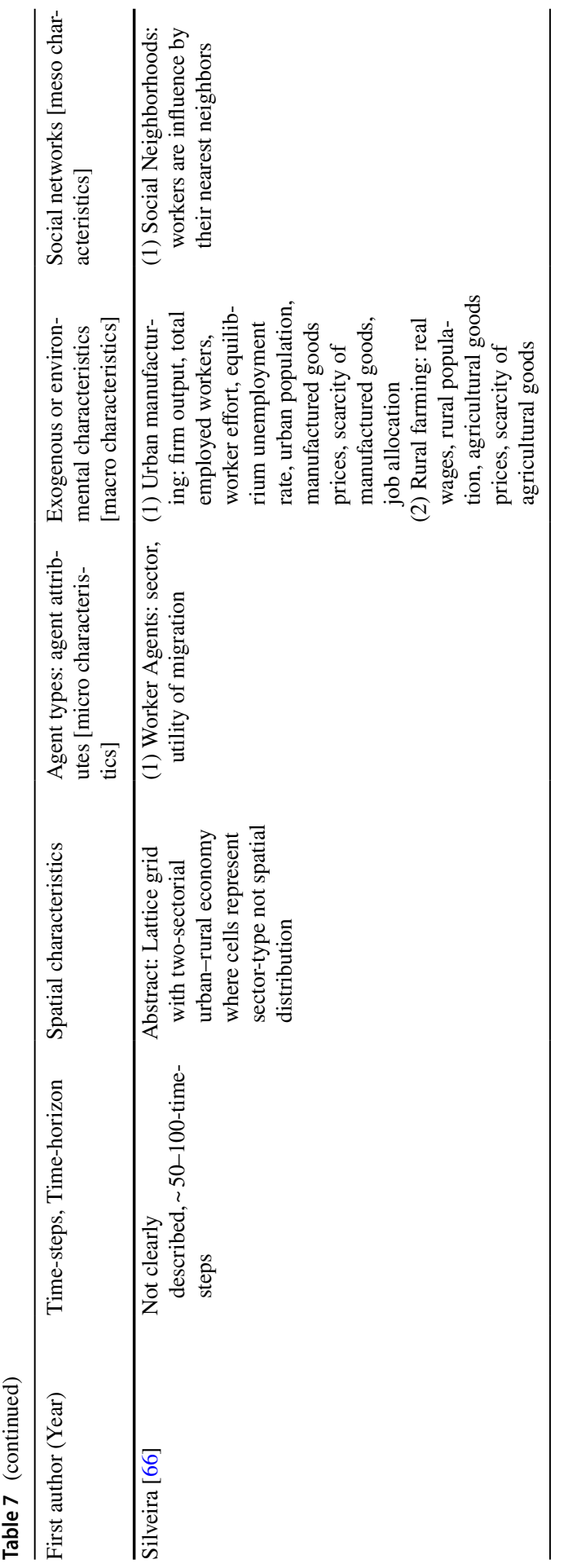




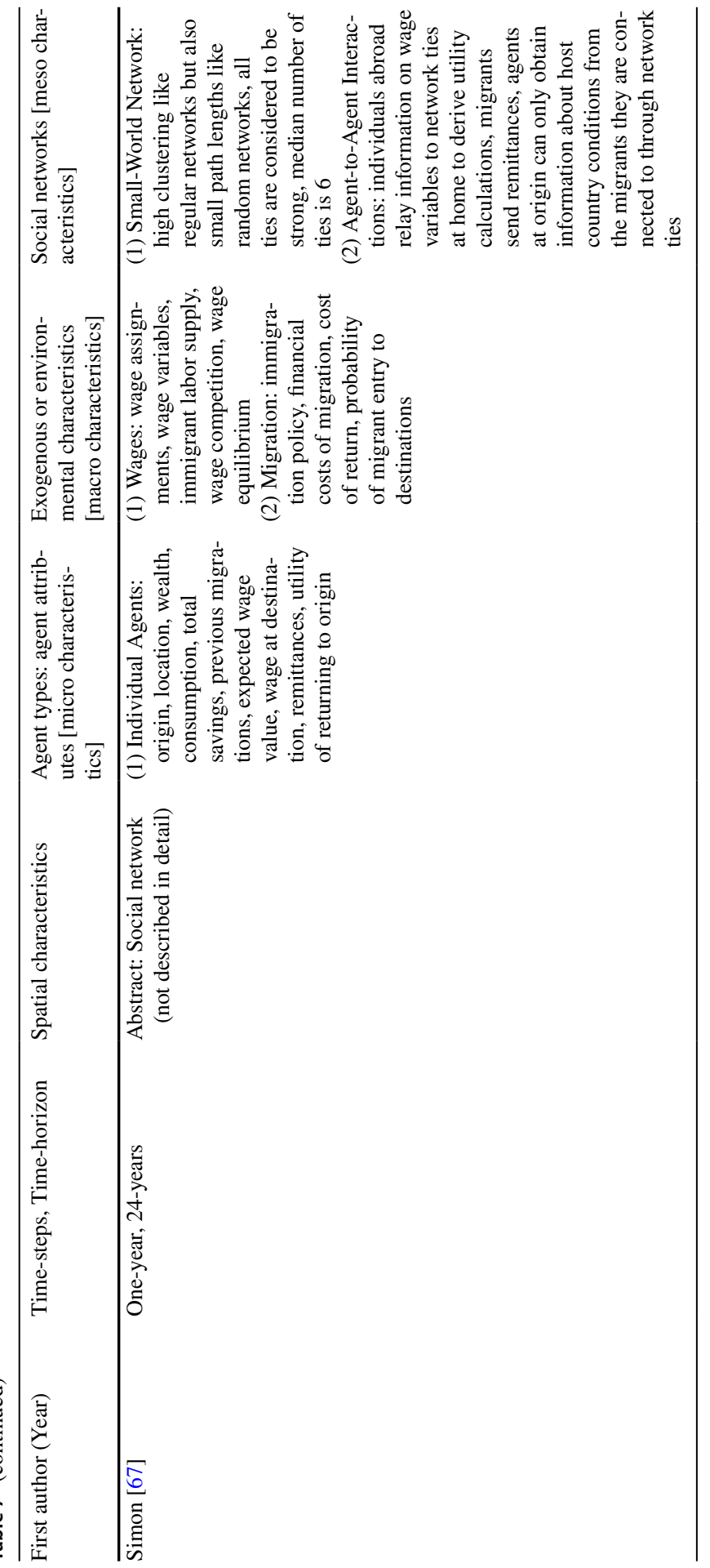




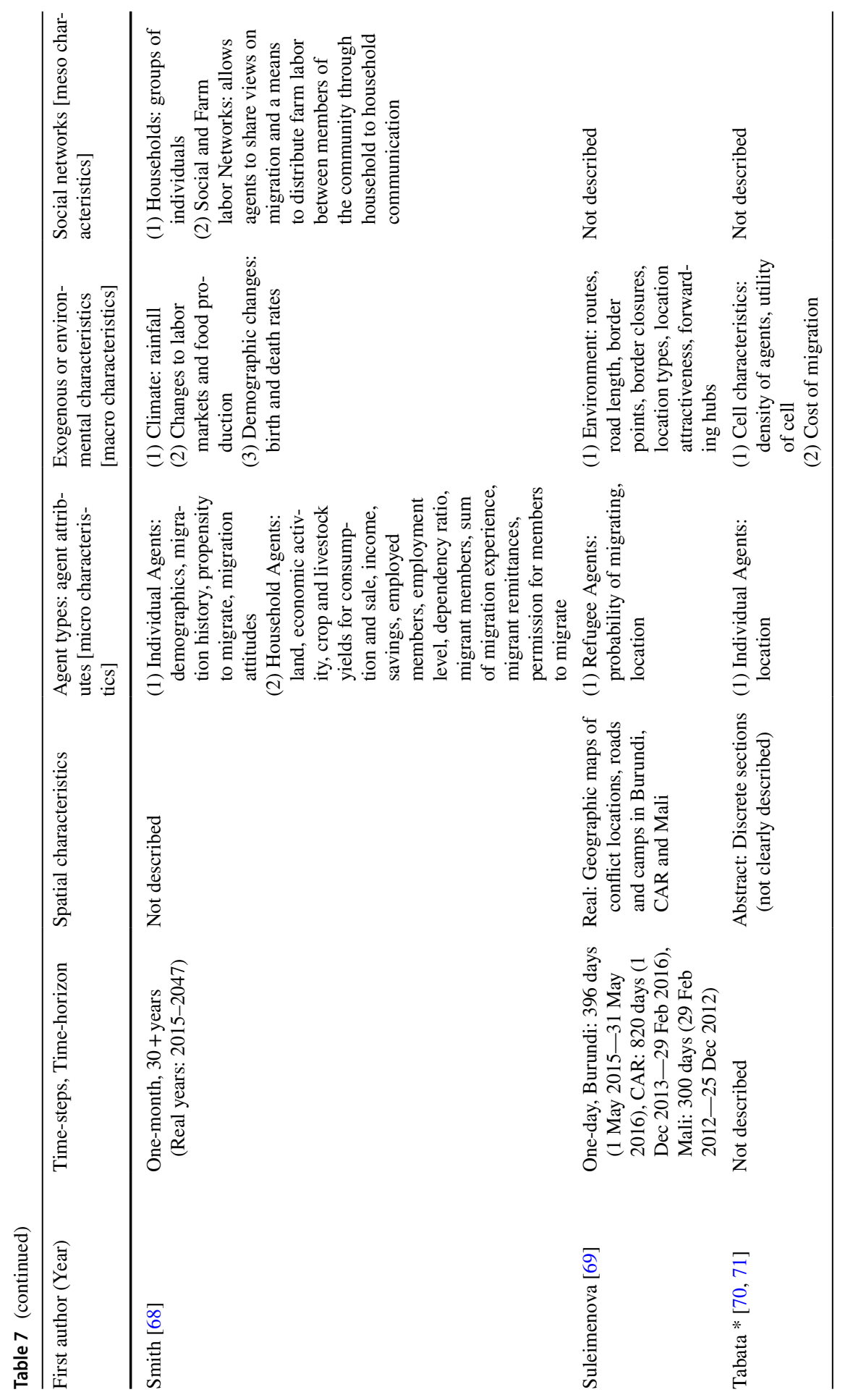




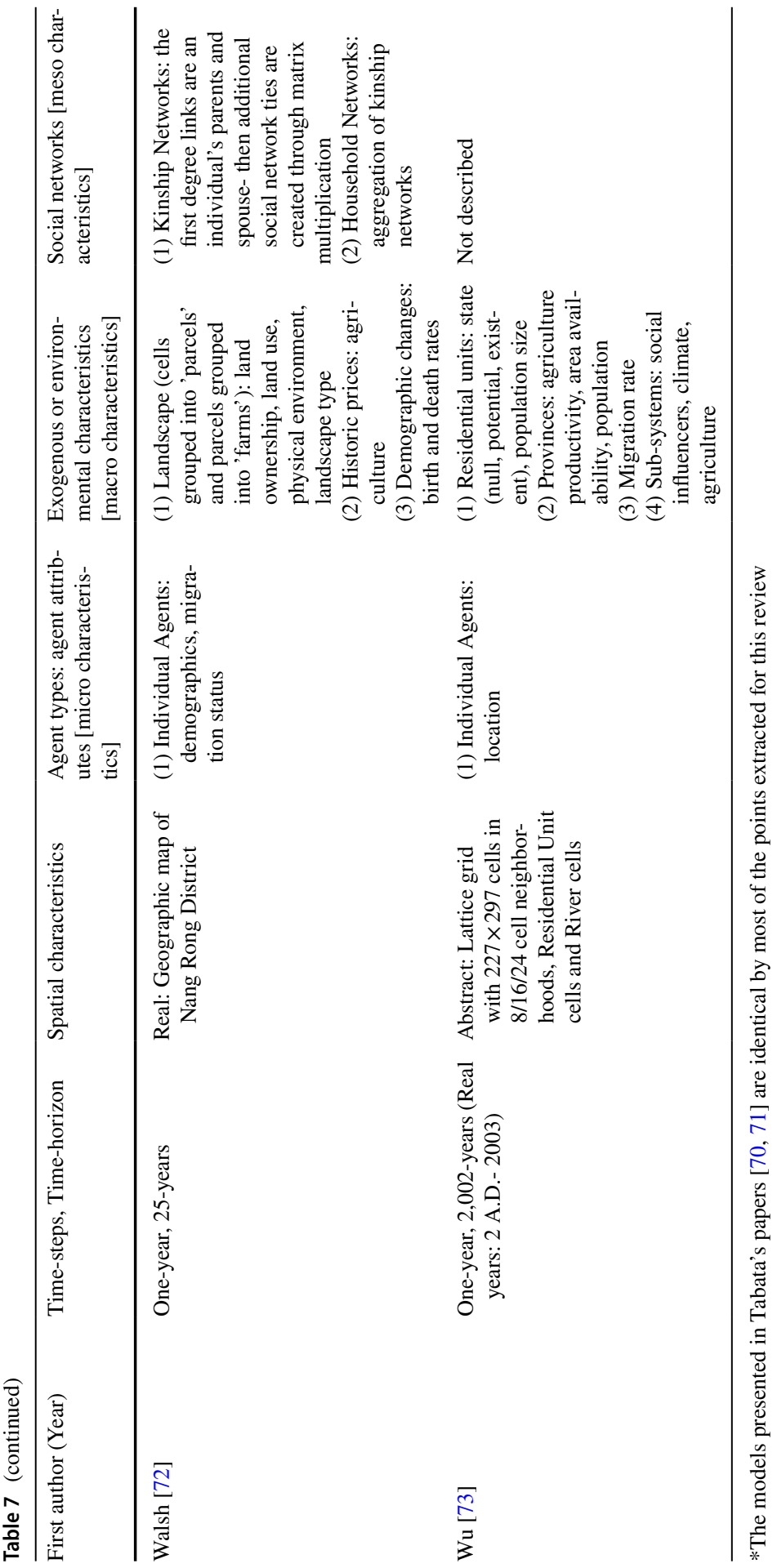


Spatial characteristics are often an important design decision in ABM and the spatial representation depends on the research aims and data available (See Table 7). Some used abstract spatial representations, such as grids or networks $(n=14)$, some used artificial constructs, such as a refugee camp setting or generic farm landscapes $(n=4)$, and finally some used real spaces often mapped using GIS data $(n=9)$. One study did not describe the spatial elements in enough detail to classify.

Along with time and space, deciding the agents and respective attributes is key to ABM development. Often the aim is to make models as complex as they need to be but no more, often referred to in the ABM modeling community as Occam's Razor principle or the KISS principal ('Keep It Simple, Stupid') [79]. This can be a difficult aim to achieve when deciding how many distinct agent types are needed and what attributes are required for the various decision-making processes and interactions included in the model processes. Most of the models included in this review only have one type of agent $(n=15)$, sometimes named as migrants, or workers. Some models include a second or third type of agent, such as employers, land owners, leaders, or government agents and still some models used secondary groupings of individual agents to represent households or villages with their own unique attributes (See Table 7). Most often, these agents have a range of heterogeneous attributes, such as demographics, socio-economics, propensity to migrate or act, assets, etc., but in three of the studies, agents were only characterised and distinguished by location and not by any additional attributes $[70,71,73]$.

The models also described various exogenous or environmental characteristics that impacted on the parameters of the model or the agent's behavior within the model. Some examples included, location-specific variables, such as population levels, services available, distance to travel, wage differentials, housing capacity at destinations, etc. (See Table 7). A common exogenous factor was weather or climate $(n=8)$, often varying across scenarios or time-steps. Finally, some demographic and epidemiological rates were defined at the population level, such as birth rates, death rates, migration rates, sickness rates, etc. These exogenous or environmental factors are not within the individual agent's control and reflect the larger system(s) that these migration- or work-related decisions are taking place within. Another broader model characteristic that impacts individual agent actions are the social networks that agents can be grouped into, such as households, villages, intra-group networks, and non-spatial social networks. A majority $(n=21)$ of the studies used some type of agent grouping or network in addition to individual agents (See Table 7).

4. Model process

The model process takes on a very unique form for every ABM. It would be impossible to fully synthesize the initialization, model steps, decision rules, agent actions and scenario descriptions across all 28 studies in a meaningful way. We recommend the reader inspect the columns of Table 8 to get an overview of the design of the model process as it is simulated in each model. Most of the models 


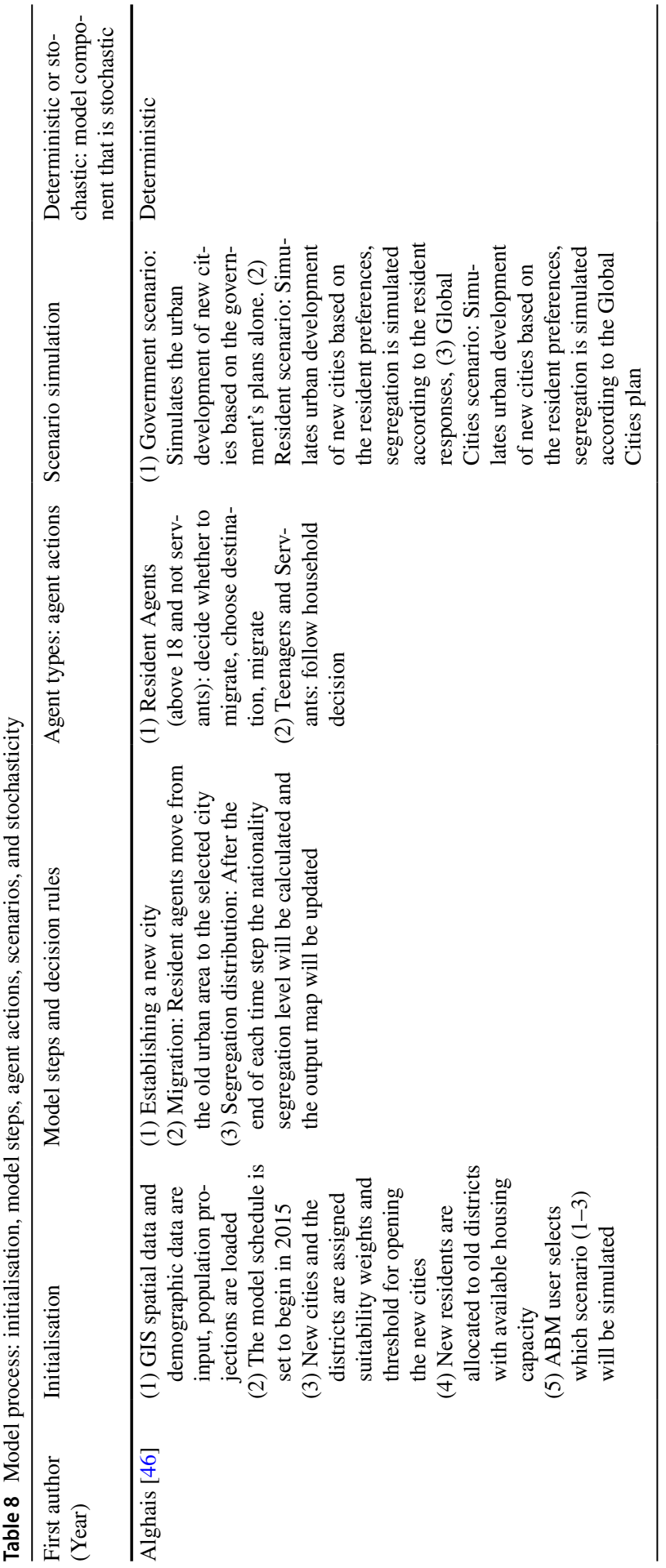




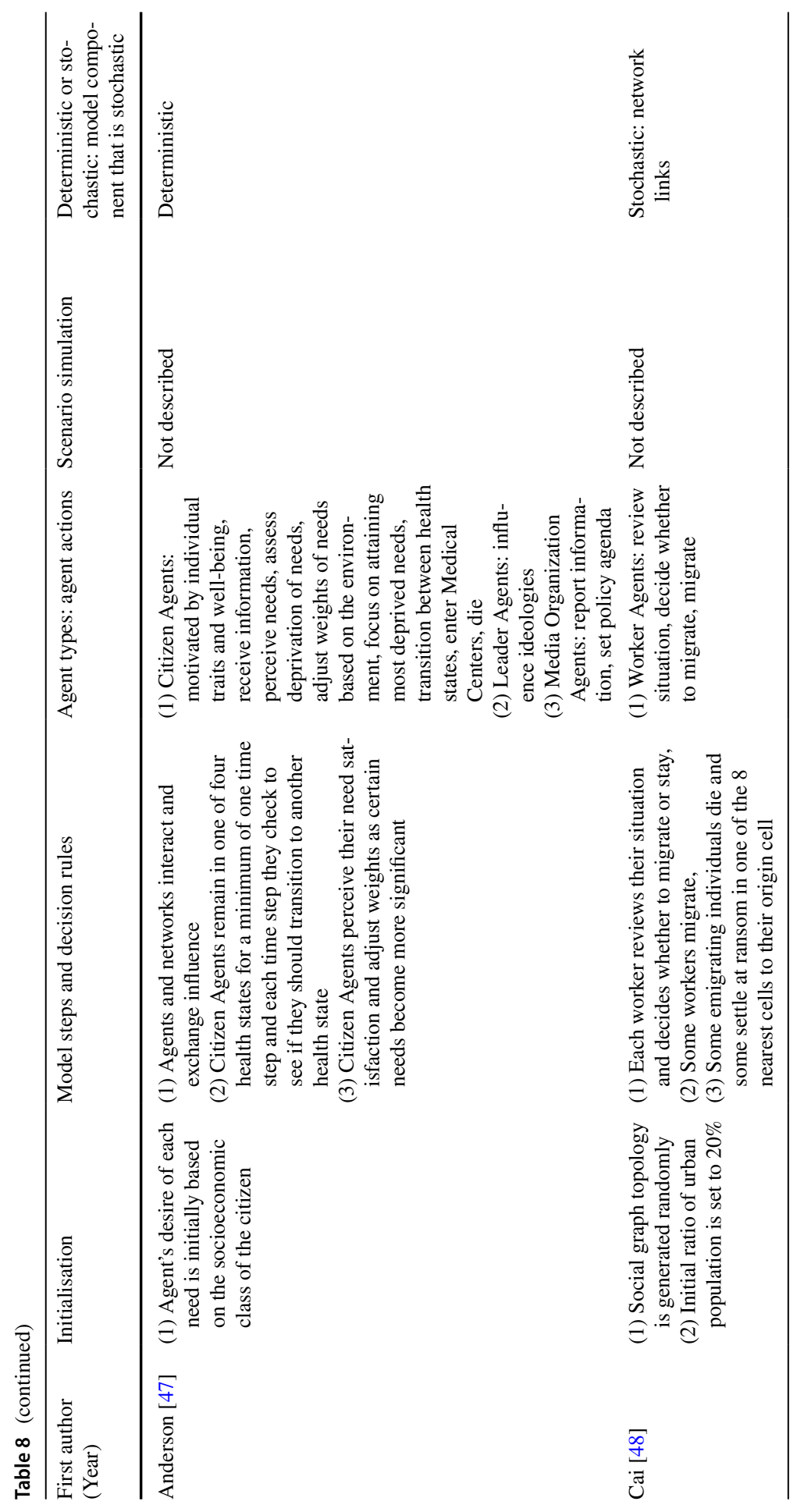




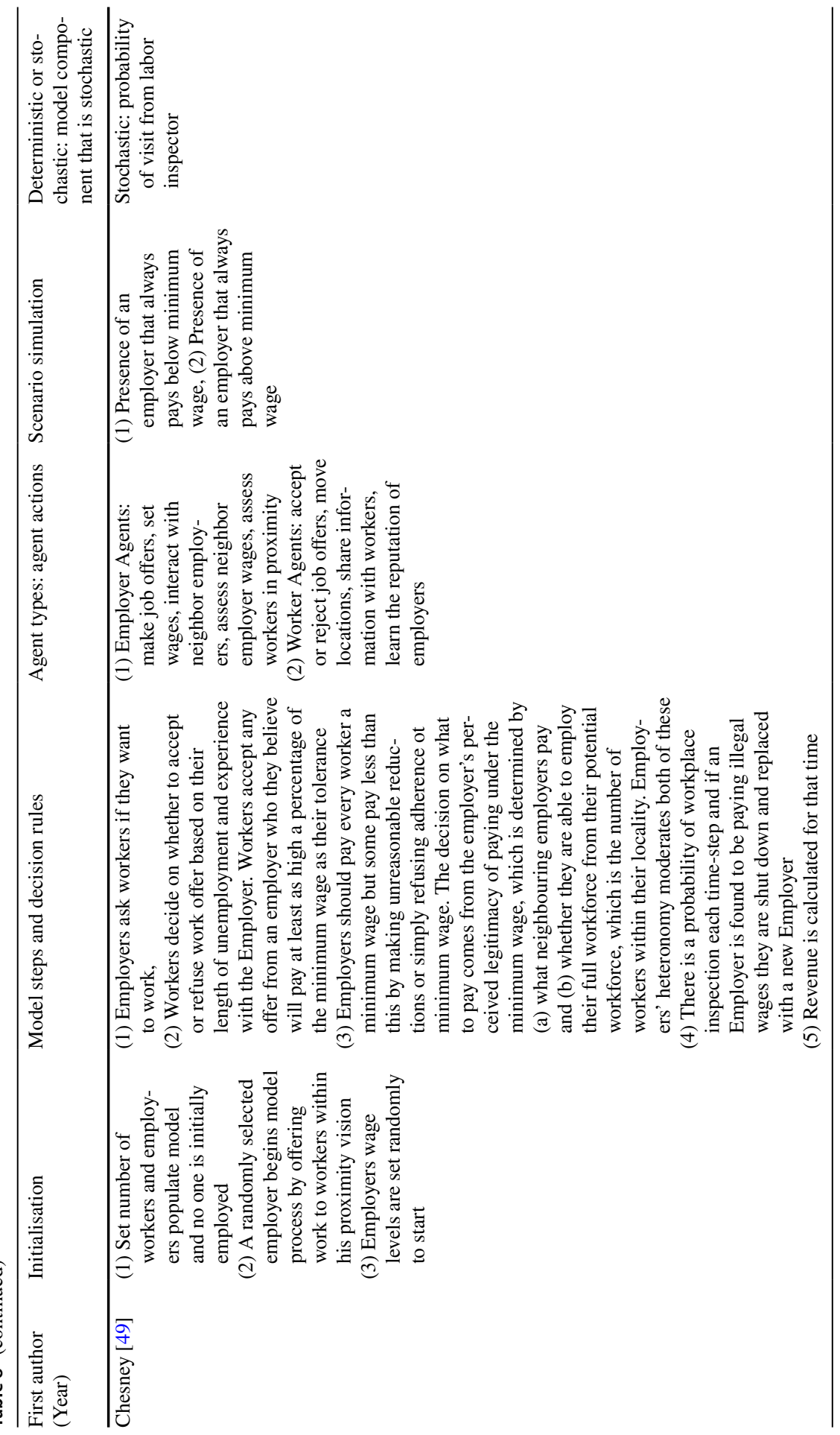




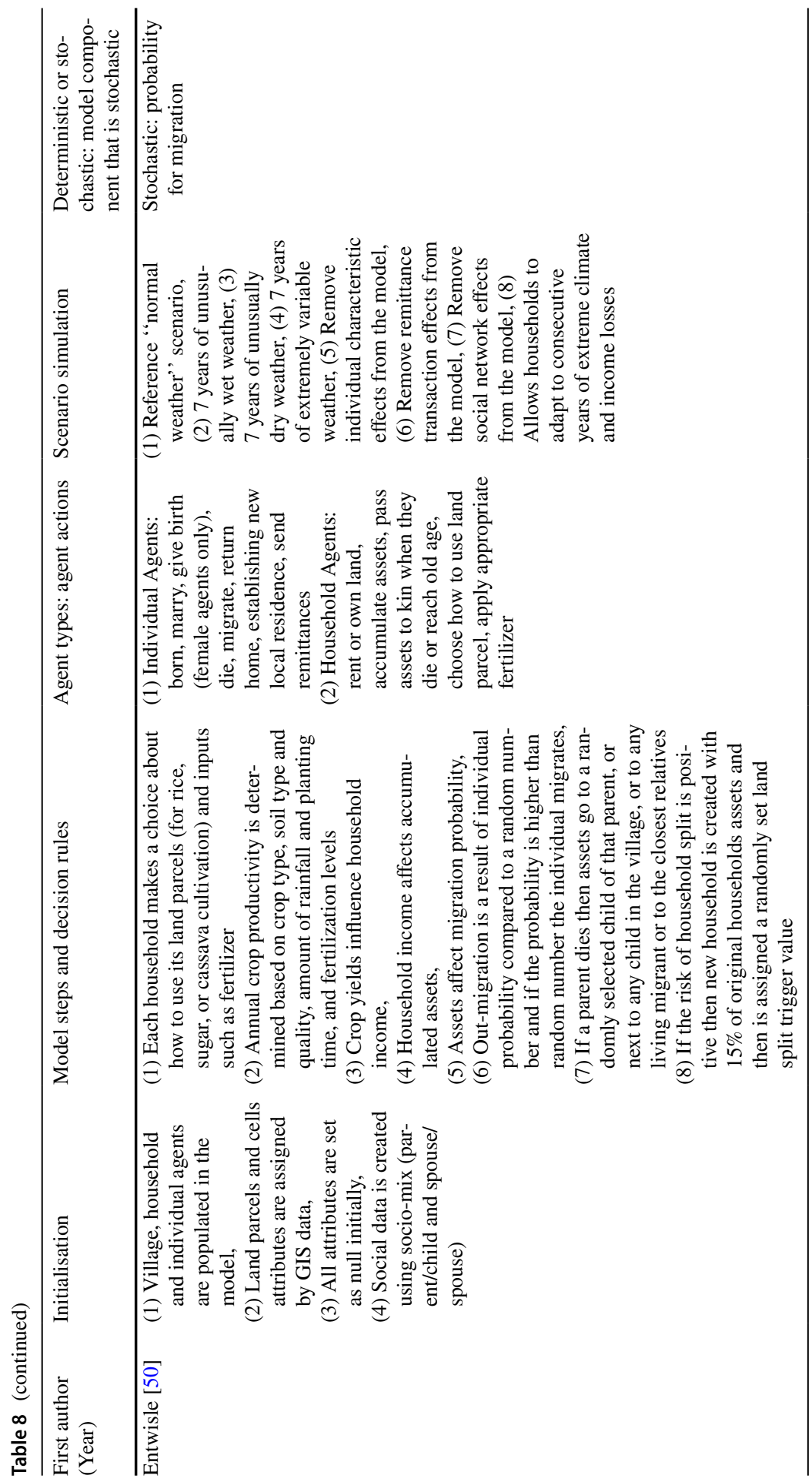




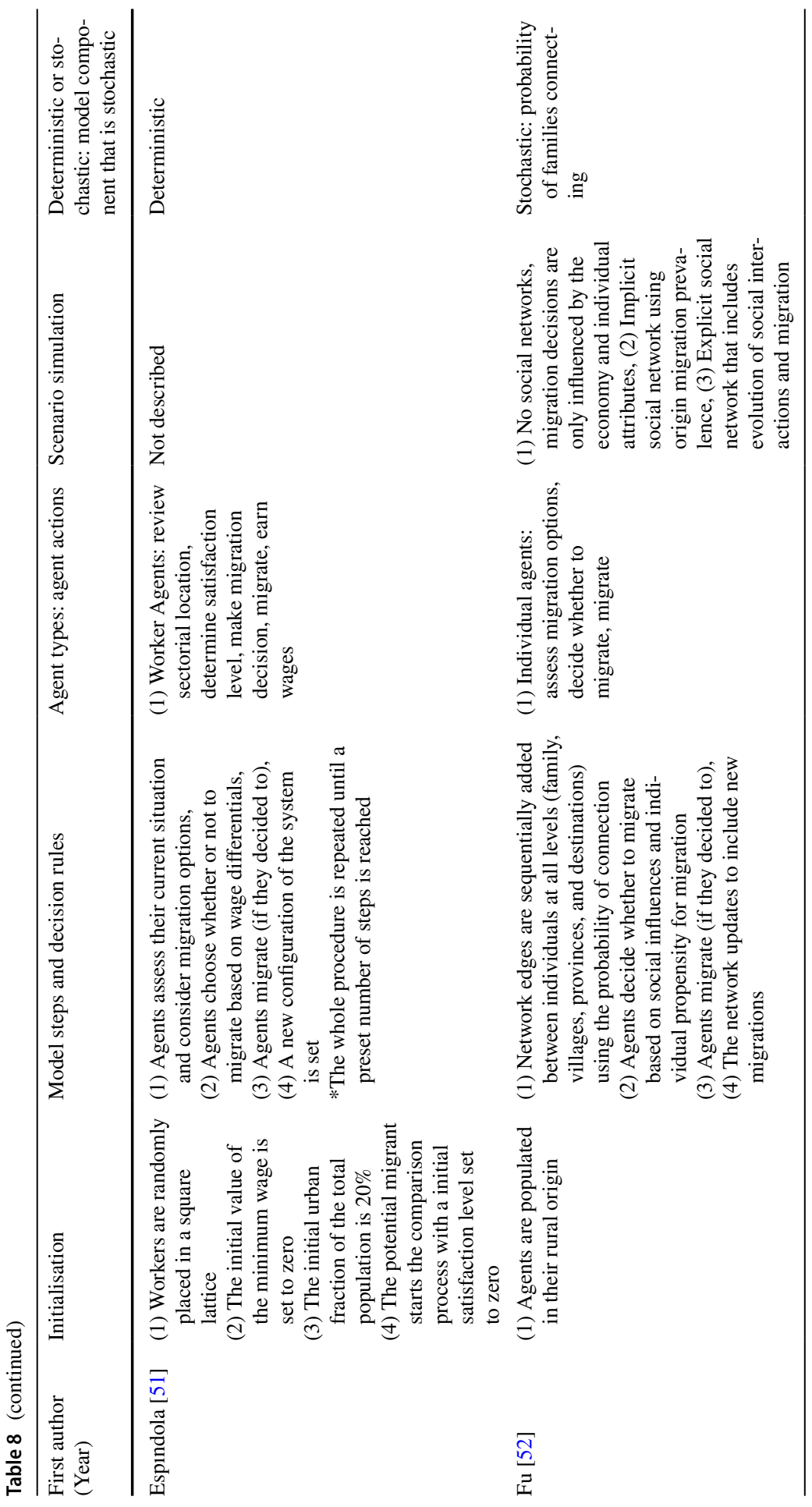




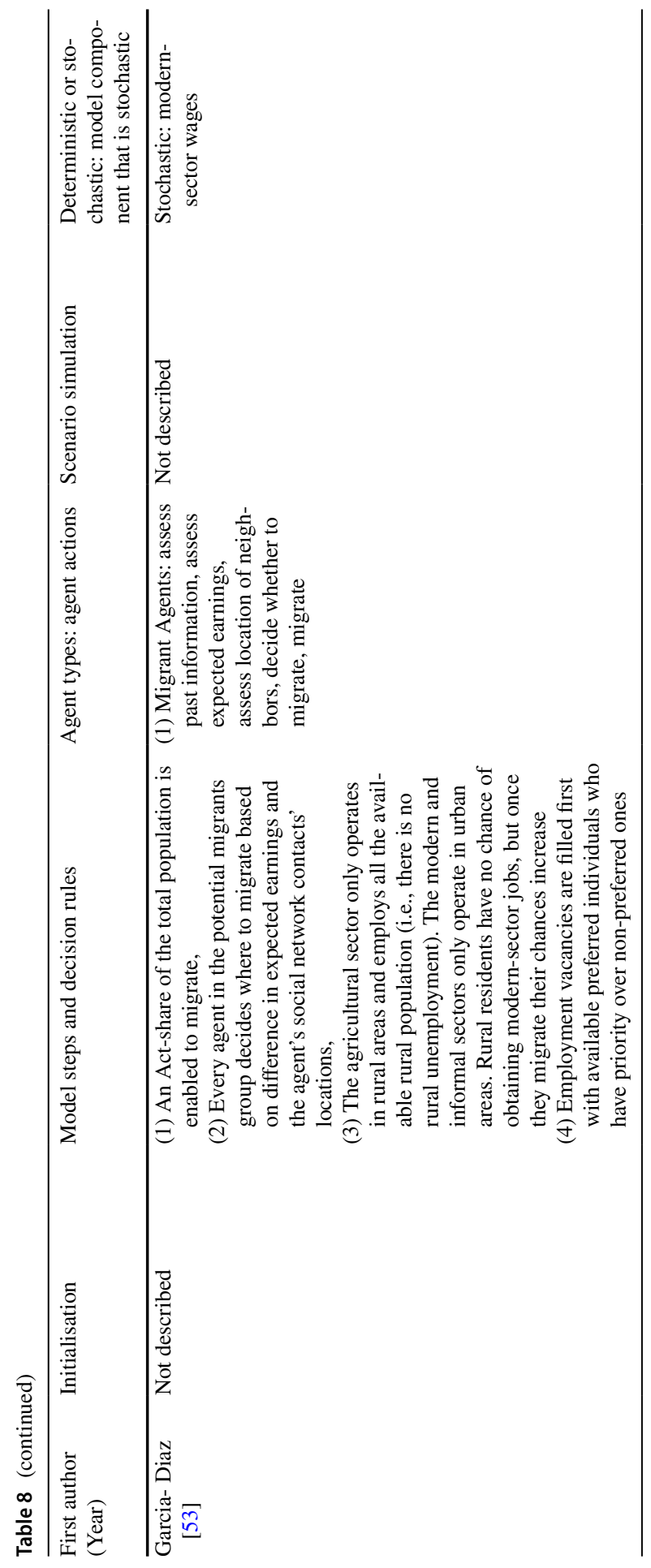




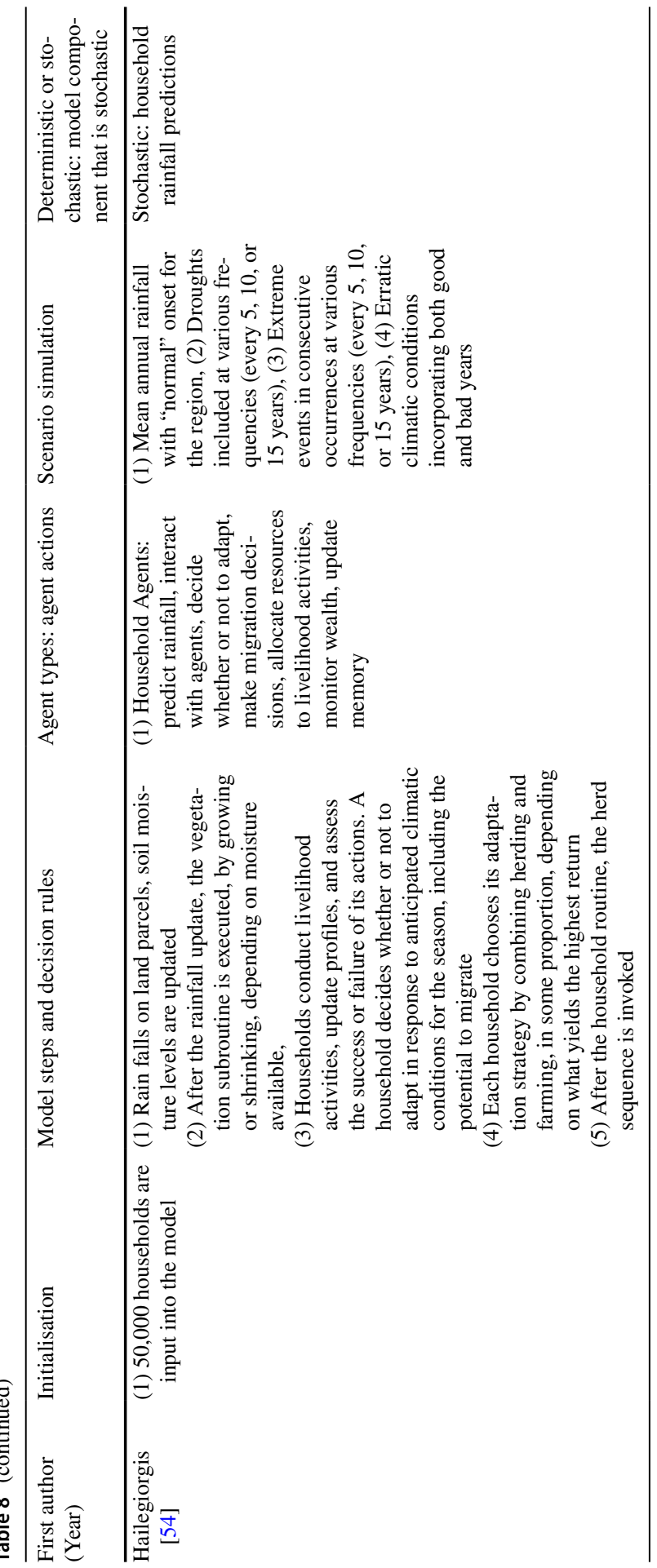




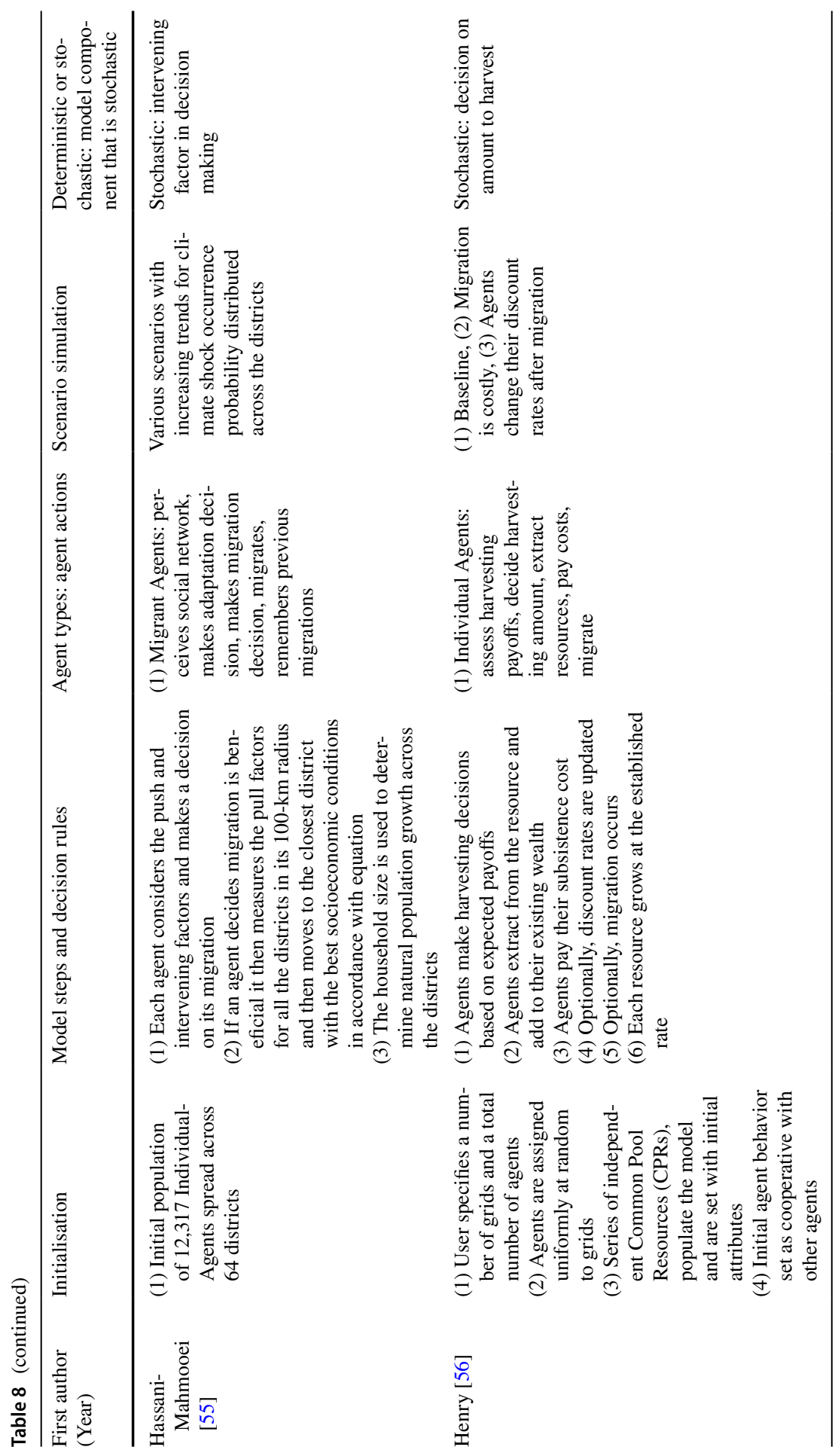




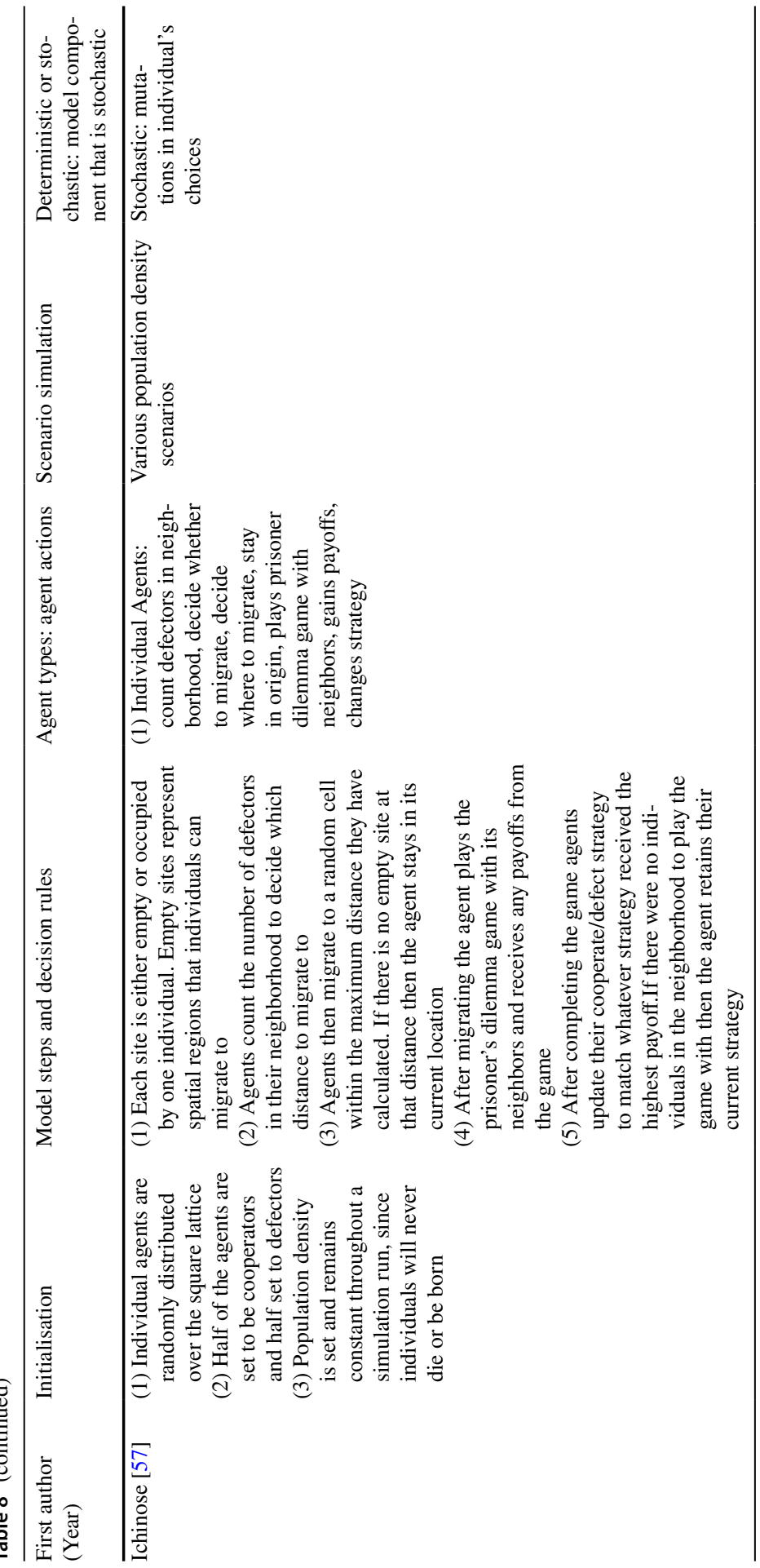




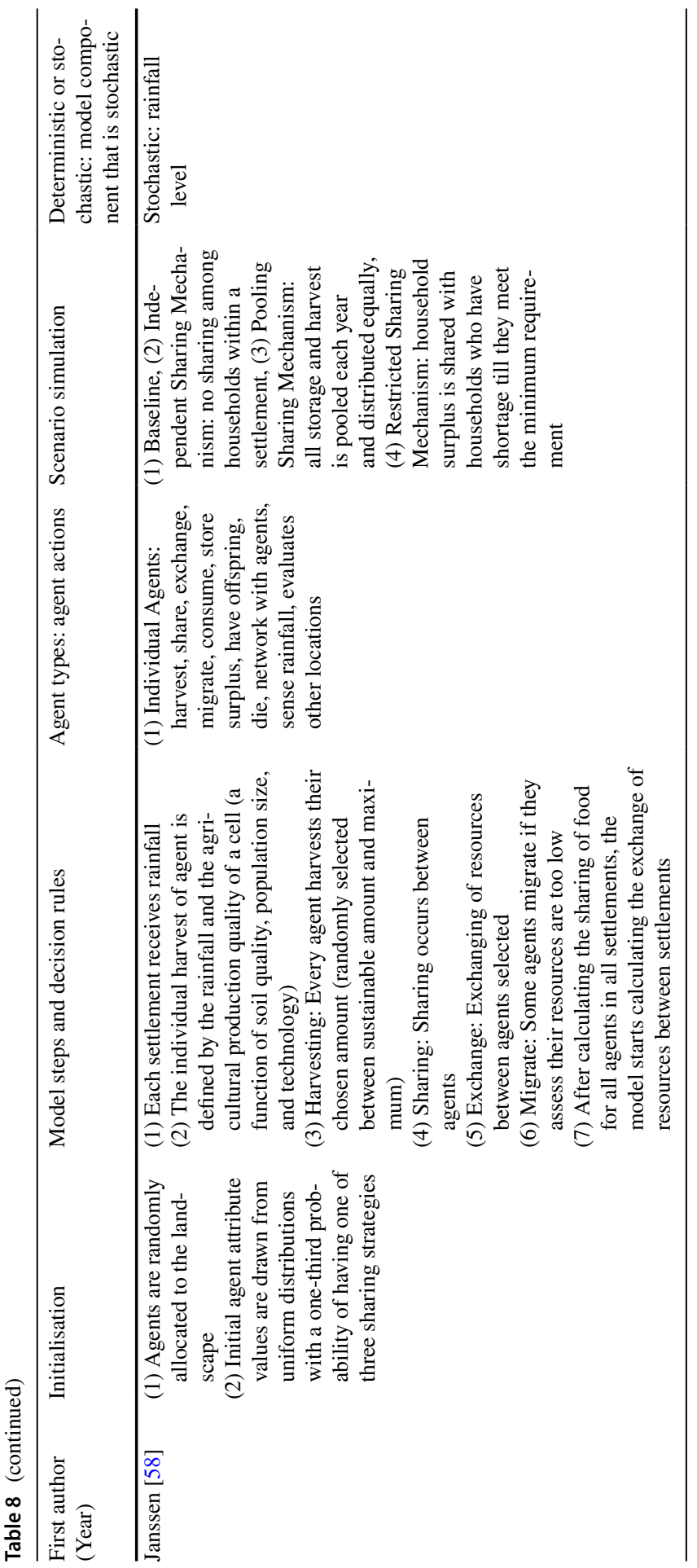




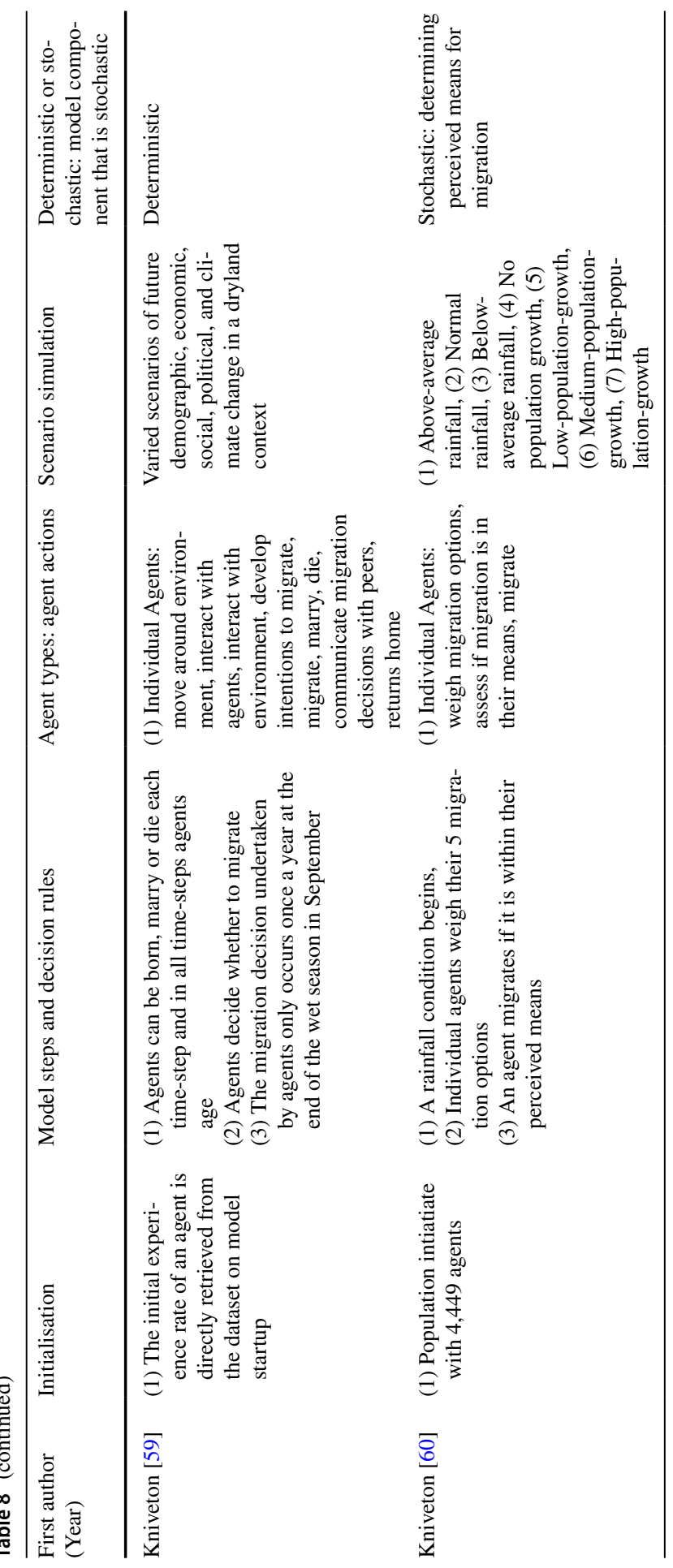




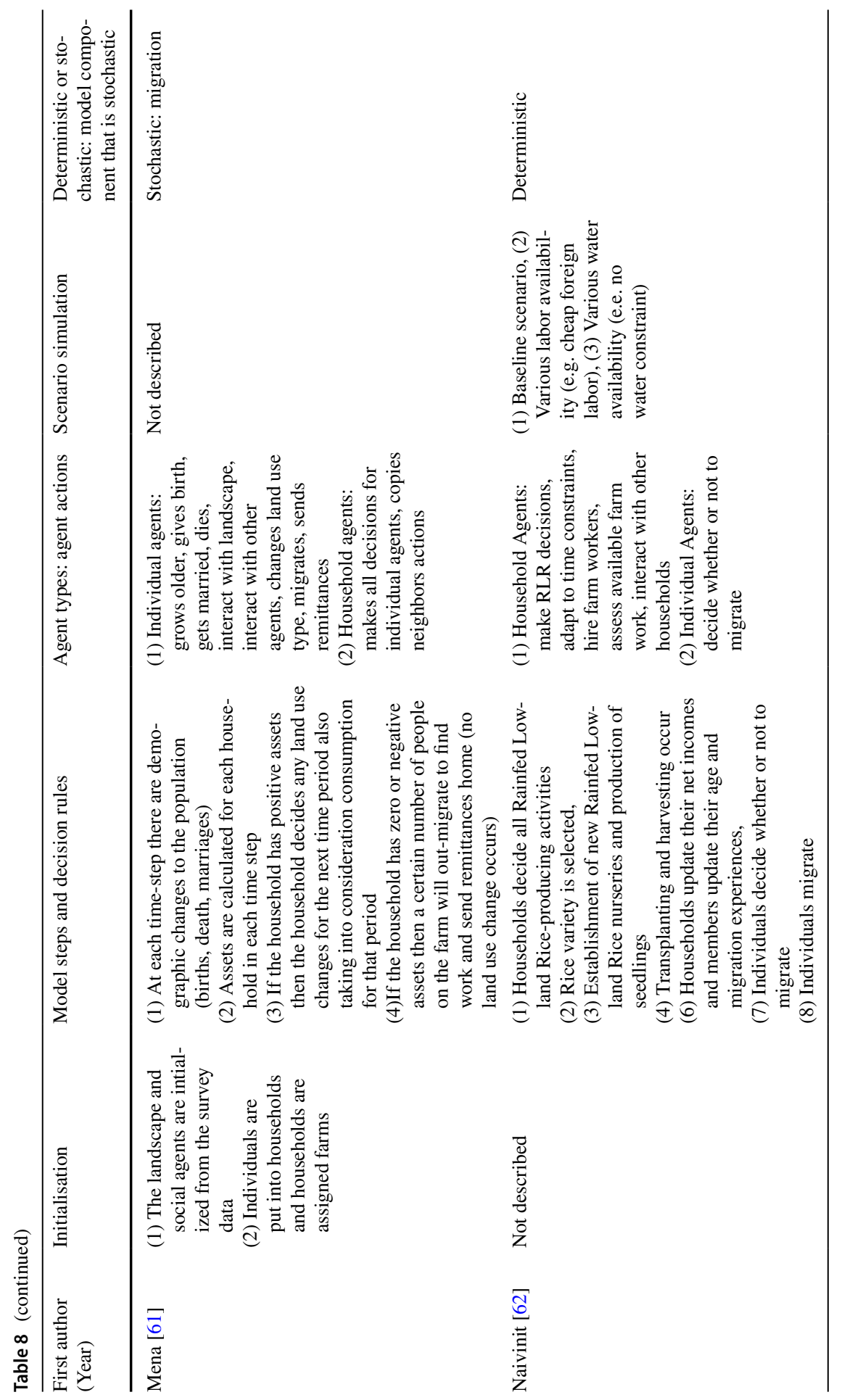




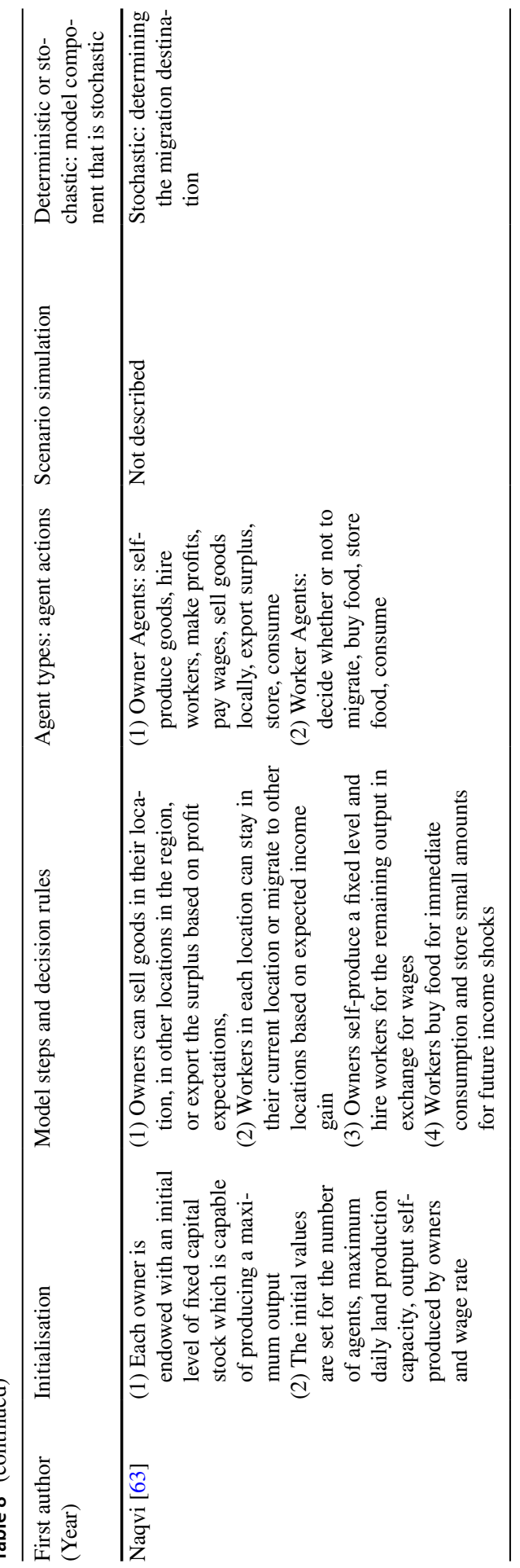




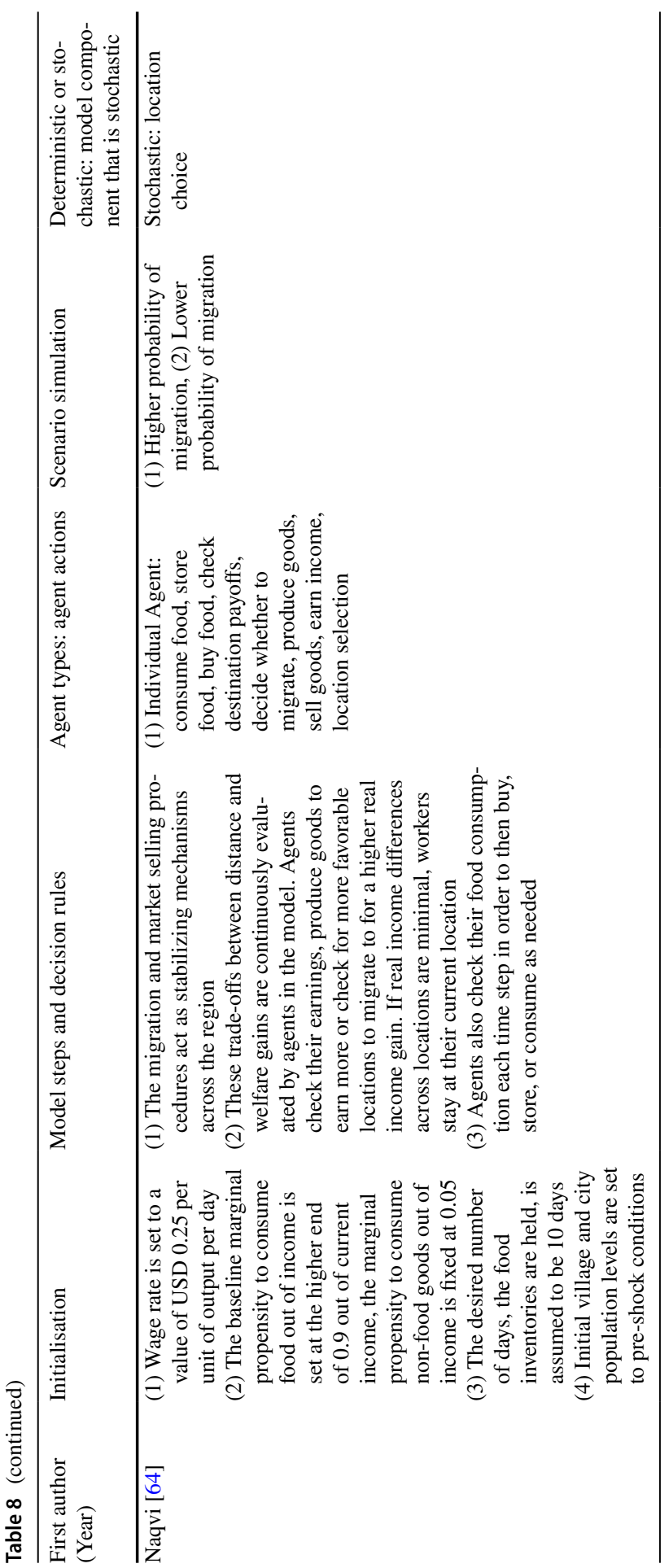




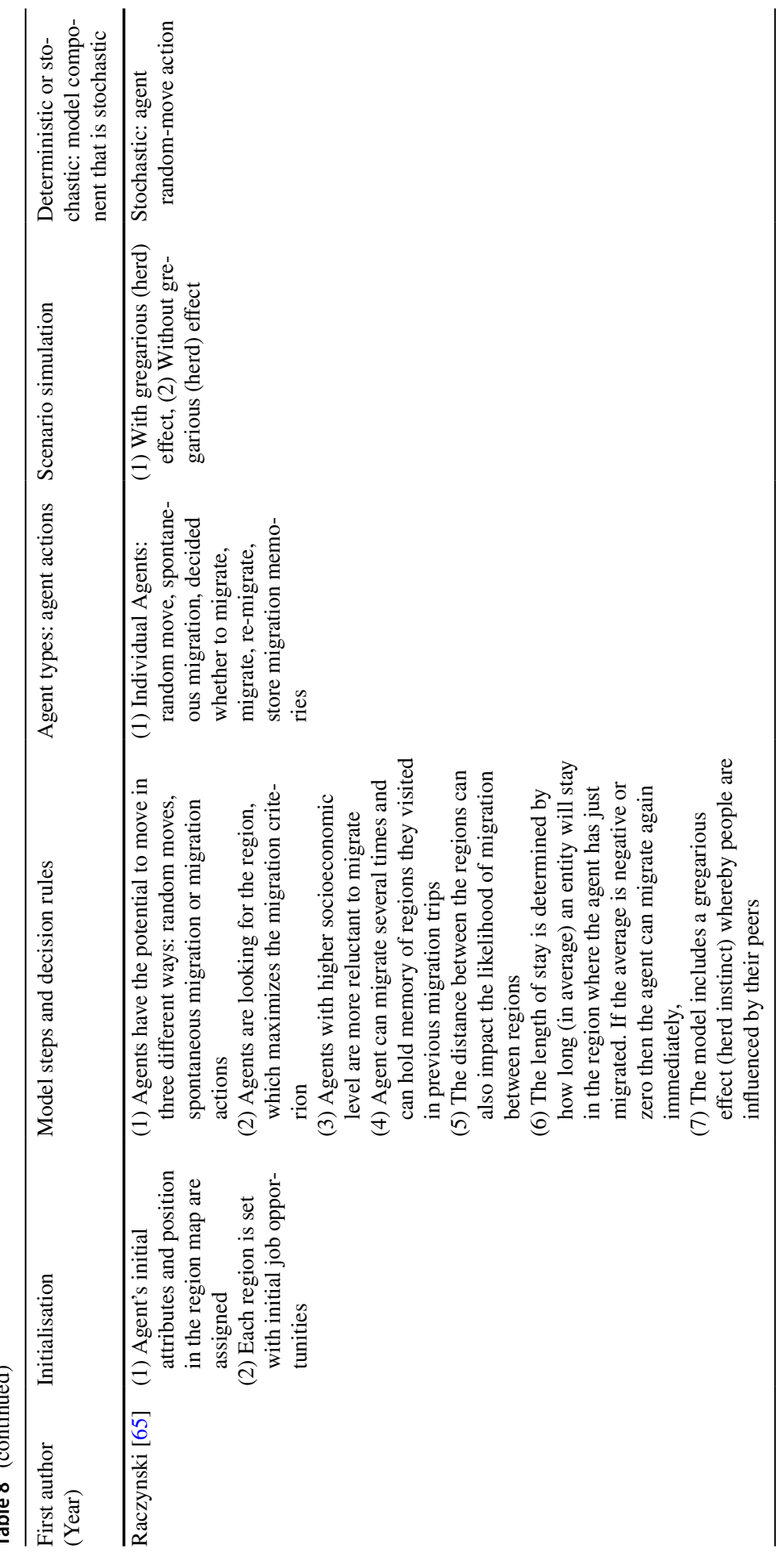




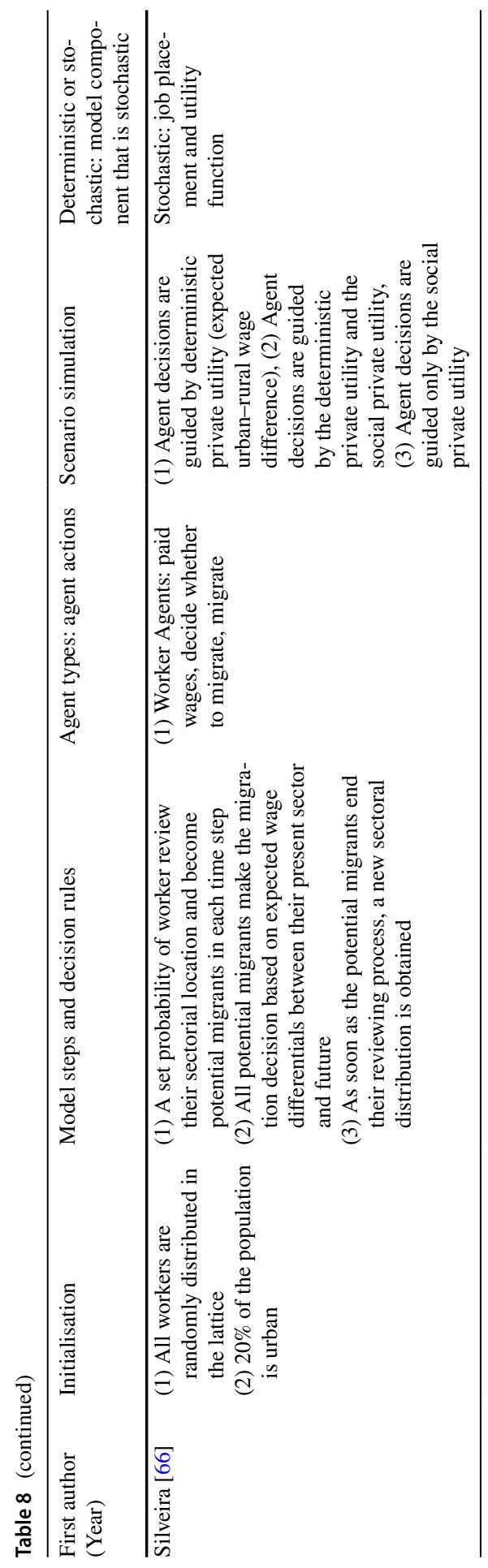




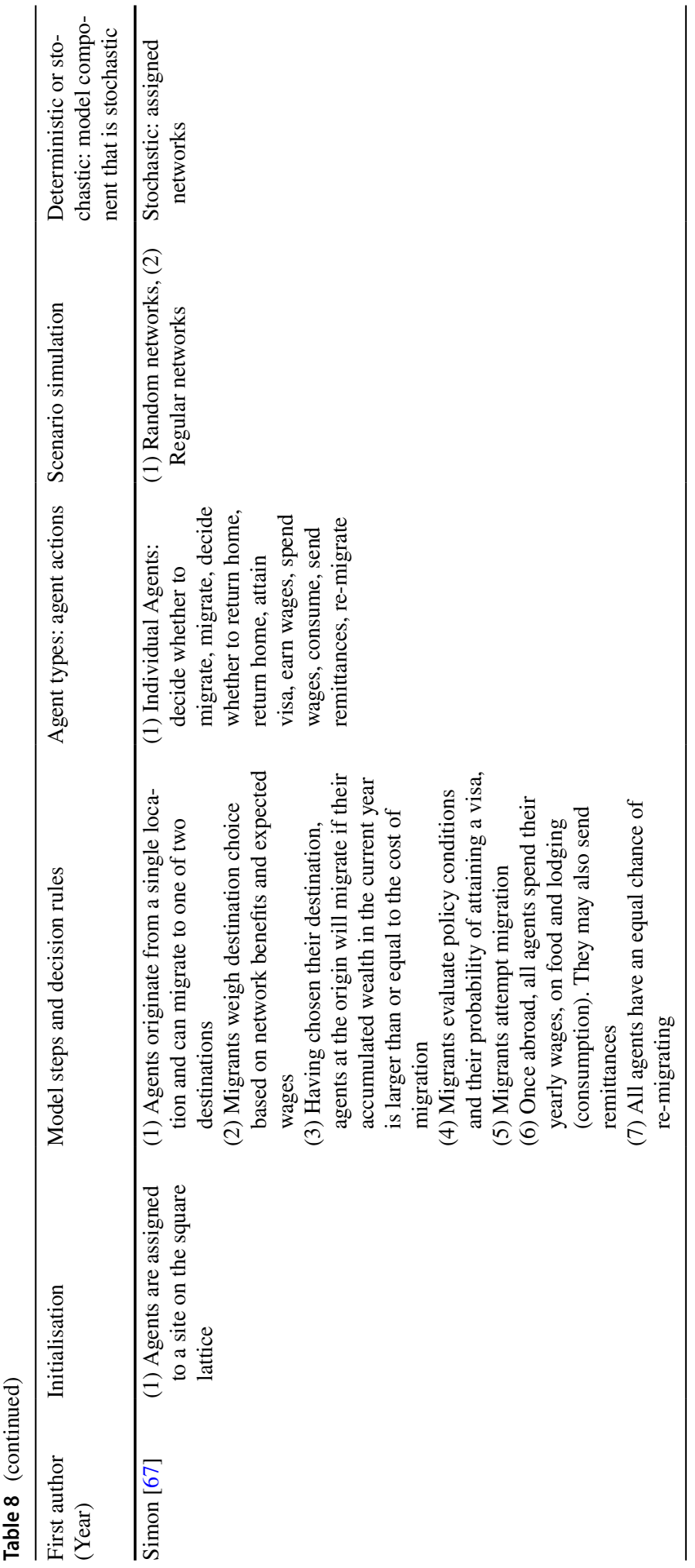




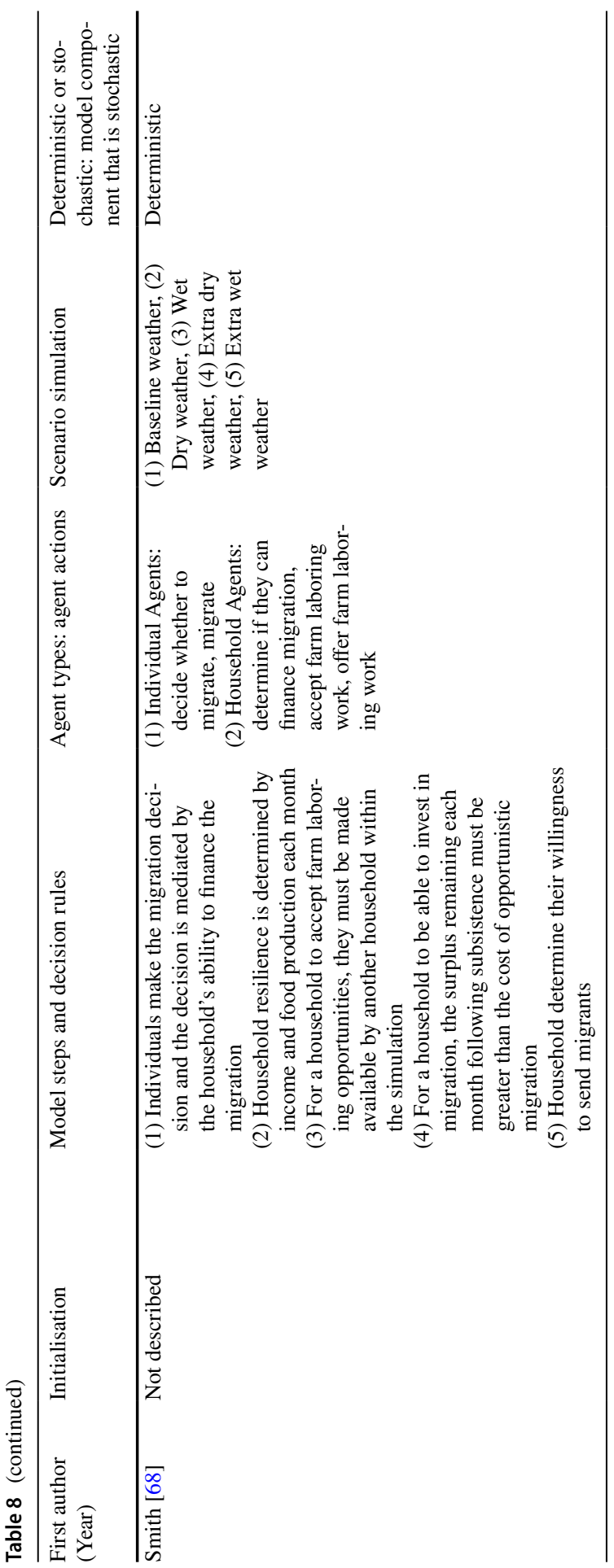




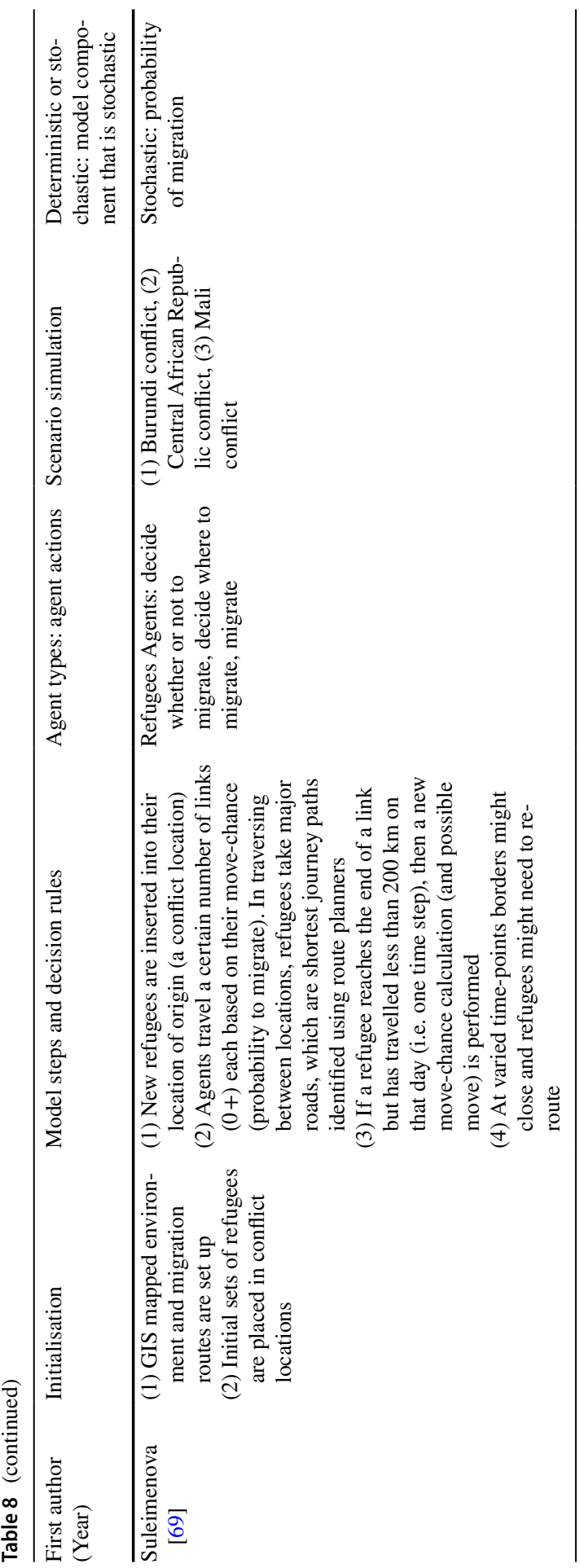




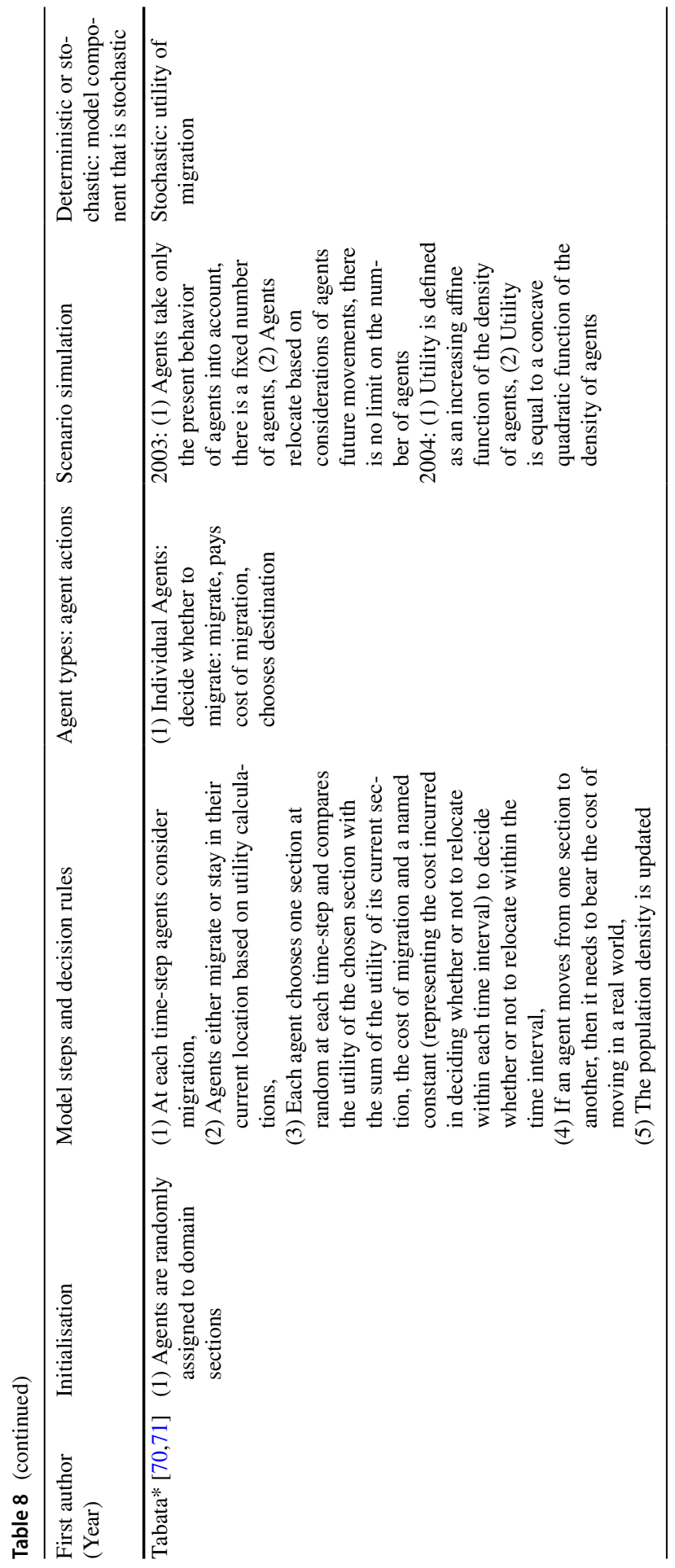




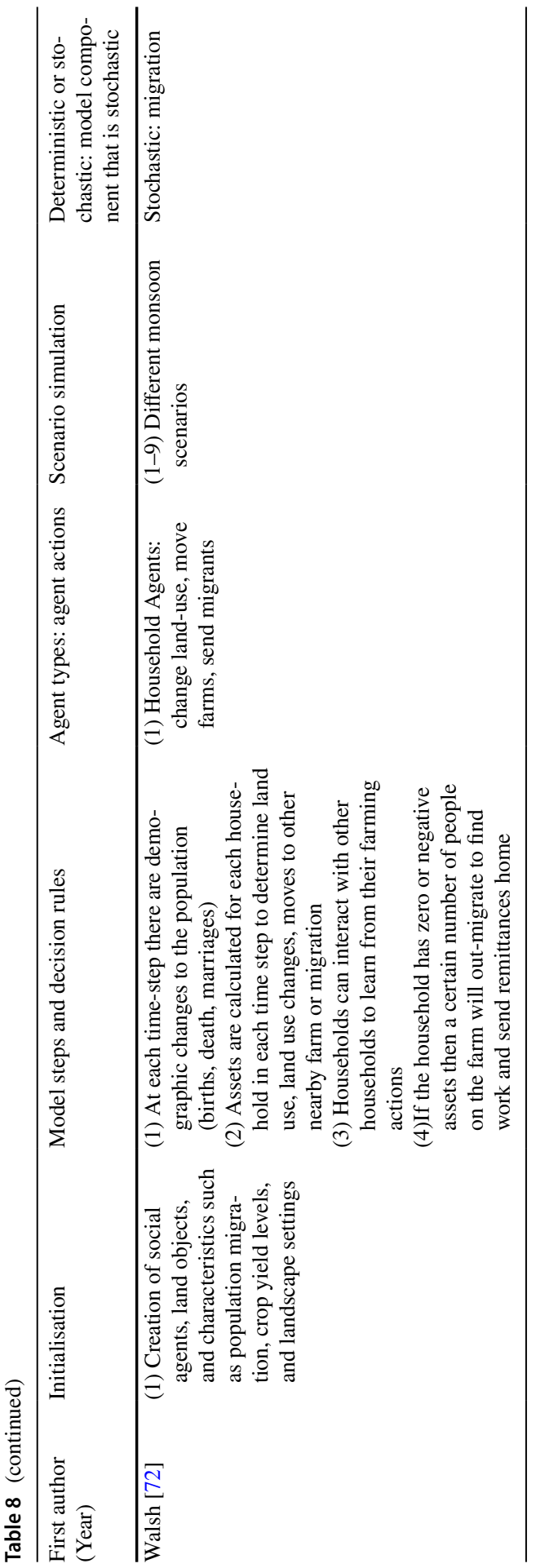




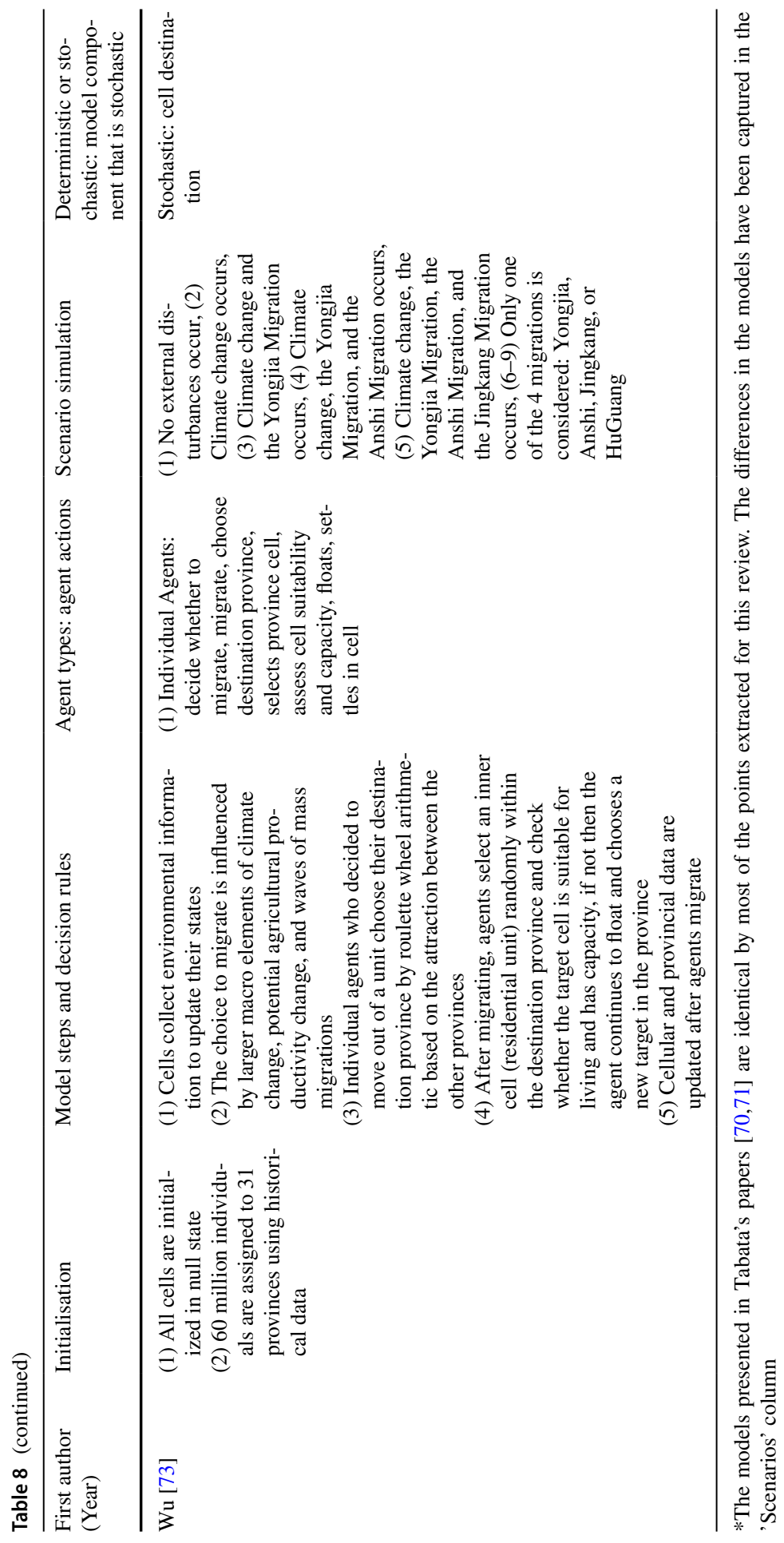


are initialized by constructing the real or abstract spatial setting and populating it with agents set with initial attributes and locations. A commonly repeated theme throughout the models is that an agent primarily decides whether or not to migrate, where to migrate and then attempts to execute migration. In most cases, this decision to migrate is based on a microeconomic utility function, as described in Table 6. Most of the studies $(n=22)$ included a variation of 'scenarios' which usually entailed changing exogenous elements or altering decision rule variables, most commonly this was changes in weather scenarios, but in some cases it was not weather-related, such as differences in employee wages [49] or differences in urban development plans [46].

Table 8 also indicates whether the ABM is deterministic $(n=6)$ or stochastic $(n=22)$, stochastic means that it includes some element of randomness (through specifying probability distributions) in determining agent actions, social network composition, or some element other than the initialization settings. One example of the inclusion of a stochastic process can be found in the Entwisle et al. model where an individual agent's propensity to migrate is compared to a randomly drawn number and if their propensity to migrate is higher than that number then they migrate [50]. Uncertainty in decision-making is pertinent to low-wage labor migration where the migration planning can be unsystematic and the outcomes not guaranteed. Introducing some randomness in decision-making and the migration process reflects some of this uncertainty in outcome. This approach can also model that there is the possibility of divergence from expected decisionmaking behaviors in some cases due either to natural variability in behaviors or uncertainty, which is an acknowledgement that we might not be aware of all the heterogeneous agents' considerations.

5. Model analysis

Finally, we reviewed the main output variables in the analysis of the models, whether or not the authors conducted uncertainty or sensitivity analysis (according to the authors), and if and how the model was validated, again, in the authors' own words (See Table 9). We also extracted the model assumptions and study limitations as described by the authors (See SM-3).

In total, ten studies mentioned some sort of sensitivity testing. Most of these detailed variation testing for a select number of key variables, two studies described using a probability distribution functions of key parameters approach to testing key variables $[47,52]$. When assessing whether or not the models were validated, we relied on the authors' accounts of analysis intended as a form of validation analysis. Twelve studies mentioned some form of model validation (See Table 9). Validation is a challenging task when there is a scarcity of reliable or comparable real-world data. Anderson et al. and Entwisle et al. compared their model outputs with research on a similar topic but using different methods, logistic regression and a system dynamics model, respectively $[47,50]$. Three articles, Fu and Hao, Hassani-Mahmooei and Parris, and Wu et al. all compared the output of their models to available census or population data and Naqvi also compared model outputs to a relevant secondary dataset [52, 55, 73]. Chesney et al. considered validation of the model to be a thorough comparison of whether the model outputs captured the key principles of the Crane's theory on slavery 


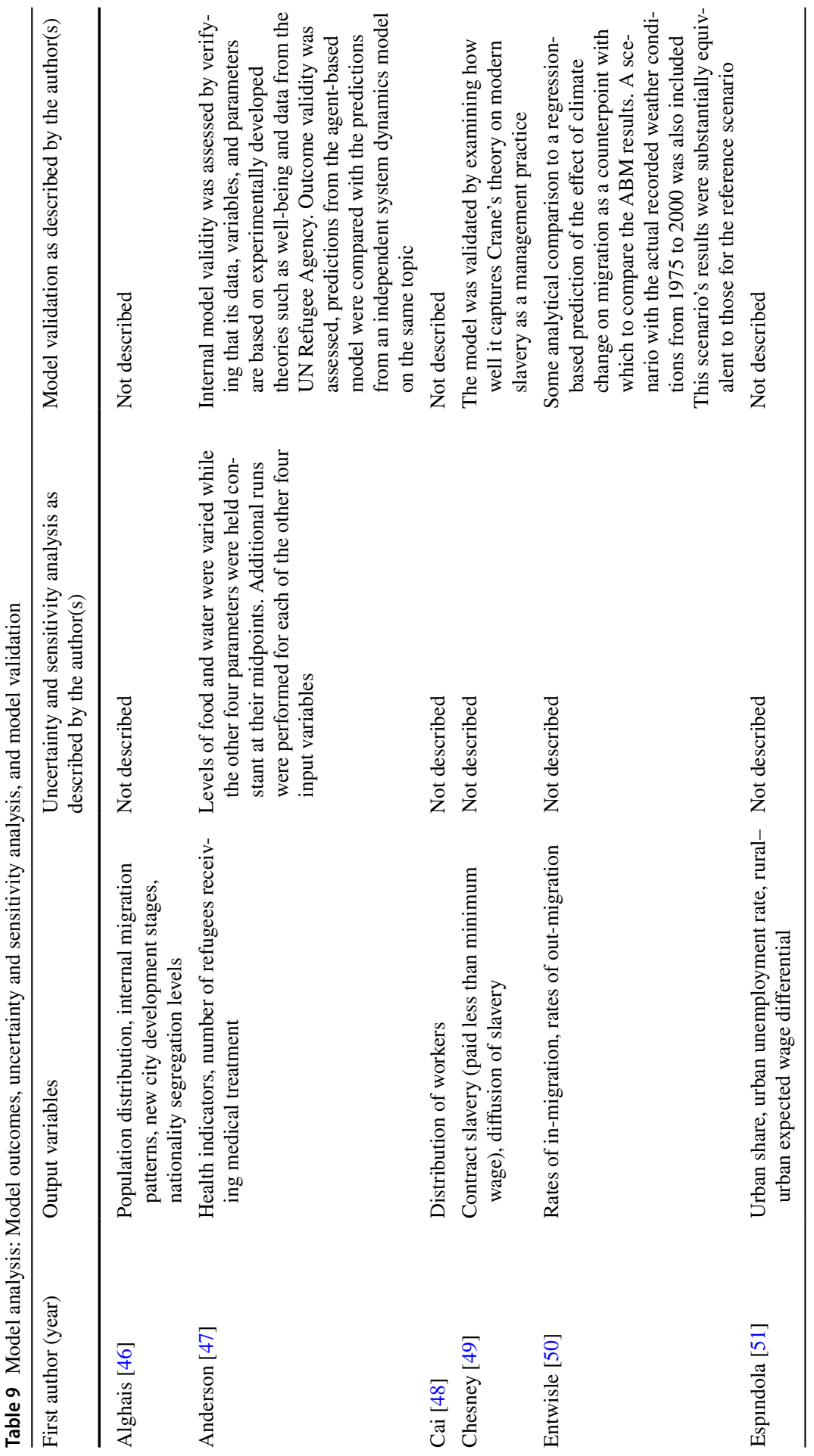




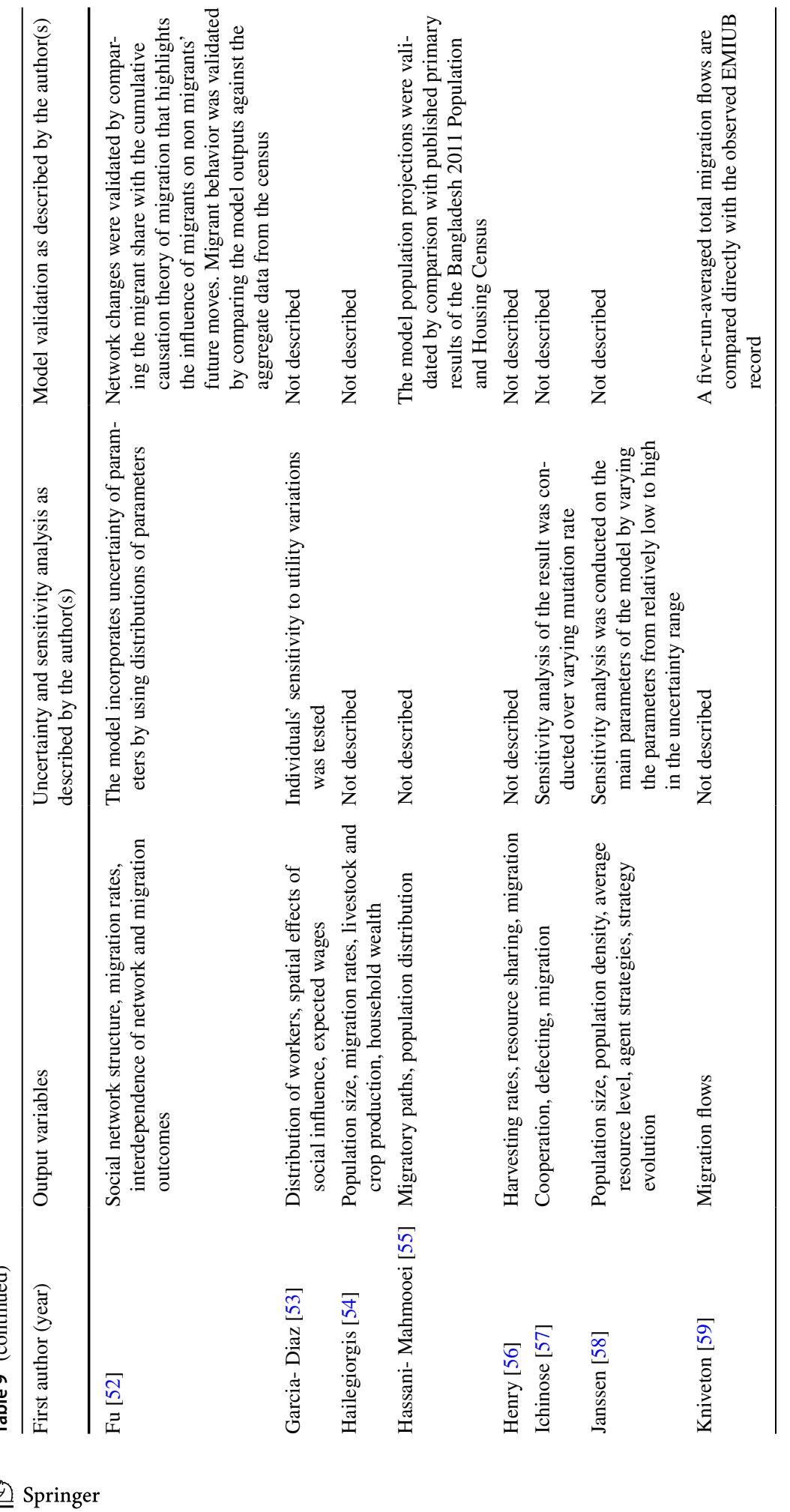




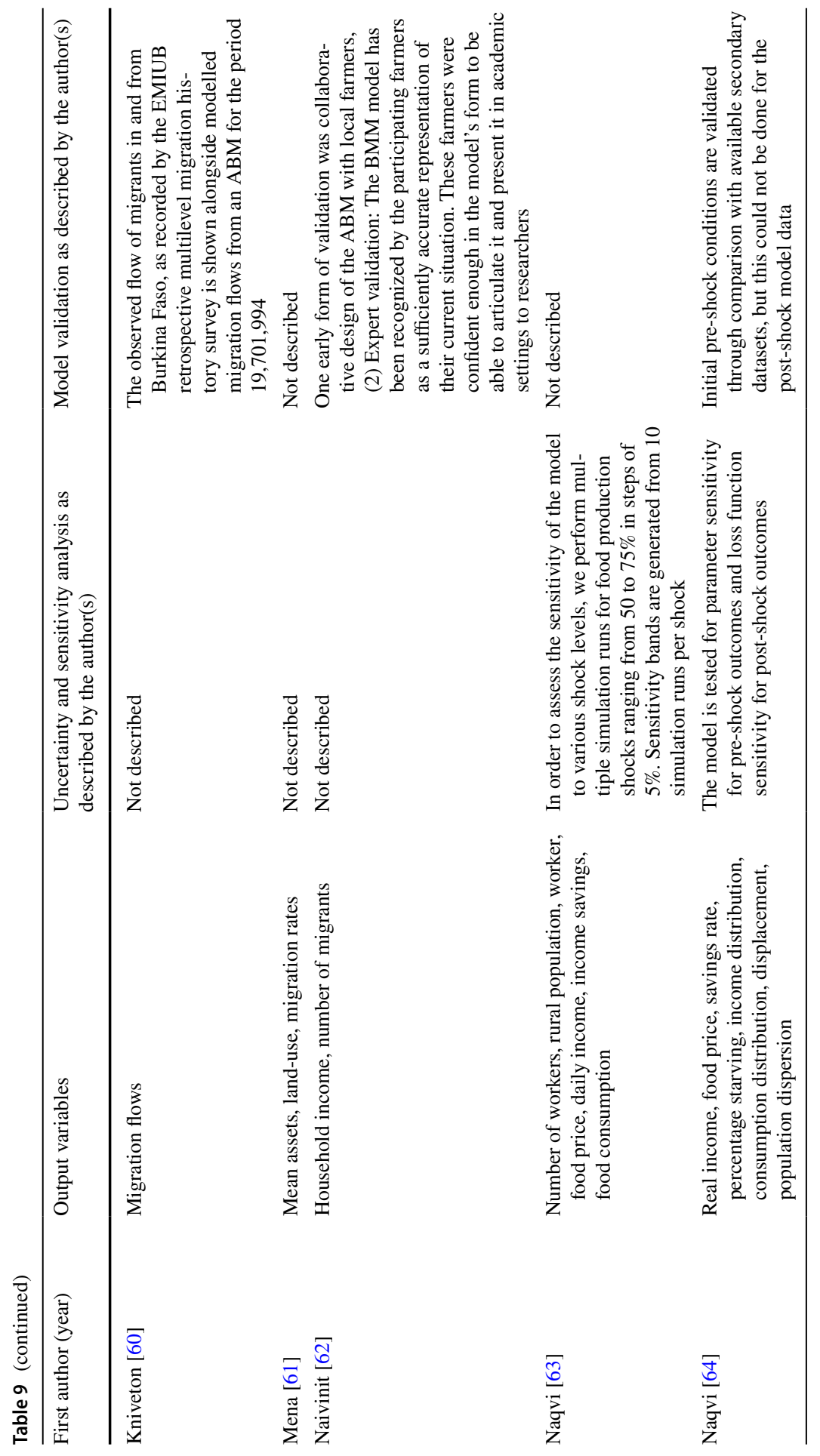




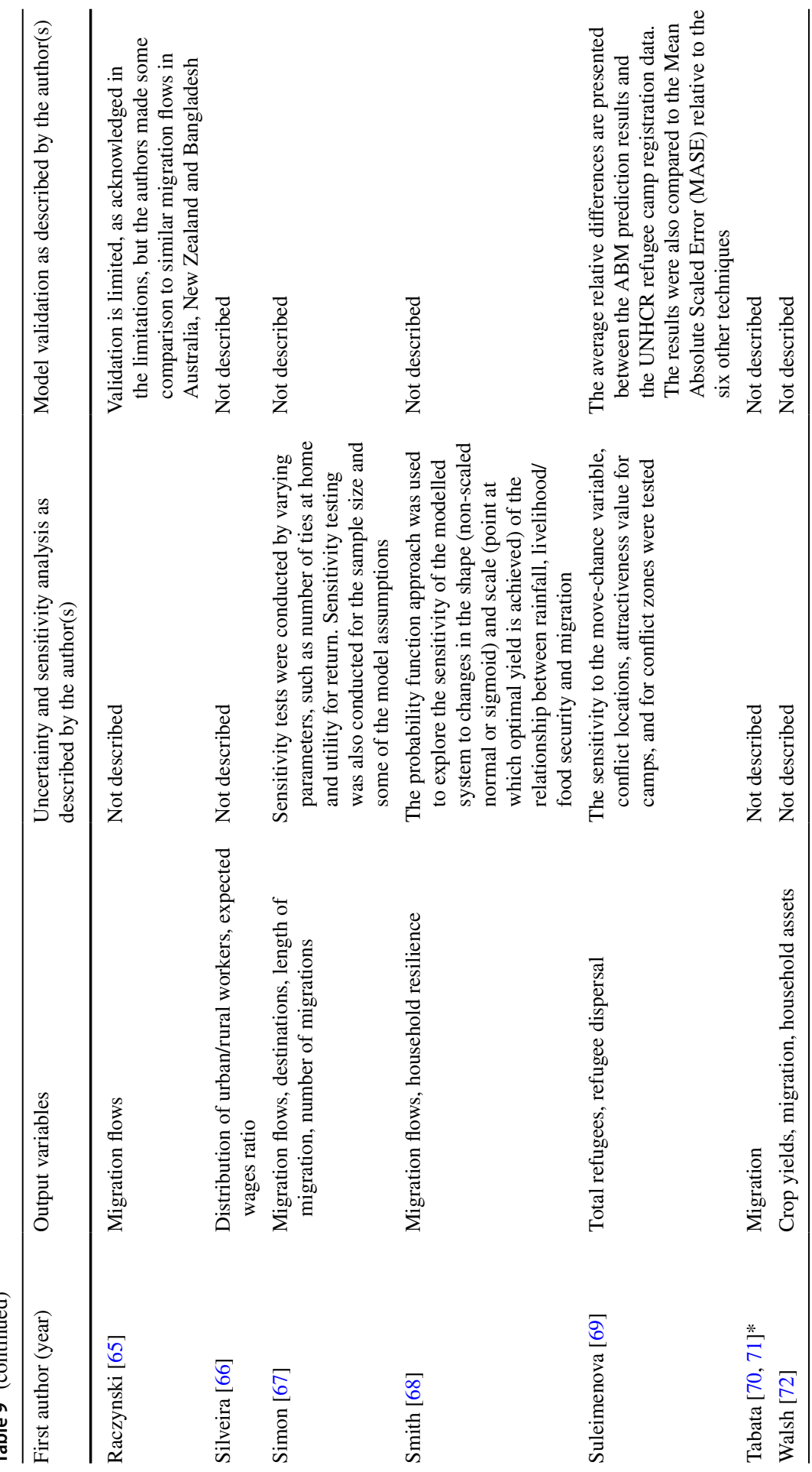




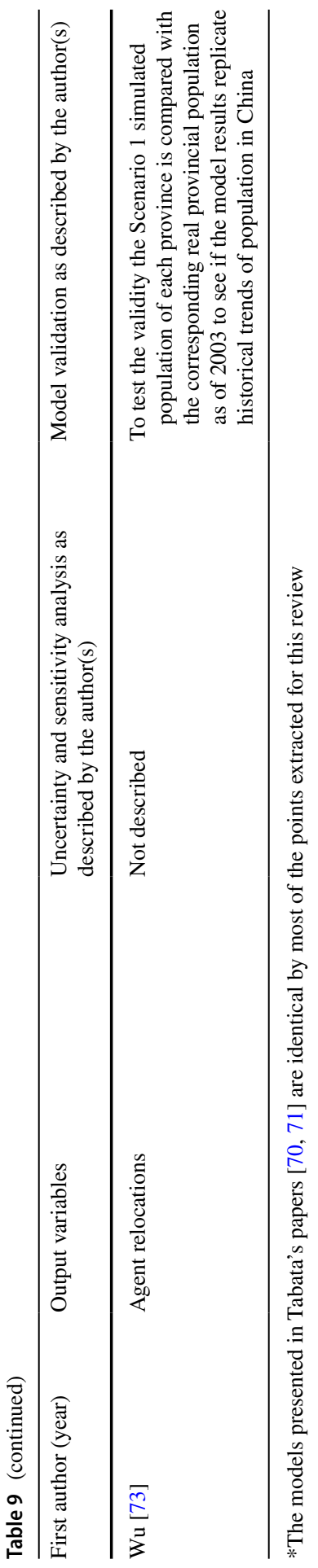




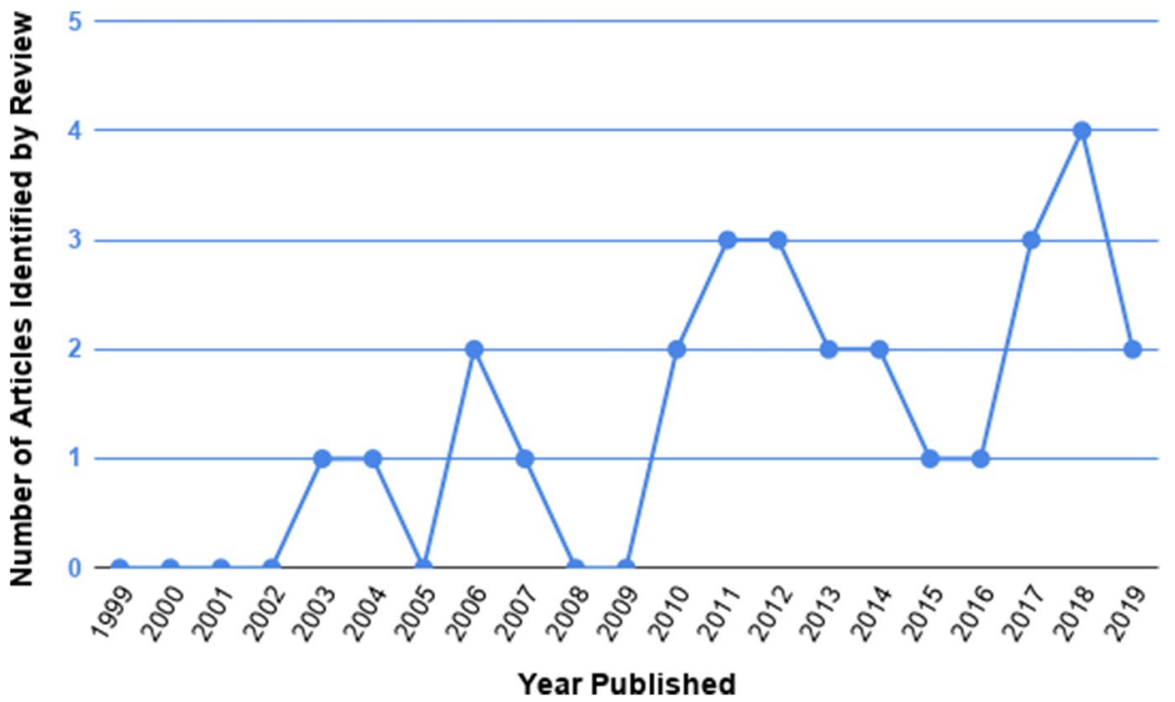

Fig. 4 Included articles by year of publication

as a management practice [49]. In both of the Kniventon et al. studies, the model outputs were compared to the Enquête Migration, Insertion Urbaine et Environnement au Burkina Faso (EMIUB) dataset, which was the same dataset used for calibration of the models $[59,60]$. Naivinit et al. was the only paper that described using stakeholder validation techniques by having key experts review and critique to model [62]. Raczynski struggled to find any available data for validation so instead showed how the model output trends compared to migration flows in three other contexts [65]. Suleimenova et al. used the United Nations High Commissioner for Refugees (UNHCR) refugee camp registration dataset as one comparison point, but then also used the Mean Absolute Scaled Error (MASE) to compare the model outputs to six other model techniques [69]. Again, it was not within the scope of this review to assess the quality of any aspect of the ABM models, so we have not provided quality assessment of the sensitivity analysis, uncertainty analysis or validation methods reported by the authors of these studies.

In relation to sensitivity or uncertainty of outcome variables, a majority of the studies $(n=25)$ looked at least at one migration indicator (migration rates, population distribution, urban vs rural population shares, etc.). Only one study looked at health-related outcomes [47]. Only one study looked at slavery as an outcome [49]. Ten studies looked at some outcome measurement related to financial or resource utility gain (expected earnings, household assets, crop yields, savings, etc.). Only two studies looked at outcomes related to social network structure (such as network dependence and spatial effects of social influence).

6. Correlations between model aim and model development choices

Similar to Klabunde and Willeken's review and Thober and colleagues' review $[2,4]$, this review presents a range of unique models in examining both the model 
aims and model development processes. Due to the lack in standardised reporting of the model development, it is not straightforward to make conclusions about correlations between model aims and model development choices. That said, Table 10 presents a summary of some critical model development choices that we believe relate to the model's purpose. We have grouped the included publications according to their original study aim categorisation (see Table 5) and presented model characteristics that span model calibration, development and process (Tables 6, 7,8). While we again acknowledge that some of the reporting on model development choices was opaque, from the descriptions we were able to extract, we have observed a few correlations between study aims and model development choices.

The majority of ABMs exploring environmental-migration links and humanitarian migration used empirical data, this might reflect the highly context-specific nature of research questions on these topics which require in-depth up-to-date familiarity with the regions, geographies, and events pertinent to the real-world questions. Context specific models on the influence of climate change or humanitarian crisis require empirical embeddedness and would be limited in their claims if they relied only on theory to calibrate models. Likewise, most of the environmental-migration ABMs mapped real geographic environments where these environmental-migration-related phenomena are emerging. While the same importance to context-specific data relates to models investigating modern slavery trends of the social dynamics of migration, there are fewer examples of empirically embedded models, which is more likely to be a reflection of data scarcity in these fields and not a correlation to study aim. The majority of the models exploring socially influenced migration patterns used abstract representations of space which is usually a sufficient simplification of this type of model since the question of interest is social spaces and linkages and is not necessarily concerned with how the abstract representation maps to real geographic space. The models in this review use a range of representations of time-steps. Notable correlations we observed were that the humanitarian migration models use shorter representations (hourly, daily) across all the 4 models which likely reflects the acute timelines of humanitarian crises and the aim to understand large-scale human mobility patterns in short time frames. In comparison, the ABMs aiming to explore migration demographic and regional trends more frequently used annual time-step representations to explore human mobility over longer more regular intervals.

In relation to the inclusion of dynamic interactions, it appears that models that aimed to present environmentally motivated migration all include agent-to-environment interactions, likewise models looking at socially influenced migration all include social networks and agent-to-agent interaction. In comparison, fewer of the models on migration demographic or regional trends include these dynamic interactions. Finally, we thought it was pertinent to note that across all the study aim categorisations there was frequent use of model scenario testing. This is not entirely surprising as this is a key feature and advantage to ABM methods, but it is a particularly useful approach to use on research topics that include high degrees of uncertainty often due to data scarcity or rapid adaptations in individual behav- 


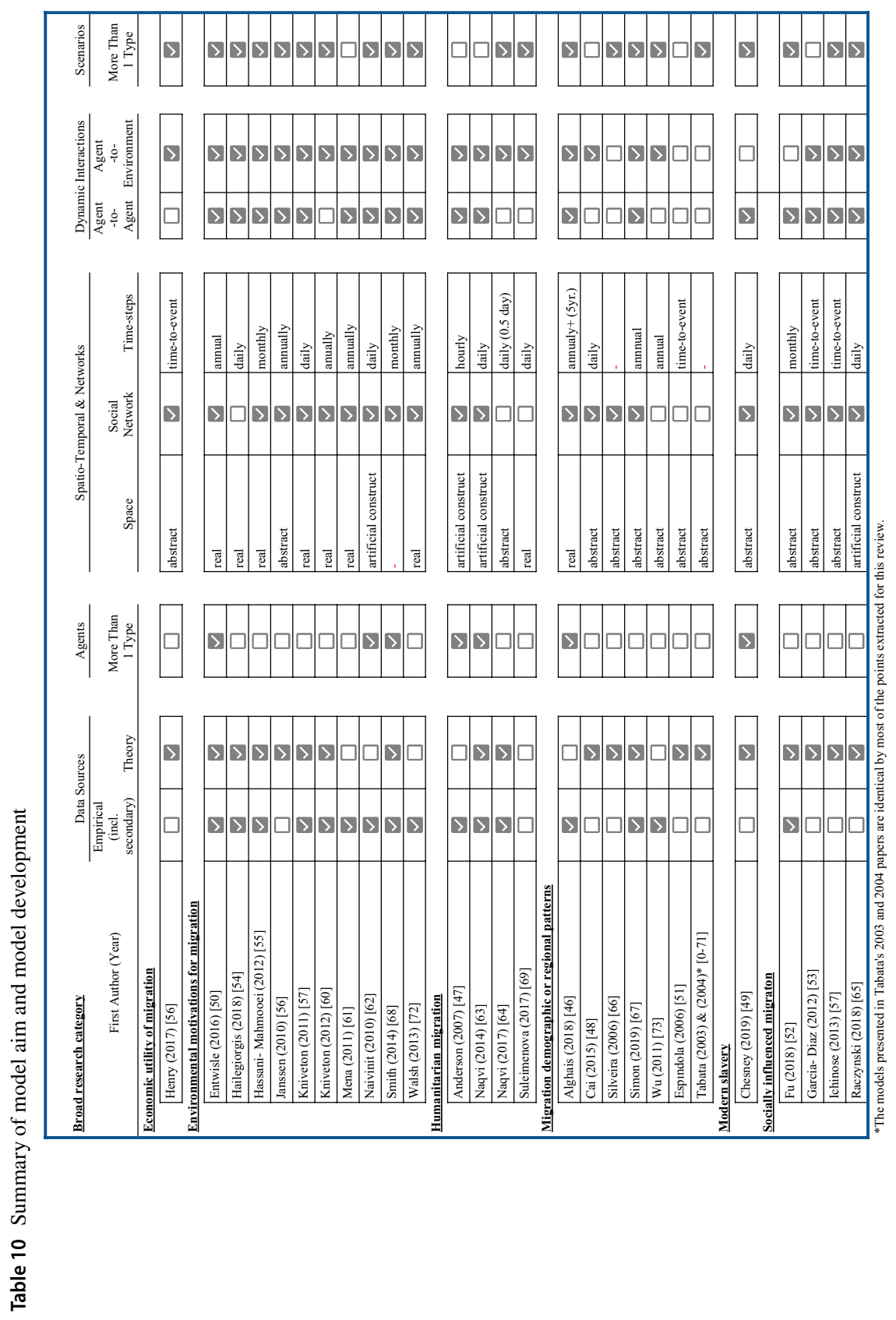




\begin{tabular}{|c|c|c|}
\hline \multicolumn{3}{|l|}{ Table 10 Legend } \\
\hline \multirow[t]{2}{*}{ Data Sources } & $\checkmark$ & $\begin{array}{l}\text { Empirical: Study described the use of at least one primary or secondary empirical datasource for model calibration (this does } \\
\text { not include, published research findings, GIS data, or historical trends in weather data) }\end{array}$ \\
\hline & $\checkmark$ & Theory: Study described the use of theoretical work to inform the model calibration \\
\hline Agents & $\checkmark$ & More Than 1 Type: Model included different agent types (e.g. migrants and employers) \\
\hline \multirow{5}{*}{$\begin{array}{l}\text { Spatio-Temporal } \\
\& \text { Networks }\end{array}$} & $\checkmark$ & Space-Real: Model environment represented a real geographic place (e.g. Northern Thailand), usually mapped using GIS data \\
\hline & $\checkmark$ & $\begin{array}{l}\text { Space-Artificial construct: Model environment represented a structured artificial construct (e.g. refugee camp) that did not } \\
\text { correlate to a real geographic place }\end{array}$ \\
\hline & $\checkmark$ & Space-Abstract: Model environment was an abstract space (e.g. a grid or torus ring) \\
\hline & $\checkmark$ & Social Network: Model included explicit links or ties between agents to form a social network \\
\hline & $\checkmark$ & Time-Steps: How the model represented time-steps in real-time or time-to-event \\
\hline \multirow{2}{*}{$\begin{array}{l}\text { Dynamic } \\
\text { Interactions }\end{array}$} & $\checkmark$ & $\begin{array}{l}\text { Agent-to-Agent: Model included interactions between agents (e.g. exchanges of information or money, influences from social } \\
\text { ties on migration decision, etc.) }\end{array}$ \\
\hline & $\checkmark$ & $\begin{array}{l}\text { Agent-to-Environment: Model included interactions between the agents and the environment or agent information gathering } \\
\text { about the environment (e.g. farming land, assessing destination payoffs to make decisions, etc.) }\end{array}$ \\
\hline Scenarios & $\checkmark$ & More Than 1 Type: Any study that included more than one 'model scenario' that were all tested and compared \\
\hline \multicolumn{3}{|c|}{ Note: '-' = 'information missing' } \\
\hline
\end{tabular}

iour and population trends, which are both true of research on human migration influenced by many dynamic factors, such as changing environments, acute crises, and evolving social networks.

\section{Limitations}

This review does not include structured quality assessments with comparable quality scores. Again, this was partly because the aim of this review was to learn from the methodological approaches not to assess the research findings and the rigor of the findings. As explained in the beginning of the results, models similar to agent-based models but called by different names were excluded for the sake of reproducibility of this review and uniformity in data extraction. We acknowledge that this inclusion criteria may have excluded valuable studies to meet this review's objective and may have introduced a disciplinary bias to this review's inclusion and, therefore, results. To account for this potential loss, we have included these 8 studies in a table in the Supplementary Material (SM-2) for any reader that might be interested to review this specific subset of excluded studies and compare the model development with those included in this review.

This review also did not include some potentially relevant models that have not yet been peer-reviewed but are available on multiple sharing platforms (such as, OpenABM, GitHub, Netlogo libraries). Finally, the large variability in model characteristics makes in-depth synthesis and comparisons of all data extraction points a challenging task. We have attempted and hopefully succeeded at giving a bird's-eye view of the data extraction while not over simplifying the nature of each model by comparing the various elements of the ODD $+\mathrm{D}$ framework too broadly. 


\section{Discussion}

\section{State of the art in ABM in migration and modern slavery research}

The aim and contribution of this review have been to summarize the use of ABM for migration and modern slavery research to present researchers and decision-makers with a comprehensive overview of how ABMs on migration have been developed to date, as well as identifying gaps in ABM development and use in the field. This review highlights that $A B M$ is a method increasingly used in migration research as it has been in other social and health science research topics. To date, ABMs in this field have been used primarily to test theories and less frequently draw on primary empirical input. The main theories modeled in the ABMs identified in this review were utility maximisation theory, social network theory and theory of planned behaviour. Across all the ABMs, about half were neither based on empirical data nor on clearly defined behavioural theories. This is very likely a reflection of the scarcity of empirical data and theoretical frameworks on migration that take a complexity science approach. Many scholars working on social ecological modeling have noted this as an issue across ABMs in many disciplines [1].

The ABMs described in this paper commonly recognised the role of social networks in migration, but few used advanced methods of social network analysis to inform the dynamics of network evolution over time and space. We agree with Klabunde and Willken's assessment that there is still a lot of work to be done in integrating network analysis techniques into ABMs [2]. The papers in this review also noted the complexities in the migration decision and processes. However, with the exception of Suleimenova (2017) [69], most models assumed that migrants were "rational agents", for example that migrants knew the target labor markets at destination, that the choice of destination was open and informed, and that deception was not a possibility. Many of these models did not capture all of the core elements of decision-making, notably missing are the evaluation of migration options or complexity in the decision process that Klabunde and Willeken's emphasized in their review [2]. For instance, going back to our earlier example of research on the nexus of low-wage labour migration and modern slavery, none of the labour migration models in this review included the risks of labor exploitation and how this might influence migration decision-making or expected utility calculations. In that respect, these models do not capture the experiences of most low-wage labor migrants globally. The challenge is that there is a knowledge gap on how the risks of labor exploitation or other forms of modern slavery might influence individual behaviors. There are many areas we still do not understand the underpinning mechanisms of entry into modern slavery versus decent work and thus the models trying to represent lowwage labor migration are not transferable to real-world interventions. The choice of stochastic models may compensate to some extent for these knowledge gaps or further unknown heterogeneity in migration behaviours and outcomes. However, it is also important to note that some uncertainty cannot be reduced by acquiring knowledge, such as variability in agents' behaviors. Thus the stochastic variability will always be essential to account for some of these irreducible uncertainties. However, 
the consideration of decision-model options and choice is a critical step in ensuring models capture the complexity of real-world processes. Klabunde and Willeken's review provides in-depth recommendations on the way forward to capturing migration decision-models in ABMs. Scholars exploring the intersection of cognition and multi-agent systems have emphasised opportunities to integrate cognitive modelling into social studies addressing complex behaviors that originate in individual perceptions [80].

Additionally, the models' development varied considerably in terms of attributes and interactions of agents, time, space, and environment. The most common model processes involve scenarios where an agent decides whether or not to migrate, where to migrate to, and then attempts to execute the act of migration. Changes in scenarios were introduced in ABMs by changing exogenous elements or altering decision rules, but the majority of these were climate scenarios and these models would benefit from a broader exploration of influences on individual migration behaviors as Bell and colleagues also recommend in their recent review [3].

Finally, many of the ABMs did not undergo validation or sensitivity analysis, which are critical steps to ensuring rigor in ABM methods and confidence in their use to inform policy makers. Model validation is an area of work critical to all ABM practice and methods of empirical validation are still in their infancy as are standardised best practice of model analysis [1,81]. While there are serious challenges to validating ABMs modeling in data-scarce topics, such as this, there are, nonetheless, validation methods that are feasible and acceptable given these challenges [27]. The major challenge concerns validating the ABM against qualitative data in part because much of these data have not been collected with developing ABMs in mind and it can be difficult to calibrate or validate the decision-rules from these data sources. There is an opportunity for methodological development in translating qualitative analysis for use in ABMs. Even small samples of key expert interviews can be critical in informing the model development and justifying the model design [76].

\section{Future considerations for migration ABMs}

Migration complexity research will benefit from studies that collect primary data on migration and migration outcomes, such as modern slavery, with ABMs in mind from the research design conception stage. Going back to our migration and modern slavery example, researchers can collect information specifically about the agents (migrants, brokers, intermediaries, employers), agent-attributes (migration experiences, migration knowledge, resources to migrate), agent-agent interactions (who influences migrants' attitudes toward migration, how migrants meet brokers, how migrants find or change employers at destination), agent-environment interactions (how migrants engage with or avoid institutions managing legal labor migration pathways), and most importantly, how individuals and groups make decisions, learn and adapt (the emergence of preferred migration pathways over time). A further benefit of ABM methods and future consideration in study design is that multiple data sources can be incorporated from several disciplines. This review presents 
examples of how ABMs can use multiple data sources, but one method that has been under-utilized in this group of studies is participatory methods with key stakeholder groups and the target population of interest, which have offered critical insights to other nascent fields of computational social science research. For example, the stakeholder approach allows modelers with perhaps in-depth theoretical knowledge on the topic to collaborate with domain experts and triangulate these sources of established theory and current expert knowledge [76]. There was one example in this review that used this stakeholder approach in a series of workshops with Thai farmers to inform the individual behavioural rules of the model to reflect the Thai context. An interdisciplinary approach is critical to be able to integrate theoretical and empirical knowledge of a social phenomenon into a mathematical model [20]. In fact, we concur with the other reviews, future ABM work in this area would benefit from interdisciplinary approaches to every stage of model development to address research questions that cut across social and ecological studies, as well as disciplines such as economics, psychology, geography, and complexity science [2, 4]. Diverse disciplinary and theoretical perspectives will strengthen the real-world likeness of these highly complex, dynamic, and socially embedded models and may present new uses for existing data for model calibration.

Lastly, despite widely accepted challenges and shortcomings to standardising model analysis procedures and model validation, all future ABM studies must critically consider their approaches to these steps, learn from ABM practices across all disciplines, and document the justifications for their analysis and validation choices as well as their process to enable a wider and more synchronised debate on future best practice.

\section{The need for transparent and clear reporting on model development}

The model descriptions in the articles included in this review were overwhelmingly opaque with various critical details omitted. This review is not the first to critique the lack of a 'best practice' in reporting model development $[1,4]$ and as one reviewer noted there are severe limitations to systematic reviews that aim to synthesise findings across a body of studies whose methods cannot be easily compared, not to mention that there is still work to be done to develop standard methods for systematically reviewing ABMs [34].

In this review, there was generally insufficient detail or models were reported without proper sign-posting or terminology that could be understood by most readers. Since few of the studies used recommended development frameworks, such as $\mathrm{ODD}+\mathrm{D}$, there was not a structure or fluidity to the way the model choices were reported, which makes it difficult to interpret findings and learn from the methods at a critical stage in methodological development for the field of migration and modern slavery research. This critique is true for ABM research on other topics as well. This review suggests that more frequent use of the ODD $+\mathrm{D}$ framework will strengthen future $\mathrm{ABM}$ research.

In addition, the methods of sensitivity analysis and model validation were described in limited detail or not covered by the authors, which leads us to assume 
that the analysis was either not done or not completed. The findings of the models arguably are then open to substantial criticism regarding their validity and applied usefulness. This weakness is a critical gap in the evidence-base since the topics of migration and modern slavery are priorities on many global agendas, including the beforementioned UN Sustainable Development Goals [6].

\section{Using ABM to address pressing and complex questions in the fields of migration and modern slavery research}

Migration is currently a focus of substantial international attention, particularly global economic migration and modern forms of slavery. Policy-makers are seeking a strong evidence-base that can be used to address the myriad aspects of global mobility. Current pressing concerns include migration patterns and trajectories, individual or group vulnerability, and migrant protection and safety. To date, robust evidence to inform policy-making has proven to be weak because individual and population mobility cannot be explained by the exclusive use of methods that assume linear average effects of exposures on single outcomes affecting homogenous populations. The patterns of migration and characteristics of migrants are emergent consequences of many interacting and non-linear, unpredictable phenomena, and this complexity requires an agent paradigm approach [14].

For example, many of the current interventions and policy goals for safer migration are currently relying on extremely simplistic cause and effect assumptions about the exposures that might lead to modern slavery. If we are going to achieve SDG 8.7- "eradicate forced labour, end modern slavery and human trafficking and secure the prohibition and elimination of the worst forms of child labour, including recruitment and use of child soldiers"-we must engage with the complexity and dynamics of low-wage labor migration and population-level emergent outcomes (such as widespread labor exploitation) in our research methodologies. For example, the influence of social networks in group decision-making must be explored in greater depth and this will give rise to a greater understanding of how these interactions might hinder the effectiveness of migration interventions that operate on the individual level [28].

To study migration, one has to go beyond simple binaries, such as: domestic or international; documented or undocumented; recruiter facilitated or social network facilitated; forced or voluntary; for asylum or for work, etc. Because migration is a mechanistic and dynamic process, analyses must be able to address this complexity.

Agent-based modeling offers a promising method to improve our understanding of and programmatic responses to these real-world problems. This review offers an overview on how ABM has been used in the fields of migration and modern slavery research and the remaining gaps and potential future uses to advance the application of these methods to inform more effective responses to high-risk migration and modern slavery.

Acknowledgements This systematic review and preparation of this article have been completed as part of Alys McAlpine's Doctoral work, which has received generous support from the Economic and Social 
Research Council (ESRC) as part of The UCL, Bloomsbury and East London (UBEL) Doctoral Training Partnership (DTP) scholarships scheme and The Freedom Fund.

Author contributions Alys McAlpine had the idea for this review as part of her Doctoral degree. All authors contributed to the review conception and design. Alys McAlpine and Ligia Kiss conducted the literature search and review screening. Alys McAlpine conducted the data extraction and data analysis. Zaid Chalabi and Ligia Kiss conducted the quality assessment of the data extraction. Alys McAlpine wrote the first draft of the article. All authors conducted critically revised the work through multiple thorough reviews and group discussions.

Funding This review was completed as part of Alys McAlpine's Doctoral degree which is receiving generous funding from the Economic and Social Research Council and The Freedom Fnud in the form of a Doctoral Training Partnership award.

Data availability The full data extraction table for this systematic review can be found in the Supplementary Material. All data extraction is from the cited articles' text or the articles' supplementary material text.

\section{Compliance with ethical standard}

Conflicts of interest This review and its authors do not have any conflicts of interest pertaining to this work.

Open Access This article is licensed under a Creative Commons Attribution 4.0 International License, which permits use, sharing, adaptation, distribution and reproduction in any medium or format, as long as you give appropriate credit to the original author(s) and the source, provide a link to the Creative Commons licence, and indicate if changes were made. The images or other third party material in this article are included in the article's Creative Commons licence, unless indicated otherwise in a credit line to the material. If material is not included in the article's Creative Commons licence and your intended use is not permitted by statutory regulation or exceeds the permitted use, you will need to obtain permission directly from the copyright holder. To view a copy of this licence, visit http://creativecommons.org/licen ses/by/4.0/.

\section{References}

1. Schulze, J., Müller, B., Groeneveld, J., \& Grimm, V. (2017). Agent-Based Modelling of SocialEcological Systems: Achievements, Challenges, and a Way Forward. Journal of Artificial Societies and Social Simulation, 20(2), 8.

2. Klabunde, A., \& Willekens, F. (2016). Decision-Making in Agent-Based Models of Migration: State of the Art and Challenges. European Journal of Population, 32(1), 73-97. https://doi.org/10.1007/ s10680-015-9362-0.

3. Bell, A., Hernandez, C. C., \& Oppenheimer, M. (2019). Migration, Intensification, and Diversification as Adaptive Strategies. Socio-Environmental Systems Modelling, 1, 16102. https://doi. org/10.18174/sesmo.2019a16102.

4. Thober, J., Schwarz, N., \& Hermans, K. (2018). Agent-based modeling of environment-migration linkages: A review. Ecology and Society. https://doi.org/10.5751/ES-10200-230241.

5. Müller, B., Bohn, F., Dreßler, G., Groeneveld, J., Klassert, C., Martin, R., et al. (2013). Describing human decisions in agent-based models - ODD + D, an extension of the ODD protocol. Environmental Modelling \& Software, 48, 37-48. https://doi.org/10.1016/j.envsoft.2013.06.003.

6. United Nations. (2020). The Sustainable Development Goals Indicators. Resource Document. United Nations. https://www.unstats.un.org/sdgs/indicators/indicatorslist/. Accessed 6 Mar 2020.

7. LeBaron, G., Kyritsis, P., Thibos, C., \& Howard, N. (2019). Confronting root causes: forced labour in global supply chains. Resource Document. OpenDemocracy. https://www.opendemocracy.net/en/ beyond-trafficking-and-slavery/confronting-root-causes/. Accessed 6 Mar 2020. 
8. Zimmerman, C., \& Kiss, L. (2017). Human trafficking and exploitation: A global health concern. PLOS Medicine, 14(11), e1002437. https://doi.org/10.1371/journal.pmed.1002437.

9. Zimmerman, C., McAlpine, A., \& Kiss, L. (2015). Safer labour migration and community-based prevention of exploitation: The state of the evidence for programming. Resource Document. The Freedom Fund and The London School of Hygiene and Tropical Medicine. https://freedomfund.org/ our-reports/safer-labour-migration-and-community-based-prevention-of-exploitation-the-state-ofthe-evidence-for-programming/. Accessed 6 Mar 2020.

10. International Labour Organisation. (2017). Global Estimates of Modern Slavery: Forced labour and forced marriage. Geneva: ILO.

11. International Labour Office. (2018). ILO Global Estimates on International Migrant Workers: Results and Methodology. Resource Document. International Labour Organization. https://www.ilo. org/global/publications/books/WCMS_652001/lang--en/index.htm. Accessed 6 Mar 2020.

12. Keyes, K., \& Galea, S. (2015). What matters most: quantifying an epidemiology of consequence. Annals of epidemiology, 25(5), 305-311. https://doi.org/10.1016/j.annepidem.2015.01.016.

13. Hemmings, S., Jakobowitz, S., Abas, M., Bick, D., Howard, L. M., Stanley, N., et al. (2016). Responding to the health needs of survivors of human trafficking: a systematic review. BMC Health Services Research, 16(1), 320. https://doi.org/10.1186/s12913-016-1538-8.

14. Marshall, B. D. L., \& Galea, S. (2015). Formalizing the role of agent-based modeling in causal inference and epidemiology. American Journal of Epidemiology, 181(2), 92-99. https://doi. org/10.1093/aje/kwu274.

15. Borghi, J., \& Chalabi, Z. (2017). Square peg in a round hole: re-thinking our approach to evaluating health system strengthening in low-income and middle-income countries. BMJ Global Health. https ://doi.org/10.1136/bmjgh-2017-000406.

16. El-Sayed, A. M., Scarborough, P., Seemann, L., \& Galea, S. (2012). Social network analysis and agent-based modeling in social epidemiology. Epidemiologic Perspectives \& Innovations, 9(1), 1. https://doi.org/10.1186/1742-5573-9-1.

17. Epstein, J. (2006). Generative Social Science: Studies in Agent-Based Computational Models. Princeton, NJ: Princeton University Press.

18. Bonabeau, E. (2002). Agent-based modeling: Methods and techniques for simulating human systems. Proceedings of the National Academy of Sciences, 99(suppl 3), 7280-7287. https://doi. org/10.1073/pnas.082080899.

19. Cioffi-Revilla, C. (2014). Introduction to Computational Social Science: Principles and Applications. London: Springer-Verlag. https://doi.org/10.1007/978-1-4471-5661-1.

20. Wolfram, S. (1988). Complex Systems Theory. In S. Wolfram (Ed.), Cellular Automata and Complexity: Collected Papers (pp. 491-497). Boulder, CO: Westview Press.

21. Tracy, M., Cerdá, M., \& Keyes, K. M. (2018). Agent-Based Modeling in Public Health: Current Applications and Future Directions. Annual Review of Public Health, 39(1), 77-94. https://doi. org/10.1146/annurev-publhealth-040617-014317.

22. Chalabi, Z., \& Lorenc, T. (2013). Using agent-based models to inform evaluation of complex interventions: Examples from the built environment. Preventive Medicine, 57(5), 434-435. https://doi. org/10.1016/j.ypmed.2013.07.013.

23. Epstein, J. M., \& Axtell, R. (1996). Growing Artificial Societies: Social Science from the Bottom Up. Washington, D.C.: Brookings Institution Press.

24. Gomersall, T. (2018). Complex adaptive systems: a new approach for understanding health practices. Health Psychology Review, 12(4), 405-418. https://doi.org/10.1080/17437199.2018.1488603.

25. Conte, R., \& Paolucci, M. (2014). On agent-based modeling and computational social science. Frontiers in Psychology. https://doi.org/10.3389/fpsyg.2014.00668.

26. Wilensky, U., \& Rand, W. (2015). An introduction to agent-based modeling: modeling natural, social, and engineered complex systems with NetLogo. Cambridge, Massachusetts: The MIT Press.

27. Badham, J., Chattoe-Brown, E., Gilbert, N., Chalabi, Z., Kee, F., \& Hunter, R. F. (2018). Developing agent-based models of complex health behaviour. Health \& Place, 54, 170-177. https://doi. org/10.1016/j.healthplace.2018.08.022.

28. Haug, D. S. (2008). Migration Networks and Migration Decision-Making. Journal of Ethnic and Migration Studies, 34(4), 585-605. https://doi.org/10.1080/13691830801961605.

29. Zimmerman, C., Kiss, L., \& Hossain, M. (2011). Migration and Health: A Framework for 21st Century Policy-Making. PLOS Medicine, 8(5), e1001034. https://doi.org/10.1371/journal.pmed.10010 34. 
30. Kaplan, G. A., Roux, A. V. D., Simon, C. P., \& Galea, S. (2017). Growing Inequality: Bridging Complex Systems, Population Health, and Health Disparities. Washington, D.C.: Westphalia Press.

31. El-Sayed, A. M., \& Galea, S. (2017). Systems Science and Population Health. Oxford: Oxford University Press.

32. Groff, E. R., Johnson, S. D., \& Thornton, A. (2019). State of the Art in Agent-Based Modeling of Urban Crime: An Overview. Journal of Quantitative Criminology, 35(1), 155-193. https://doi. org/10.1007/s10940-018-9376-y.

33. Nianogo, R. A., \& Arah, O. A. (2015). Agent-Based Modeling of Noncommunicable Diseases: A Systematic Review. American Journal of Public Health, 105(3), e20-e31. https://doi.org/10.2105/ AJPH.2014.302426.

34. Morshed, A. B., Kasman, M., Heuberger, B., Hammond, R. A., \& Hovmand, P. S. (2019). A systematic review of system dynamics and agent-based obesity models: Evaluating obesity as part of the global syndemic. Obesity Reviews, 20(S2), 161-178. https://doi.org/10.1111/obr.12877.

35. Cassidy, R., Singh, N. S., Schiratti, P.-R., Semwanga, A., Binyaruka, P., Sachingongu, N., et al. (2019). Mathematical modelling for health systems research: a systematic review of system dynamics and agent-based models. BMC Health Services Research, 19(1), 845. https://doi.org/10.1186/ s12913-019-4627-7.

36. Moher, D., Liberati, A., Tetzlaff, J., Altman, D. G., \& Group, T. P. (2009). Preferred Reporting Items for Systematic Reviews and Meta-Analyses: The PRISMA Statement. PLOS Medicine, 6(7), e1000097. https://doi.org/10.1371/journal.pmed.1000097.

37. Rayyan QCRI. (2020). Resource Document. https://rayyan.qcri.org/. Accesed 21 Feb 2020.

38. Smith, N. R., Trauer, J. M., Gambhir, M., Richards, J. S., Maude, R. J., Keith, J. M., et al. (2018). Agent-based models of malaria transmission: a systematic review. Malaria Journal, 17(1), 299. https://doi.org/10.1186/s12936-018-2442-y.

39. de Amorim Silva, R., \& Braga, R. T. V. (2020). Simulating Systems-of-Systems With Agent-Based Modeling: A Systematic Literature Review. IEEE Systems Journal. https://doi.org/10.1109/JSYST .2020 .2980896 .

40. Barbati, M., Bruno, G., \& Genovese, A. (2012). Applications of agent-based models for optimization problems: A literature review. Expert Systems with Applications, 39(5), 6020-6028. https://doi. org/10.1016/j.eswa.2011.12.015.

41. Chen, L. (2012). Agent-based modeling in urban and architectural research: A brief literature review. Frontiers of Architectural Research, 1(2), 166-177. https://doi.org/10.1016/j.foar.2012.03.003.

42. Grimm, V., Berger, U., Bastiansen, F., Eliassen, S., Ginot, V., Giske, J., et al. (2006). A standard protocol for describing individual-based and agent-based models. Ecological Modelling, 198(1-2), 115-126. https://doi.org/10.1016/j.ecolmodel.2006.04.023.

43. Grimm, V., Berger, U., DeAngelis, D. L., Polhill, J. G., Giske, J., \& Railsback, S. F. (2010). The ODD protocol: A review and first update. Ecological Modelling, 221(23), 2760-2768. https://doi. org/10.1016/j.ecolmodel.2010.08.019.

44. Soilemezi, D., \& Linceviciute, S. (2018). Synthesizing Qualitative Research: Reflections and Lessons Learnt by Two New Reviewers. International Journal of Qualitative Methods, 17(1), 1609406918768014. https://doi.org/10.1177/1609406918768014.

45. CASP UK. (2020). CASP Checklists. Resource Document. CASP - Critical Appraisal Skills Programme. https://www.casp-uk.net/casp-tools-checklists/. Accessed 6 Mar 2020.

46. Alghais, N., Pullar, D., \& Charles-Edwards, E. (2018). Accounting for peoples' preferences in establishing new cities: A spatial model of population migration in Kuwait. PLoS ONE, 13(12), e0209065. https://doi.org/10.1371/journal.pone.0209065.

47. Anderson, J., Chaturvedi, A., \& Cibulskis, M. (2007). Simulation tools for developing policies for complex systems: Modeling the health and safety of refugee communities. Health Care Management Science, 10(4), 331-339. https://doi.org/10.1007/s10729-007-9030-y.

48. Cai, N., Ma, H.-Y., \& Khan, M. J. (2015). Agent-based model for rural-urban migration: a dynamic consideration. Physica A. Statistical Mechanics and its Applications, 436, 806-813. https://doi. org/10.1016/j.physa.2015.05.015.

49. Chesney, T., Evans, K., Gold, S., \& Trautrims, A. (2019). Understanding labour exploitation in the Spanish agricultural sector using an agent based approach. Journal of Cleaner Production, 214, 696-704.

50. Entwisle, B., Williams, N. E., Verdery, A. M., Rindfuss, R. R., Walsh, S. J., Malanson, G. P., et al. (2016). Climate shocks and migration: an agent-based modeling approach. Population and Environment, 38(1), 47-71. https://doi.org/10.1007/s11111-016-0254-y. 
51. Espindola, A., Silveira, J., Penna, T., Espindola, A. L., Silveira, J. J., \& Penna, T. J. P. (2006). A Harris-Todaro agent-based model to rural-urban migration. Brazilian Journal of Physics, 36(3), 603-609.

52. Fu, Z., \& Hao, L. (2018). Agent-based modeling of China's rural-urban migration and social network structure. Physica A: Statistical Mechanics and its Applications, 490, 1061-1075. https://doi. org/10.1016/j.physa.2017.08.145.

53. Garcia-Diaz, C., \& Moreno-Monroy, A. (2012). Social influence, agent heterogeneity and the emergence of the urban informal sector. Physica A-Statistical Mechanics and its Applications, 391(4), 1563-1574.

54. Hailegiorgis, A., Crooks, A., \& Cioffi-Revilla, C. (2018). An agent-based model of rural households' adaptation to climate change. Journal of Artificial Societies and Social Simulation, 21(4), 4.

55. Hassani-Mahmooei, B., \& Parris, B. W. (2012). Climate change and internal migration patterns in Bangladesh: An agent-based model. Environment and Development Economics, 17(6), 763-780. https://doi.org/10.1017/S1355770X12000290.

56. Henry, A. D., Christensen, A. E., Hofmann, R., Steimanis, I., \& Vollan, B. (2017). Influence of sea level rise on discounting, resource use and migration in small-island communities: An agent-based modelling approach. Environmental Conservation, 44(4), 381-388. https://doi.org/10.1017/S0376 892917000339.

57. Ichinose, G., Saito, M., Sayama, H., \& Wilson, D. S. (2013). Adaptive long-range migration promotes cooperation under tempting conditions. Scientific Reports. https://doi.org/10.1038/srep02509.

58. Janssen, M. A. (2010). Population aggregation in ancient arid environments. Ecology and Society, 15(2), 15. https://doi.org/10.5751/ES-03376-150219.

59. Kniventon, D., Smith, C., \& Wood, S. (2011). Agent-based model simulations of future changes in migration flows for Burkina Faso. Global Environmental Change-Human and Policy Dimensions, 21, S34-S40.

60. Kniventon, D., Smith, C., \& Black, R. (2012). Emerging migration flows in a changing climate in dryland Africa. Nature Climate Change, 2(6), 444-447.

61. Mena, C. F., Walsh, S. J., Frizzelle, B. G., Xiaozheng, Y., \& Malanson, G. P. (2011). Land use change on household farms in the Ecuadorian Amazon: Design and implementation of an agentbased model. Applied Geography, 31(1), 210-222. https://doi.org/10.1016/j.apgeog.2010.04.005.

62. Naivinit, W., Le Page, C., Trébuil, G., \& Gajaseni, N. (2010). Participatory agent-based modeling and simulation of rice production and labor migrations in Northeast Thailand. Environmental Modelling and Software, 25(11), 1345-1358. https://doi.org/10.1016/j.envsoft.2010.01.012.

63. Naqvi, A. A., \& Rehm, M. (2014). A multi-agent model of a low income economy: simulating the distributional effects of natural disasters. Journal of Economic Interaction and Coordination, 9(2), 275-309. https://doi.org/10.1007/s11403-014-0137-1.

64. Naqvi, A. (2017). Deep Impact: Geo-Simulations as a Policy Toolkit for Natural Disasters. World Development, 99, 395-418. https://doi.org/10.1016/j.worlddev.2017.05.015.

65. Raczynski, S. (2018). Influence of the gregarious instinct and individuals' behavior patterns on macro migrations: Simulation experiments. Journal of Human Behavior in the Social Environment, 28(2), 204-220. https://doi.org/10.1080/10911359.2017.1388758.

66. Silveira, J. J., Espíndola, A. L., \& Penna, T. J. P. (2006). Agent-based model to rural-urban migration analysis. Physica A: Statistical Mechanics and its Applications, 364, 445-456. https://doi. org/10.1016/j.physa.2005.08.055.

67. Simon, M. (2019). Path Dependency and Adaptation: The Effects of Policy on Migration Systems. Journal of Artificial Societies and Social Simulation, 22(2), 2.

68. Smith, C. D. (2014). Modelling migration futures: Development and testing of the Rainfalls Agent-Based Migration Model - Tanzania. Climate and Development, 6(1), 77-91. https://doi. org/10.1080/17565529.2013.872593.

69. Suleimenova, D., Bell, D., \& Groen, D. (2017). A generalized simulation development approach for predicting refugee destinations. Scientific Reports. https://doi.org/10.1038/s41598-017-13828-9.

70. Tabata, M., \& Eshima, N. (2003). A self-referential agent-based model that consists of a large number of agents moving stochastically in a discrete bounded domain. Applied Mathematics and Computation, 143(2-3), 443-483. https://doi.org/10.1016/S0096-3003(02)00375-2.

71. Tabata, M., \& Eshima, N. (2004). The behavior of stochastic agent-based models when the number of agents and the time variable tend to infinity. Applied Mathematics and Computation, 152(1), 47-70. https://doi.org/10.1016/S0096-3003(03)00544-7. 
72. Walsh, S. J., Malanson, G. P., Entwisle, B., Rindfuss, R. R., Mucha, P. J., Heumann, B. W., et al. (2013). Design of an agent-based model to examine population-environment interactions in Nang Rong District, Thailand. Applied Geography, 39, 183-198. https://doi.org/10.1016/j.apgeo g.2012.12.010.

73. Wu, J., Mohamed, R., \& Wang, Z. (2011). Agent-based simulation of the spatial evolution of the historical population in China. Journal of Historical Geography, 37(1), 12-21. https://doi. org/10.1016/j.jhg.2010.03.006.

74. Heppenstall, A. J. (Ed.). (2012). Agent-based models of geographical systems. Dordrecht: Springer.

75. Spielauer, M. (2011). What is Social Science Microsimulation? Social Science Computer Review, 29(1), 9-20. https://doi.org/10.1177/0894439310370085.

76. Boero, R., \& Squazzoni, F. Does Empirical Embeddedness Matter? Methodological Issues on Agent-Based Models for Analytical Social Science. Journal of Artificial Societies and Social Simulation, 8(4), 6.

77. Careja, R., \& Andreß, H.-J. (2018). In search of a frame: challenges and opportunities for sampling immigrant minorities. Comparative Migration Studies, 6(1), 37. https://doi.org/10.1186/s4087 8-018-0103-5.

78. United Nations Office on Drugs and Crime. (2015). Researching hidden populations: approaches to and methodologies for generating data on trafficking in persons. New York, NY: United Nations.

79. Hammond, R. A. (2015). Appendix A: Considerations and Best Practices in Agent-Based Modeling to Inform Policy. In Wallace R., Geller A., Ogawa V.A. (Ed.), Assessing the Use of Agent-Based Models for Tobacco Regulation (pp. 161-193). Washington, D.C.: National Academies Press.

80. Clancey, W. J., Sierhuis, M., Damer, B., \& Brodsky, B. (n.d.). Cognitive modeling of social behaviors. In Cognition and Multi-Agent Interaction. Cambridge University Press. Retrieved from https ://www.cambridge.org/us/academic/subjects/psychology/cognition/cognition-and-multi-agent-inter action-cognitive-modeling-social-simulation, https:/www.cambridge.org/us/academic/subjects/ psychology/cognition

81. Windrum, P., Fagiolo, G., \& Moneta, A. (2007). Empirical Validation of Agent-Based Models: Alternatives and Prospects. Journal of Artificial Societies and Social Simulation, 10(2), 8.

Publisher's Note Springer Nature remains neutral with regard to jurisdictional claims in published maps and institutional affiliations.

\section{Affiliations}

\section{Alys McAlpine $^{1}$ (1) $\cdot$ Ligia Kiss $^{1,2}$ (C) Cathy Zimmerman ${ }^{1} \cdot$ Zaid Chalabi $^{3,4}$}

1 Gender Violence and Health Centre, Faculty of Public Health and Policy, London School of Hygiene and Tropical Medicine, London, UK

2 Institute for Global Health, Faculty of Population Health Sciences, University College London, London, UK

3 The Bartlett School of Environment, Energy and Resources, University College London, London, UK

4 Department of Public Health, Environments and Society, Faculty of Public Health and Policy, London School of Hygiene and Tropical Medicine, London, UK 